\title{
Selective Excitation of the Yellow and Blue Luminescence in n- and p-doped Gallium Nitride
}

\author{
by \\ John Snyder Colton \\ B.S. (Brigham Young University) 1994 \\ M.A. (University of California, Berkeley) 1997
}

A dissertation submitted in partial satisfaction of the requirements for the degree of

Doctor of Philosophy

in

Physics

in the

GRADUATE DIVISION

of the

UNIVERSITY OF CALIFORNIA, BERKELEY

Committee in charge:

Professor Peter Y. Yu, Chair

Professor Yuen-Ron Shen

Professor Eugene E. Haller

Fall 2000 


\section{Table of Contents}

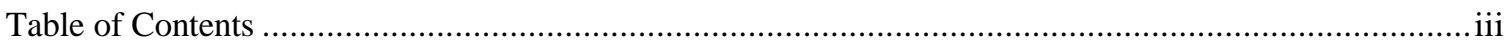

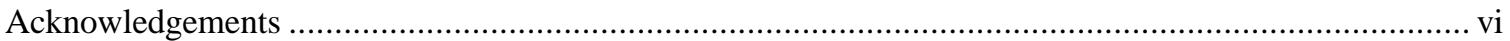

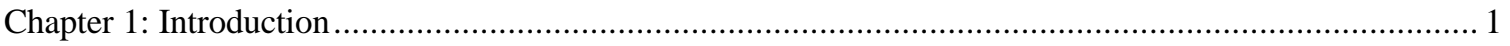

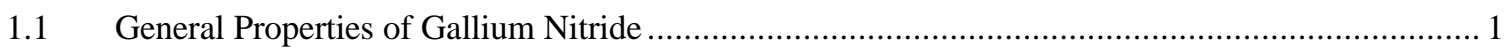

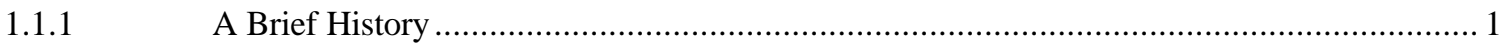

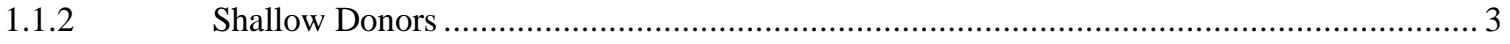

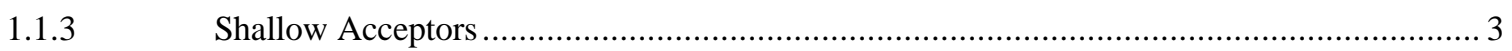

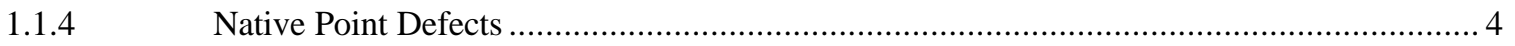

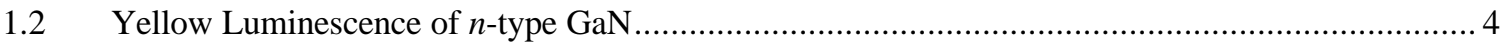

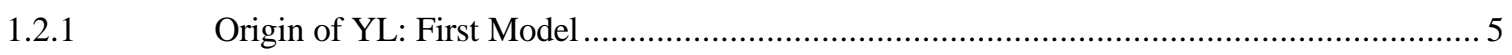

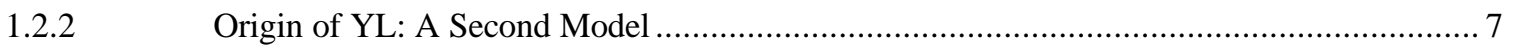

1.2.3 Origin of YL: Many Other Results and Models.......................................................... 8

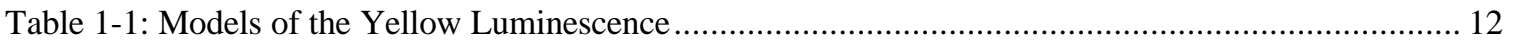

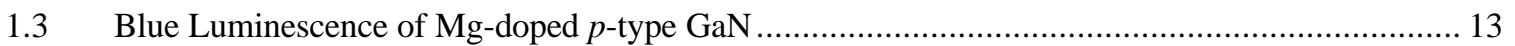

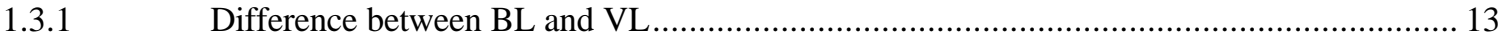

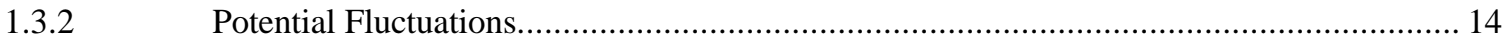

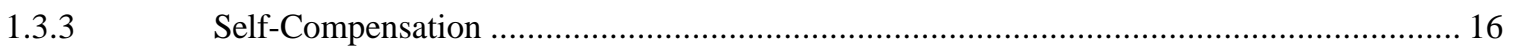

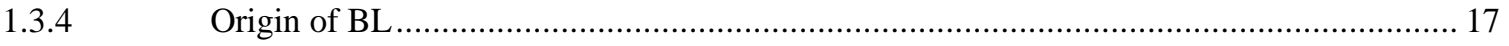

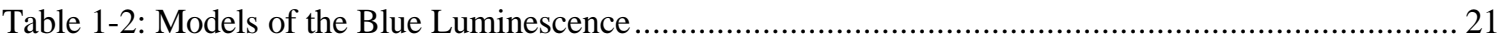

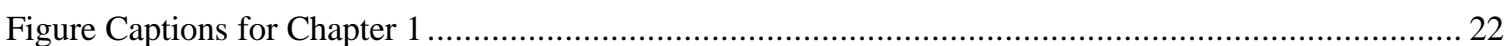

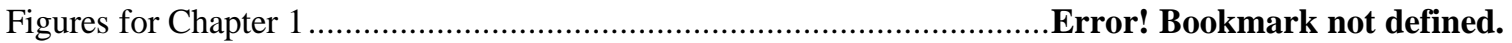

Chapter 2: Selective Excitation of the Yellow Luminescence of $n$-type GaN.......................................... 34

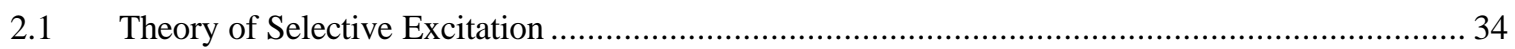

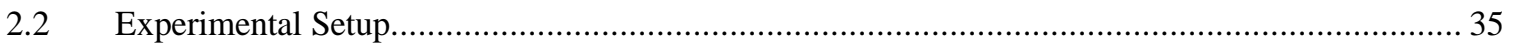

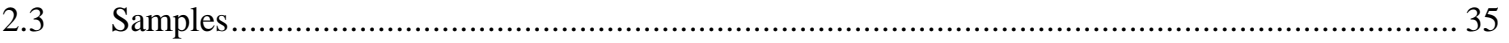

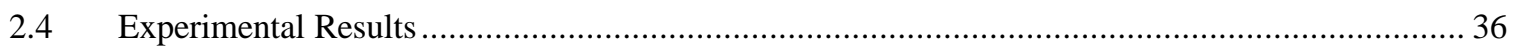




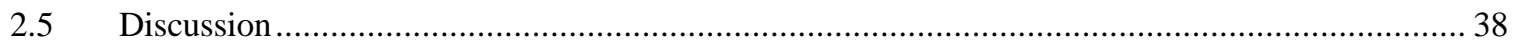

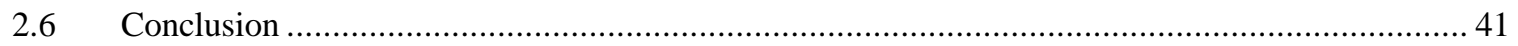

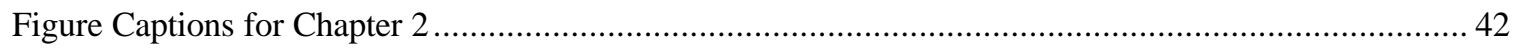

Figures for Chapter 2 .........................................................................

Chapter 3: Selective Excitation of the Blue Luminescence of Mg-doped $p$-type GaN ............................... 49

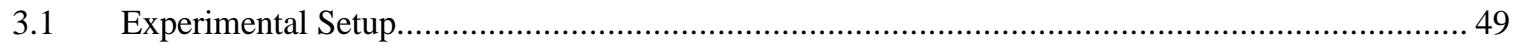

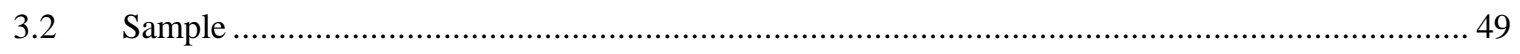

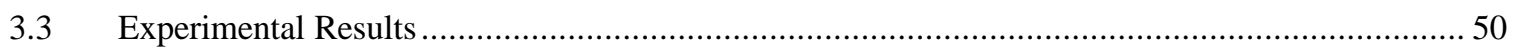

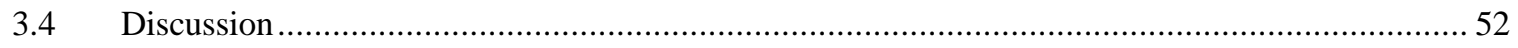

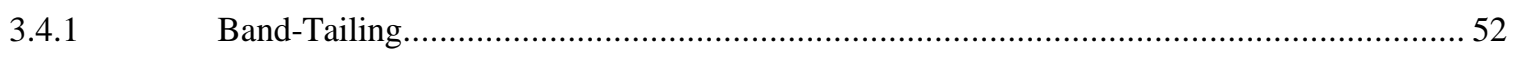

3.4.2 Frank-Condon Shift and Electron-Phonon Coupling .................................................... 54

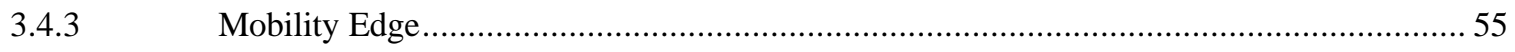

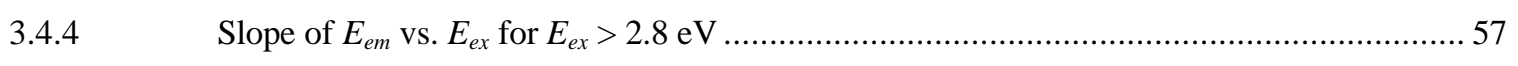

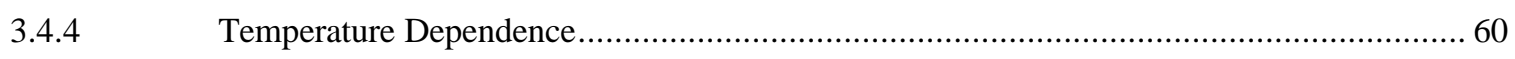

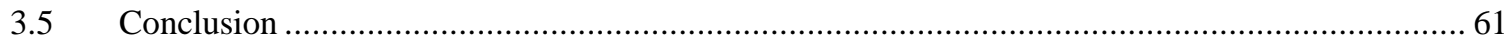

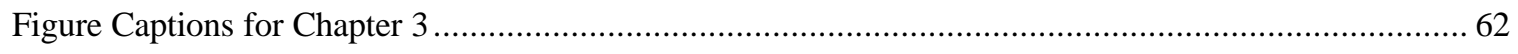

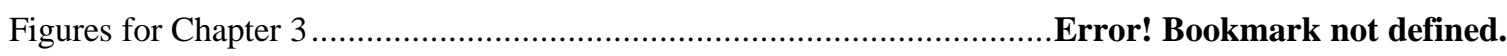

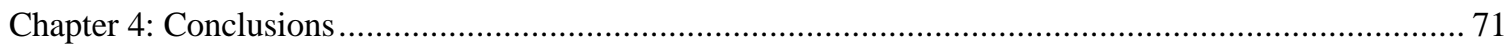

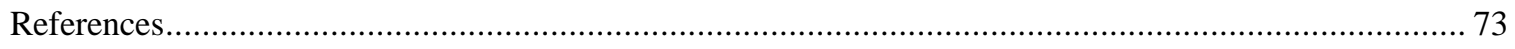




\section{Acknowledgements}

Undoubtedly the two people who have influenced me the most during the completion of my dissertation research have been my advisor, Professor Peter Y. Yu, and my wife, Pauline M. Colton. To both of them, for their great support and encouragement, I give my most sincere and heartfelt thanks. I would like to additionally thank my colleague, Dr. Kie-Leong Teo, who worked closely with me as I was beginning work in Professor Yu's laboratory. 


\section{Chapter 1: Introduction}

This dissertation is divided into four sections. Chapter 1, the introduction, deals with some properties of doped and undoped gallium nitride $(\mathrm{GaN})$, and the motivations behind my particular research. Chapter 2 deals with experiments performed to selectively excite the "yellow luminescence" of $n$-type $\mathrm{GaN}$, and includes some background information on the technique of selective excitation. Chapter 3 describes the selective excitation of the "blue luminescence" of $p$-type GaN, and includes some additional detail on the theory of highly doped semiconductors. Chapter 4 provides a summary of the conclusions arrived through the experimental research and analysis.

\subsection{General Properties of Gallium Nitride}

$\mathrm{GaN}$ is a wide band gap $\left(\mathrm{E}_{\mathrm{g}}=3.5 \mathrm{eV}\right.$ at low temperature $)$ III-V semiconductor with a direct gap, the combination of which makes it a very desirable material for a number of optical applications such as light emitting diodes (LEDs) and laser diodes. In addition, the wide range of band gap energies available through its alloys with indium $(\mathrm{GaInN})(\sim 2-3.5 \mathrm{eV})$ and aluminum $(\mathrm{AlGaN})(\sim 3.5-6 \mathrm{eV})$ make the material even more technologically promising. GaN normally crystallizes in the hexagonal wurtzite structure (see Figure 1-1), with lattice parameters of a $=3.189 \AA$ (in-plane) and c $=5.186 \AA$ (out-of-plane) [1]. In certain conditions, GaN can be made to form in the cubic zinc-blende structure. The electronic band structure and phonon dispersion have been measured and calculated by many groups, and some representative graphs for hexagonal $\mathrm{GaN}[2,3]$ are presented in Figures 1-2 and 1-3. (All of the experimental data in this work involve hexagonal GaN.)

\subsubsection{A Brief History}

A short historical description of some of the major events in the history of research on GaN is presented here. The interested reader may wish to refer to other sources (for example $[4,5,6,7]$ ) for a more complete description.

GaN crystals, in powder form, were first produced in 1932, by the reaction of ammonia gas and metallic gallium at high temperatures $\left(900-1000^{\circ} \mathrm{C}\right)$ [8]. A breakthrough in $\mathrm{GaN}$ research was when 
epitaxial GaN was first produced, via hydride vapor phase epitaxy (HVPE), in 1969 [9]. In that report, the researchers noted the high carrier concentrations $\left(n=1-5 \times 10^{19} \mathrm{~cm}^{-3}\right.$ in their samples $)$ in nominally undoped material, and the difficulty of achieving $p$-type material, both of which are important and will be discussed later in this introduction. The HVPE GaN film was grown on a sapphire substrate, which remains a popular choice today despite a $16 \%$ lattice mismatch between the two materials [4]. GaN grown on sapphire is always wurtzite, using the hexagonal sapphire as a template for growth. In the 1970s, the first light-emitting device was produced, an m-i-n structure [10]. However, the lack of a suitable lattice-matched substrate and the lack of a $p$-type dopant hindered progress for nearly two decades.

The lack of a suitable substrate bears further elaboration, since it plays a large role in many GaN characteristics. As mentioned, sapphire is one commonly used substrate, even with its large lattice mismatch. Such a large lattice mismatch is expected to produce numerous defects, especially dislocations, which typically affect luminescent efficiency very adversely. It is notable, however, that GaN seems to not suffer from this problem as much as other materials [11] (see Fig. 1-4). Another major breakthrough in GaN research occurred in 1986 when it was discovered that growing a low temperature "buffer layer" of AlN [12], or later GaN [13], in between the sapphire and the main GaN film greatly reduced formation of defects. GaN growth in both of those cases was obtained through metalorganic chemical vapor deposition (MOCVD). Some more recent efforts to reduce the amount of defects in GaN even further include (a) using substrates made from $\mathrm{SiC}$ [14], spinel $\left(\mathrm{MgAl}_{2} \mathrm{O}_{4}\right)$ [15], or other materials (which may have a more closely matched lattice constant), (b) using the technique of lateral epitaxial overgrowth [16], and (c) growing bulk (non-epitaxial) samples under high pressure [17].

Two other major breakthroughs occurred in 1989 and 1992 when the first $p$-type GaN was obtained, in Mg-doped material—first, by low energy electron beam treatment [18] and then by high temperature annealing in a hydrogen free environment [19]. The barrier to formation of p-type material was discovered to have been passivation of the $\mathrm{Mg}$ acceptors by hydrogen [20]. Starting with these breakthroughs, technological progress was rapidly made, including the production of high brightness blue LEDs [21], and culminating in the first nitride-based blue laser diode [22], both of which used GaInN as the active layer. 


\subsubsection{Shallow Donors}

As mentioned above, nominally undoped GaN has a large $n$-type background [9]. Due to the large value of $n$, the responsible donor was initially thought to be a native defect, possibly a nitrogen vacancy $\left(\mathrm{V}_{\mathrm{N}}\right)$ [23]. The dominant shallow donor in bulk samples was found to have a resonant state in the conduction band (CB), which can be seen via application of high pressure ( 20 GPa) [24]. This, combined with theoretical studies showing that $\mathrm{V}_{\mathrm{N}}$ should produce a resonant state in the $\mathrm{CB}$ [25], added credibility to $\mathrm{V}_{\mathrm{N}}$ 's identification as the donor. However, more recent experimental [26,27] and theoretical [28,29] studies have disproven the $\mathrm{V}_{\mathrm{N}}$ hypothesis, and have shown that the shallow donor is due to either unintentional silicon (replacing $\mathrm{N}, \mathrm{Si}_{\mathrm{N}}$ ) or oxygen (replacing nitrogen, $\mathrm{O}_{\mathrm{N}}$ ) donors, with only $\mathrm{O}_{\mathrm{N}}$ being found in bulk GaN samples. Thus, the oxygen donor (but not the silicon) forms the resonant state which is somewhat similar to the DX center of GaAs [30].

The electron effective mass in GaN has been measured using cyclotron resonance, and found to be $\mathrm{m}^{*}=0.22 \mathrm{~m}_{0}$ [31]. One may calculate an expected hydrogenic donor binding energy by using the static dielectric constant, $\varepsilon=9.5$, [32], and the well-known formula:

$$
\begin{aligned}
& \mathrm{E}_{\mathrm{d}}=13.6 \mathrm{eV}\left(\mathrm{m}^{*} / \mathrm{m}_{0}\right)\left(1 / \varepsilon^{2}\right) \\
& \mathrm{E}_{\mathrm{d}}=33 \mathrm{meV}
\end{aligned}
$$

This compares well with the most recent theoretical and experimental values for the $\mathrm{Si}_{\mathrm{Ga}}$ and $\mathrm{O}_{\mathrm{N}}$ shallow donors, which give ionization energies of 30.8 and $32.4 \mathrm{meV}$ for $\mathrm{Si}_{\mathrm{Ga}}$ and $\mathrm{O}_{\mathrm{N}}$ respectively [33].

Using the above values, one can also estimate the Bohr radius of the shallow donors:

$$
\begin{aligned}
& \mathrm{a}_{\mathrm{d}}=0.529 \AA\left(\mathrm{m}_{0} / \mathrm{m}^{*}\right)(\varepsilon) \\
& \mathrm{a}_{\mathrm{d}}=23 \AA
\end{aligned}
$$

\subsubsection{Shallow Acceptors}

As mentioned above, the acceptor which has received most of the recent attention is $\mathrm{Mg}$, due to the possibility of $p$-type doping, and the realization of such was indeed a major accomplishment. However, very high doping levels are required to reach a reasonable $p$-concentration; for example, typical values for $\mathrm{Mg}$ dopants of $[\mathrm{Mg}]=10^{19}-10^{20} \mathrm{~cm}^{-3}$ yield a hole concentration at room temperature of around 
$p=10^{17}-10^{18} \mathrm{~cm}^{-3}$ [34]. The Mg-acceptor binding energy from Hall effect measurements is $170-180 \mathrm{meV}$ [34]. Early optical experiments indicate a binding energy of $190 \mathrm{meV}$ [35]; however, the latest theoretical and experimental data for the binding energy of the $\mathrm{Mg}_{\mathrm{Ga}}$ acceptor indicate a value of 200-250 meV [36].

Using $200 \mathrm{meV}$ as a reasonable estimate for the hydrogenic acceptor binding energy,

$$
\mathrm{E}_{\mathrm{a}}=13.6 \mathrm{eV}\left(\mathrm{m}_{\mathrm{h}} / \mathrm{m}_{0}\right)\left(1 / \varepsilon^{2}\right)=200 \mathrm{meV}
$$

and the same value for the dielectric constant as above, one finds an effective hole mass of:

$$
\mathrm{m}_{\mathrm{h}}=\left(\mathrm{E}_{\mathrm{a}} / 13.6 \mathrm{eV}\right)\left(\varepsilon^{2}\right) \mathrm{m}_{0}=1.3 \mathrm{~m}_{0}
$$

This is fairly close to one theoretical calculation $\left(\mathrm{m}_{\mathrm{h}}=1.1 \mathrm{~m}_{0}\right)$ [37], but not particularly close to any of the wide spread of experimental values (for example, $\mathrm{m}_{\mathrm{h}}=0.54 \mathrm{~m}_{0}$ [38], $\mathrm{m}_{\mathrm{h}}=0.8 \mathrm{~m}_{0}$ [39], up to $\mathrm{m}_{\mathrm{h}}$ $\left.=2.2 \mathrm{~m}_{0}[40]\right)$. Using $\mathrm{m}_{\mathrm{h}}=1.3 \mathrm{~m}_{0}$ for the hole mass, one may estimate the hole Bohr radius:

$$
\mathrm{a}_{\mathrm{h}}=0.529 \AA\left(\mathrm{m}_{0} / \mathrm{m}_{\mathrm{h}}\right)(\varepsilon)=4 \AA
$$

\subsubsection{Native Point Defects}

Recent theoretical studies have shown that a high concentration of native point defects and defect complexes may be present in both $n$-type and $p$-type GaN. There have been numerous theoretical papers published on the subject, and many of them will be referenced later in this work. One study which bears

mentioning now, however, is the 1994 study by Neugebauer and Van de Walle [41]. Figure 1-5 reproduces the defect formation energies from their calculations, which predict that vacancies will be the dominant native defects. Specifically, the gallium vacancy $\left(\mathrm{V}_{\mathrm{Ga}}\right)$ has the lowest formation energy of any native defect in $n$-type $\mathrm{GaN}$, and the nitrogen vacancy $\left(\mathrm{V}_{\mathrm{N}}\right)$ has the lowest formation energy in $p$-type GaN.

\subsection{Yellow Luminescence of $\boldsymbol{n}$-type GaN}

$\mathrm{GaN}$ has long been known to produce defect-related luminescence. In a classic paper, Pankove and Hutchby used ion-implantation to study photoluminescence (PL) of GaN with 35 different impurities [42]. They found that 23 of the ion-implanted samples displayed a broad PL peak in the yellow, centered around $2.15 \mathrm{eV}$. Since the $2.15 \mathrm{eV}$ peak did not appear in the annealed non-implanted sample that they studied, they believed it to be implantation related. This yellow luminescence (YL) was also observed in some 
undoped samples by other groups, and in 1980 the first in-depth study trying to explain the mechanism of the YL was published by Ogino and Aoki [43].

\subsubsection{Origin of YL: First Model}

Since that 1980 study is referred to so often in the YL literature, it is worthwhile to examine some of its results and conclusions in more detail. Ogino and Aoki used two types of samples: microcrystals synthesized from a reaction between molten $\mathrm{Ga}$ and $\mathrm{NH}_{3}$, and needle-like crystals grown at the same time via sublimation and recrystallization. They found that carbon doping increases the YL, and can even cause YL to appear in samples where YL would otherwise not be present. Temperature dependent results showed that the peak position decreased with $\mathrm{T}$ (but not decreasing as much as the band-gap), and that the peak width increased with T. The YL intensity decreased with temperature in an activated fashion, with an activation energy of $860 \mathrm{meV}$. Excitation intensity dependent results showed the peak position shifting slightly to higher energies with intensity. Lastly, by comparing photoluminescence excitation (PLE) spectra of a sample with the YL to a sample without the YL, they concluded that absorption which leads to YL has a characteristic band centered around $3.19 \mathrm{eV}$.

Ogino and Aoki interpreted their results as follows: the shift of the peak position with excitation intensity led them to believe a donor-acceptor pair (DAP) transition was involved. In a DAP recombination process, the energy of the emitted phonon $(\hbar \omega)$ is given by: [44]

$$
\hbar \omega=E_{g}-E_{A}-E_{D}+e^{2} /(\varepsilon R)
$$

where $E_{g}$ is the energy gap, $E_{D}$ and $E_{A}$ are the donor and acceptor binding energies, $R$ is the distance between the donor and acceptor. The last term arises due to Coulomb interaction between the final (charged) states of the donor and acceptor. As excitation intensity increases, and more donors and acceptors are excited, the donors and acceptors involved in the recombination process will have a greater likelihood of being closer together, and a blue-shift in emission will occur. However, Ogino and Aoki note that the observed blue-shift is somewhat smaller than would be anticipated for a deep DAP transition, and postulate that the yellow band may contain contributions from conduction band (CB) to acceptor transitions as well. This could help explain their temperature results also, they noted, since one might expect a blue shift with temperature (not observed) in a transition involving a shallow donor because the electrons trapped on the 
shallow donors can be promoted to the $\mathrm{CB}$ as the temperature is raised. They pointed out that another factor to consider is the possible involvement of defect complexes; this could also help explain the smaller-thananticipated shift with excitation intensity.

The 860 meV activation energy was interpreted as an acceptor binding energy, and they proposed that the donor involved was $\mathrm{V}_{\mathrm{N}}$ (which at the time was believed to be the dominant donor, as discussed above in section 1.1.2), having a binding energy of $25 \mathrm{meV}$. Using $2.64 \mathrm{eV}$ as an estimate for the zerophonon line (since it is the midpoint between the peaks of the absorption and emission bands), that indicates the Coulomb term is about $25 \mathrm{meV}$, which corresponds to a D-A separation distance of $50 \AA$, fairly consistent with the doping levels they used.

Ogino and Aoki also employ a configuration coordinate (CC) diagram to help explain their results, which is reproduced in Figure 1-6. In the CC model, the Stokes shift between absorption and emission is explained by a lattice distortion (represented in the x-axis) between ground and excited states. Electronic transitions occur without changing the configuration (i.e., vertical on the diagram), then a subsequent lattice relaxation occurs in combination with emission of a number of phonons equal to $S$ (the Huang-Rhys factor, which in addition to representing the number of emitted phonons is also a measure of the electron-phonon coupling), thus changing the configuration until a new minimum is reached. The parameters from fitting the 4.2 K experimental data are $E_{a b}=3.19 \mathrm{eV}$ (energy for the characteristic absorption band), $E_{\text {em }}=2.13 \mathrm{eV}$ (energy of emission), $E_{0}=2.64 \mathrm{eV}$ (the zero phonon line), $\hbar \omega_{e}=40 \mathrm{meV}$ (localized phonon of excited state), $\quad \hbar \omega_{g}=41 \mathrm{meV}$ (localized phonon of ground state), $S_{a b}=13.4$ (Huang-Rhys factor of absorption process) and $S_{e m}=12.8$ (Huang-Rhys factor of emission process).

Ogino and Aoki concluded that their model fit all the experimental data fairly well, and thus the YL is from DAP recombination combined with CB-A recombination, where the donor is a shallow one, likely $\mathrm{V}_{\mathrm{N}}$, and the acceptor is a deep one, likely a complex related to carbon, such as $\mathrm{V}_{\mathrm{Ga}}-\mathrm{C}_{\mathrm{N}}$. So, to summarize, they propose the YL is mainly due to a (spatially distant) shallow donor-deep acceptor (SDDA) transition. 


\subsubsection{Origin of YL: A Second Model}

Glaser et al. proposed an alternate model for the YL in 1995, based on their optically detected magnetic resonance (ODMR) experiments [45]. They detected two resonance signals in the YL of GaN samples grown by MOCVD. One ODMR feature, with a $g$ value of 1.95 was assigned to electrons bound at hydrogenic donors, while a second feature, having a $g$ value of 1.99 was assigned to a deep donor (a deep donor rather than a deep acceptor, since acceptors typically have $g$ values above 2). No other ODMR features were seen, including any features from anticipated acceptors.

To account for the ODMR results, they proposed a two-step recombination process. (See Figure 17.) The first step, which produces the ODMR signal, is a spin dependent transition between a shallow donor and a deep donor located approximately $1 \mathrm{eV}$ below the CB. The second step, which produces the YL but no ODMR signal, is between the deep donor and a shallow acceptor. To account for the lack of an acceptor resonance signal, they proposed that the deep donor is a double-donor, with a large fraction of the doubledonors being singly occupied at the start of step 1 , due to the high Fermi level in these $n$-type films. No ODMR signals arise from the second step, because the neutral deep-donor state is doubly occupied with its spins paired off, and is therefore not paramagnetic.

Citing SIMS measurements on their samples, which indicated that the YL increased proportionally with the amount of carbon in the sample, they proposed the shallow acceptor to possibly be $\mathrm{C}_{\mathrm{N}}$. No assignment was given the deep donor, although the presence of the same deep donor in many of their samples (in all of the nominally undoped samples, and in most of the doped samples) led them to believe that it was a native defect. To summarize then, the YL in their model comes from a deep donor-shallow acceptor (DD-SA) recombination. Independent ODMR experiments have confirmed Glaser, et al.'s gvalues, and provided additional evidence for a two-stage YL model. [46]. (The most compelling additional evidence was the observation of a sharp decrease in ODMR signal when using below band-gap excitation, which implies there is a way to excite the luminescence that does not involve the spin-dependent processseemingly unexplainable unless the processes involving emission of light and magnetic-resonance effects are different.) 


\subsubsection{Origin of YL: Many Other Results and Models}

A third possible YL model was given in 1992 by Tansley and Egan [47] through comparison of an early theoretical point defect electronic state energy calculation of the amphoteric nitrogen antisite defect $\left(\mathrm{N}_{\mathrm{Ga}}\right)$ [48] to experimentally known defect levels [49]. They proposed the $\mathrm{YL}$ came from a $\mathrm{N}_{\mathrm{Ga}}$-valence band (VB) transition.

A fourth proposal, given in 1995 by Hofmann, et al. [50], was that of a radiative transition between a shallow donor and a deep donor. Essentially, two time resolved measurements led them to that proposal. First, a time-resolved measurement of the peak position showed that the YL peak did not shift with time. In a DAP transition, conversely, one expects the peak to red-shift with time, since the higher energy portions of the luminescence peak arise from spatially closer DAPs (see Eq. 1.3) whose wavefunctions have more overlap and hence a larger radiative recombination rate [51]. (Note that a very similar effect, a separation-dependent recombination rate, causes the non-exponential time decay typically seen in DAP luminescence.) Thus, they did not believe the YL was from a DAP. Secondly, they fit the experimental YL intensity's decrease with time (which was non-exponential) to the Thomas and Hopfield model [52], and found a better fit using the Bohr radius for the shallow donor than for the shallow acceptor. Thus, they believed that the luminescence did not arise from the 2-step process as described by Glaser, et al. Also, they found the YL could still be seen at excitation energies $\left(\mathrm{E}_{\mathrm{ex}}\right)$ below the band gap, $\mathrm{E}_{\mathrm{ex}}=2.8 \mathrm{eV}$, which would be unlikely in Glaser, et al.'s model. As with most groups, they believed intrinsic defects are most likely responsible, and indicated $\mathrm{V}_{\mathrm{N}}$ and or a gallium interstitial $\left(\mathrm{Ga}_{\mathrm{i}}\right)$ as possible candidates for the deep donor state.

Hoffman, et al.'s proposal corresponded well with a high pressure study by Suski, et al. [53], published shortly before the Hoffman model. The high pressure study found that the YL has a large linear shift with pressure (30 meV/GPa), which is close to the band gap shift (40 meV/GPa). The similarity in pressure dependence was cited as evidence that the YL involves electrons from either a shallow donor or conduction band, in a transition to a deep state (which could be either donor or acceptor). The linear increase of the YL peak position saturated at $2.8 \mathrm{eV}$ when a pressure of $20 \mathrm{GPa}$ was reached. Suski, et al., hypothesized that the deep state may be a $\mathrm{N}_{\mathrm{Ga}}$ antisite defect. Note that these high pressure results are also consistent with the original SD-DA model of Ogino and Aoki. 
Another important development was the application of more modern theoretical and computational methods to the YL problem. One of the most influential papers in this regard was by Neugebauer and Van de Walle in 1996 [54]. They disagreed with assignments of the deep state as a donor, since deep donors will have a very high formation energy in $n$-type material in which the YL is commonly found, and will therefore only be present in small quantities. Their calculations showed that $\mathrm{V}_{\mathrm{Ga}}$ is the only native point defect acceptor, and that it has a low formation energy in $n$-type material. Some other possibilities that had been suggested as candidates for the deep state, namely $\mathrm{V}_{\mathrm{Ga}}-\mathrm{C}_{\mathrm{N}}$ and $\mathrm{N}_{\mathrm{Ga}}$ were shown to be unlikely to form. However, a $\mathrm{V}_{\mathrm{Ga}}-\mathrm{O}_{\mathrm{N}}$ complex was found to have an even lower formation energy than the isolated $\mathrm{V}_{\mathrm{Ga}}$. Moreover, both $\mathrm{V}_{\mathrm{Ga}}$ and $\mathrm{V}_{\mathrm{Ga}}-\mathrm{O}_{\mathrm{N}}$ were found to have energy states about $1 \mathrm{eV}$ above the $\mathrm{VB}$, approximately where the DA in a SD-DA transition would be expected to lie. Lastly, they commented that involvement of a gallium vacancy or complex is consistent with some other experimental results: (1) YL is stronger near extended defects [55], where formation of point defects may be enhanced, (2) YL is suppressed under Ga-rich conditions [56], in which Ga vacancies would be less likely to form, and (3) the YL can be created via ion implantation (as seen in the early work mentioned above) [42], a destructive process which would be expected to create vacancies.

Neugebauer and Van de Walle's calculations were largely born out in a separate study by Mattila and Nieminen [29]. In fact, the case for the $\mathrm{V}_{\mathrm{Ga}} / \mathrm{V}_{\mathrm{Ga}}$-complex becomes even more compelling with the additional theoretical results. Mattila and Nieminen's calculations were able to explain the saturation of the YL blue shift in the high pressure experiments mentioned above [53], predicting a maximum YL peak of around $3 \mathrm{eV}$ due to the shallow donors ( $\mathrm{O}$ and $\mathrm{Si}$ ) transforming into deep donors. They also noted that the broad lineshape of the YL may arise from multiple factors: (1) a strong phonon coupling, as expected for a case where large lattice relaxations take place, (2) various DAP separation distances (e.g., inhomogeneous broadening from the Coulomb term), (3) variations in local environment due to trapping at dislocations, (4) possible participation of both $\mathrm{Si}$ and $\mathrm{O}$ donors, and (5) possible participation of multiple ionization levels of the deep acceptor.

A few other experiments are worth mentioning before moving on. An experiment measuring vacancies via positron annihilation indicated a linear correlation between the concentration of $\mathrm{V}_{\mathrm{Ga}}$ and the intensity of the YL [57], consistent with a SD-DA transition with $\mathrm{V}_{\mathrm{Ga}}$ or a $\mathrm{V}_{\mathrm{Ga}}$ complex as the DA. An 
experiment involving photocapacitance and deep level transient spectroscopy (DLTS) concluded that the YL was related to a deep trap around $1 \mathrm{eV}$ above the VB [58]. A Si-doping study found that the ratio of YL to band edge PL intensity increases monotonically with Si-doping and that the YL may arise from a complex involving $\mathrm{V}_{\mathrm{Ga}}$ and a Si-induced defect [59]. Two separate studies on Se-doped GaN reached different conclusions despite the same observation that YL increases with Se doping: one concluded that $\mathrm{V}_{\mathrm{Ga}}$-related deep acceptors are responsible [60], the other that $\mathrm{N}_{\mathrm{Ga}}$ is the deep state [61]. Reddy, et al., used persistent photoconductivity (PPC) experiments in cubic and hexagonal GaN (where PPC and YL were both found in the $h$-GaN but not in the $c-\mathrm{GaN}$ ) to conclude that the YL was from a SD-DD transition, with the DD possibly being a $\mathrm{N}_{\mathrm{Ga}}-\mathrm{Ga}_{\mathrm{i}}$ complex [62]. Kaufmann, et al., found the YL peak to shift with excitation intensity (similar to Ogino and Aoki), and that there was an increased shift in more highly doped samples, signs of a distant DAP transition [63]. Lastly, Shalish, et al., using contact potential difference (CPD) surface photovoltage spectroscopy, concluded that the YL arises from a CB or SD-DA transition, with a significant concentration of YL-related states coming from the sample's surface [64].

Note that there does not seem to be one single model which fits all of the results. For example, DAP models $[29,41,43,45,46,57,59,60,63]$ leave unexplained why there would be no time dependence in peak position as measured by Hofmann, et al. [50]. Currently, SD-DA models seem to have the most support (from the theoretical work [29,41], the pressure dependence [53], the positron annihilation experiment [57], etc.), but they do not explain the ODMR [45,46]. (However, it should be noted that recent observations of a GaN shallow acceptor having a $g$ value less than 2 have led Glaser to suggest that the possibility of a deep acceptor being responsible for the ODMR peak previously ascribed to a deep donor cannot be ruled out [65].)

Some of these discrepancies undoubtedly result from the poor quality of some of the samples, the growth of samples through different methods, and the inhomogeneity produced by possible involvement of extended defects such as dislocations $[55,64,66]$. Also, the possibility that more than one mechanism contributes to the YL should not be neglected. It is clear that further investigation of the YL is still warranted. 
To conclude this section, most but not all of the evidence supports a distant DAP recombination, most likely from a shallow donor to a deep acceptor. A summary table listing all relevant YL proposals is given in Table 1-1. 
Table 1-1: Models of the Yellow Luminescence

\begin{tabular}{|c|c|c|}
\hline YL Model/Observations & Authors & Type of Experiment \\
\hline $\begin{array}{l}C B / S D-D A\left(\mathrm{DA}=\mathrm{V}_{\mathrm{Ga}}-\mathrm{C}_{\mathrm{N}}\right. \\
\text { complex })\end{array}$ & Ogino \& Aoki, Dec 1980 [43] & $\begin{array}{l}\text { C-doping, temperature and } \\
\text { intensity dependence of PL }\end{array}$ \\
\hline$D D / D A-V B\left(\mathrm{DD} / \mathrm{DA}=\mathrm{N}_{\mathrm{Ga}}\right)$ & $\begin{array}{l}\text { Tansley \& Egan, May } 1992 \\
\text { [47] }\end{array}$ & $\begin{array}{l}\text { Comparison of theory with } \\
\text { experimental defect levels }\end{array}$ \\
\hline$D D-S A\left(\mathrm{SA}=\mathrm{C}_{\mathrm{N}}\right)$ & Glaser, et al., May 1995 [45] & ODMR \\
\hline $\begin{array}{l}D D / D A-V B\left(\mathrm{DD} / \mathrm{DA}=\mathrm{N}_{\mathrm{Ga}}\right), \text { or } \\
\text { possibly SD-DA }\end{array}$ & Shan, et al., Jun 1995 [67] & High pressure $\mathrm{PL}$, to $6 \mathrm{GPa}$ \\
\hline $\begin{array}{l}C B / S D-D D / D A \text { (maybe } \\
\left.\mathrm{DD}=\mathrm{N}_{\mathrm{Ga}}\right)\end{array}$ & Suski, et al., Oct 1995 [53] & High pressure $\mathrm{PL}$, to $25 \mathrm{GPa}$ \\
\hline$S D-D D\left(\mathrm{DD}=\mathrm{V}_{\mathrm{N}}\right.$ or $\left.\mathrm{Ga}_{\mathrm{i}}\right)$ & Hofmann, et al., Dec 1995 [50] & ODMR, time resolved PL \\
\hline from extended defects & Ponce, et al., Jan 1996 [55] & $\begin{array}{l}\text { Spatially resolved } \\
\text { cathodoluminescence (CL) }\end{array}$ \\
\hline $\begin{array}{l}S D-D A\left(\mathrm{SD}=\mathrm{O}_{\mathrm{N}} \text { or } \mathrm{Si}_{\mathrm{Ga}}\right. \\
\left.\mathrm{DA}=\mathrm{V}_{\mathrm{Ga}} \text { or } \mathrm{V}_{\mathrm{Ga}}-\mathrm{O}_{\mathrm{N}} \text { complex }\right)\end{array}$ & $\begin{array}{l}\text { Neugebauer \& Van de Walle, } \\
\text { Jul } 1996 \text { [54] }\end{array}$ & Theory \\
\hline$S D-D A\left(\mathrm{DA}=\mathrm{V}_{\mathrm{Ga}}\right.$ related $)$ & Yi \& Wessels, Nov 1996 [60] & Hall effect and PL of Se-doped \\
\hline$D D-S A$ & Mason, et al., 1997 [46] & ODMR \\
\hline$S D-D D / D A$ & Calleja, et al., Feb 1997 [58] & Photocapacitance \& DLTS \\
\hline $\begin{array}{l}S D-D A\left(\mathrm{SD}=\mathrm{O}_{\mathrm{N}} \text { and } \mathrm{Si}_{\mathrm{Ga}}\right. \\
\left.\mathrm{DA}=\mathrm{V}_{\mathrm{Ga}}-\mathrm{O}_{\mathrm{N}} \text { complex }\right)\end{array}$ & $\begin{array}{l}\text { Mattila \& Nieminen, Apr } 1997 \\
\text { [29] }\end{array}$ & Theory \\
\hline $\begin{array}{l}S D-D A\left(\mathrm{~V}_{\mathrm{Ga}}-\mathrm{Si} \text {-induced defect }\right. \\
\text { complex })\end{array}$ & Lee, et al., Sep 1997 [59] & PL and XRD of Si-doped \\
\hline$C B / S D-D A\left(\mathrm{DA}=\mathrm{N}_{\mathrm{Ga}}\right)$ & Chen, et al., Sept 1997 [61] & $\begin{array}{l}\text { Photoconductivity and PL of } \\
\text { Se-doped samples }\end{array}$ \\
\hline$S D-D A\left(\mathrm{~V}_{\mathrm{Ga}}\right)$ & Saarinen, et al., Oct 1997 [57] & Positron annihilation \\
\hline $\begin{array}{l}\text { "Multiple recombination } \\
\text { channels" }\end{array}$ & $\begin{array}{l}\text { Zhang \& Kuech, Mar } 1998 \\
\text { [68] }\end{array}$ & $\begin{array}{l}\text { Temperature dependence of } \\
\text { PL of } \mathrm{C} \text { - and } \mathrm{H}_{2} \text {-doped } \mathrm{GaN}\end{array}$ \\
\hline $\begin{array}{l}S D-D D\left(\mathrm{DD}=\mathrm{N}_{\mathrm{Ga}}-\mathrm{Ga}_{\mathrm{i}}\right. \\
\text { complex })\end{array}$ & Reddy, et al., Jul 1998 [62] & PPC, hex \& cubic samples \\
\hline$S D-D A$ & $\begin{array}{l}\text { Kaufmann, et al., Feb } 1999 \\
\text { [63] }\end{array}$ & $\begin{array}{l}\text { Temperature dependence, } \\
\text { power density dependence, } \\
{[\mathrm{Si}] \&[\mathrm{Mg}] \text { dependence, }} \\
\text { comparison between YL, BL, } \\
\text { and "red luminescence" }\end{array}$ \\
\hline$C B / S D-D A$ (surface state) & Shalish, et al., Apr 1999 [64] & $\begin{array}{l}\text { CPD to measure surface work } \\
\text { function }\end{array}$ \\
\hline $\begin{array}{l}\text { complexes of extended defects } \\
\text { and point defects }\end{array}$ & Li, et al., May 1999 [66] & $\begin{array}{l}\text { Spatially resolved PL of lateral } \\
\text { overgrowth }\end{array}$ \\
\hline$S D-D A\left(\mathrm{SD}=\mathrm{Si}_{\mathrm{Ga}}\right)$ & Kwon, et al., Feb 2000 [69] & $\begin{array}{l}\text { Time-resolved PL of Si-doped } \\
\text { GaN }\end{array}$ \\
\hline
\end{tabular}




\subsection{Blue Luminescence of Mg-doped $p$-type GaN}

As mentioned above in section 1.1.1, p-type GaN was first grown successfully by Mg-doping combined with post-growth electron irradiation, in 1989 [18]. The first report of photoluminescence from p-type Mg-doped GaN appeared shortly thereafter [70]. Among other things, that study reported that at least three types of luminescences appear: (1) at low temperature $(4 \mathrm{~K})$, and lower doping levels $([\mathrm{Mg}] \sim 2$ $\times 10^{19} \mathrm{~cm}^{-3}$ ), a narrow band at $3.27 \mathrm{eV}$ is seen, (2) at low temperature in higher Mg-doped samples (e.g., $\left.[\mathrm{Mg}] \sim 7 \times 10^{19} \mathrm{~cm}^{-3}\right)$ a broad PL line appears, centered around $3.0 \mathrm{eV}$, and (3) at room temperature, in similar highly-doped samples $\left(5 \times 10^{19} \mathrm{~cm}^{-3}<[\mathrm{Mg}]<2 \times 10^{20} \mathrm{~cm}^{-3}\right)$ a broad emission at $2.7 \mathrm{eV}$ was seen. It is this latter, deeper, emission that will be discussed here, and will be called the "blue luminescence" (BL) of heavily Mg-doped GaN. The broad emission seen at higher energies will be referred to as "violet luminescence" (VL). Note that the peak positions of the BL and VL often vary from the values reported in this initial study (with $\mathrm{BL} \sim 2.6 \mathrm{eV}-3.0 \mathrm{eV}$, and $\mathrm{VL} \sim 3.0 \mathrm{eV}-3.2 \mathrm{eV}$ ). The origin of the $\mathrm{BL}$ in particular has been a source of confusion to researchers, much like the origin of YL in $n$-type GaN..

\subsubsection{Difference between BL and VL}

Investigation of the BL has been complicated by the presence of these three different luminescence bands. The $3.27 \mathrm{eV}$ band has been observed by many other groups [35,71] and is not controversial, being a shallow donor - shallow acceptor transition, with a shallow substitutional impurity, $\mathrm{Mg}_{\mathrm{Ga}}$, as the acceptor. It is the relationship between the $\mathrm{VL}$ and the $\mathrm{BL}$ that has been problematical, in particular because of (a) overlap between the two bands in some PL spectra, and (b) the assumption that they had the same defect origin, in many of the initial studies.

For example, perhaps the earliest detailed study of these PL bands was by Smith, et al., in 1996 [72]. They measured the normal and time-dependent PL of two bands, one of which they called the " 3.21 $\mathrm{eV}$ peak" and the other the "2.95 eV peak". However, their temperature dependent PL spectra show the "2.95 eV peak" to appear at about $3.1 \mathrm{eV}$ at $10 \mathrm{~K}, 2.95 \mathrm{eV}$ at $150 \mathrm{~K}$, and 2.6-2.7 eV (depending on excitation intensity) at $300 \mathrm{~K}$. It seems most likely that for many temperatures they measured some combination of the BL and the VL, rather than a peak from just one origin. In contrast, another 1996 study 
observed a peak to shift continuously from $3.2 \mathrm{eV}$ to $2.87 \mathrm{eV}$ between $6 \mathrm{~K}$ and $300 \mathrm{~K}$, but assigned their peak to a combination of two peaks from two different origins [73].

As just mentioned, some experiments seem to show the VL transform into the BL with increasing temperature. The same is also true of power density experiments. For example, Oh, et al., saw a continuous shift of the peak from $2.85 \mathrm{eV}$ to $3.2 \mathrm{eV}$ as power density was changed from $0.01 \mathrm{~W} / \mathrm{cm}^{2}$ to $10 \mathrm{~W} / \mathrm{cm}^{2}$ [74].

Nevertheless, despite the confusion, many recent experiments seem to have established fairly conclusively that the BL and VL do have different origins [75, 76]. The BL is dominant over the VL at high temperatures and low power densities, and is mostly seen in highly doped samples. The VL likely arises from CB-SA transitions which are red-shifted and broadened by potential fluctuations (the effect of potential fluctuations is described in the next section). The origin of the BL, however, is still unresolved.

\subsubsection{Potential Fluctuations}

As mentioned in section 1.1.3, the concentration of holes in p-type GaN at room temperature is generally approximately two orders of magnitude less than the concentration of $\mathrm{Mg}$ impurities, even after high temperature annealing. This is partly due to the large $(\sim 200 \mathrm{meV})$ binding energy of the shallow $\mathrm{Mg}_{\mathrm{Ga}}$ acceptor, but not entirely. Other factors that could explain the lack of holes are (a) the Mg may still be partially passivated by hydrogen (as mentioned in section 1.1.1), (b) additional compensating donors may be present, or (c) both of the above. Götz, et al., in secondary ion mass spectrometry (SIMS) and Hall effect measurements of samples annealed at different temperatures found that in typical samples that display BL (which have annealing temperatures of $700-775^{\circ} \mathrm{C}$ ), the total magnesium concentration is about $6 \times 10^{19} \mathrm{~cm}^{-3}$, the shallow acceptor concentration is about $2 \times 10^{19} \mathrm{~cm}^{-3}$, the compensating donor concentration is about $3 \times 10^{18} \mathrm{~cm}^{-3}$, and (at room temperature) the hole concentration is about $10^{17} \mathrm{~cm}^{-3}$ [77]. Therefore, most likely some $\mathrm{Mg}$ is still $\mathrm{H}$-passivated, and some of it is compensated by donors. Additional evidence for compensation is provided by Eckey, et al., who concluded from the basis of Raman measurements that highly doped samples are at the same time highly compensated [78].

The possible presence of strong compensation means that the conduction and valence bands may meander due to large potential fluctuations produced by the presence of ionized donors and acceptors. Two limiting cases of high compensation are considered in the theory of Shklowskii and Efros: a lightly doped 
sample [79], and a highly doped sample [80]. In both cases, potential fluctuations become important due to the high concentration of charged particles (which generate local electric fields) relative to the electron screening. Consequently, a large modulation of electron density in space occurs. Shklowskii and Efros assumed $n$-type material for their theory, but all of their equations quoted below are applicable to $p$-type material, with appropriate substitutions.

Considering the lightly doped case first, we note that at high compensation the concentration of donors occupied by electrons is much lower than the total donor concentration. Therefore at low temperatures all electrons will be able to find donors whose energy levels are substantially lowered by the potential of charged neighboring impurities. As a consequence, the Fermi level is actually located below the isolated impurity level. Relative to the CB edge, the Fermi level is given by:

$$
\mu=-\varepsilon_{D} /\left(2^{1 / 3}(1-K)^{1 / 3}\right)
$$

where $\varepsilon_{D}$ is the normal donor binding energy and $K$ is the degree of compensation (the number of acceptors divided by the number of donors, $K=N_{A} / N_{D}$ ). This is illustrated in Figure 1.8 (a).

Potential fluctuations may be characterized by two quantities: a screening radius, $r_{s}$ and an average amplitude of potential variation, $\gamma$. The screening radius is defined as follows:

$$
r_{s}=N_{t}^{1 / 3} / n^{2 / 3}
$$

where $N_{t}$ is the combined concentration of donors and acceptors, and $n$ is the concentration of donors occupied by electrons (i.e., $n=\mathrm{N}_{\mathrm{D}}-\mathrm{N}_{\mathrm{A}}$ at low temperature). Electrons screen fluctuations that occur on a size scale greater than $r_{s}$, whereas those less than $r_{s}$ remain unscreened. The potential variation is given by:

$$
\gamma=\left(e^{2} N_{t}^{2 / 3}\right) /\left(\kappa n^{1 / 3}\right)
$$

where $e$ is the electronic charge and $\kappa$ the dielectric constant. The effect of the long-range potential variation on the energy bands is illustrated in Figure 1.8 (b). Since electrons can move into the long-range potential wells, the Fermi level is further lowered from equation (1.4) approximately by $\gamma$.

In the highly-doped case, the equations for $r_{s}$ and $\gamma$ remain the same (Eq. 1.5 and Eq 1.6), but the short-range structure of electronic states is different. Inside a well of size $r_{s}$, there are other humps and wells of shorter range. The width of these wells may be characterized by $R_{q}$ :

$$
R_{q}=a_{D}\left(N_{t} a_{D}^{3}\right)^{-1 / 9}
$$


where $a_{D}$ is the donor Bohr radius. In the case where the semiconductor is doped strongly enough to cause it to undergo an insulator-to-metallic transition, the electrons form droplets of size $R_{q}$, as shown in Figure 1.9 (a).

If the semiconductor has not undergone a metallic transition, but is doped highly enough to produce the additional short-range fluctuations, a situation such as Figure 1.9 (b) may occur. Here, a redshift of DAP emission occurs, as compared to the no-fluctuation case, as a result of spatially indirect transitions. In addition, even non-DAP emission can be modified. For example, a non-exponential PL decay and a red-shift of the PL peak with time are expected to occur, similar to the DAP case mentioned above in section 1.2.3, since each potential well resembles a single impurity center. Also, a blue-shift of the PL peak with increase in excitation intensity should occur, due to screening of the potential fluctuations from the increased number of carriers (although the effect in this case is similar to that of the DAP case mentioned in section 1.2.1, the cause is different).

The effects described above have been seen in a number of materials. For example, in highly compensated N-doped ZnSe epilayers, Bäume, et al., reported a continuous blue-shift of the PL peak with increasing excitation density; the peak transformed from a structureless broad band to several well structured DAP bands. The authors attributed the transformation to the increased screening of the potential fluctuations (i.e., a flattening out of the bands in Figure 1.9 (b)) by photoexcited carriers [81]. In GaAs implanted with $\mathrm{Mg}, \mathrm{Be}$, and $\mathrm{Cd}$, a red-shift in DAP emission with temperature was seen at low temperatures (opposite to the normal DAP temperature dependence), and was ascribed to an increase in the probability of carriers relaxing to the deeper potential wells produced by compensation [82]. A very similar result was seen in $\mathrm{N}$-doped $\mathrm{ZnSe}$ [83].

\subsubsection{Self-Compensation}

One reason for concern about compensation is the possibility that Mg-doped GaN may be "selfcompensated". This is a phenomenon whereby the presence of one type of defect (which may be shallow) creates another type of defect (which is often deep). Thus, doping with a shallow impurity may give rise to a luminescence peak much lower in energy than the band gap. This is well-known in II-VI compounds such as $\mathrm{ZnS}$, where it has been called "self-activated" (SA) luminescence $[84,85]$. In those materials, there 
is evidence that the luminescence, which is well below the band-gap, involves a transition between a shallow donor and a distant induced acceptor complex. The complex is a group II vacancy adjacent to a second shallow donor, and is sometimes called an "A-center". The SA luminescence is typically a very broad and Gaussian band, just like the YL and BL of GaN.

The possibility of the formation of such self-induced defects in $\mathrm{GaN}$ is seen in the formation energies of native defects, Fig. 1-5. As shown in this figure, a nitrogen vacancy is most likely to form when the Fermi energy is near the VB (conversely a gallium vacancy is most likely to form when the Fermi energy is near the CB). As the material is doped $p$-type, the Fermi level is lowered towards the valence band, and nitrogen vacancies become more easily formed. Since nitrogen vacancies are deep donors, they compensate the $p$-type dopants. Neugebauer and Van de Walle showed that self compensation together with a poor $\mathrm{Mg}$-solubility (due to the formation of $\mathrm{Mg}_{3} \mathrm{~N}_{2}$ precipitates), are the two most likely mechanisms limiting high hole concentrations in GaN [86] (although it should be mentioned that at least one experimental group does not believe that $\mathrm{Mg}$ solubility is an issue [74]).

Obloh, et al., have shown experimentally that self-compensation does occur in Mg-doped GaN [87]. They measured the hole density in a number of Mg-doped samples, and found that as Mg-doping was increased, the hole density reached a maximum at $[\mathrm{Mg}] \sim 2-3 \times 10^{19} \mathrm{~cm}^{-3}$, and then decreased as more $\mathrm{Mg}$ was added. Coincident with the maximum in hole density is the rapid emergence of the BL peak, which dominates over the VL in samples with $[\mathrm{Mg}]>3 \times 10^{19} \mathrm{~cm}^{-3}$. Both observations are consistent with the formation of self-compensated defects such as $\mathrm{Mg}_{\mathrm{Ga}}-\mathrm{V}_{\mathrm{N}}$ complexes, and the identification of the BL with a DAP transition between shallow $\mathrm{Mg}_{\mathrm{Ga}}$ acceptors and deep $\mathrm{Mg}_{\mathrm{Ga}}-\mathrm{V}_{\mathrm{N}}$ donors (in direct analogy with the SA luminescence).

\subsubsection{Origin of $\mathbf{B L}$}

As just mentioned in section 1.3.3, one model for the $\mathrm{BL}$ is a DD-SA transition between $\mathrm{Mg}_{\mathrm{Ga}}$ shallow acceptors and $\mathrm{Mg}_{\mathrm{Ga}}-\mathrm{V}_{\mathrm{N}}$ deep donors [87]. However, that is not the only proposed model, nor was it the first suggested. As with the YL, there have been numerous suggestions as to the origin of the deep luminescence. Also like the YL, inter-sample variations and poor sample quality are undoubtedly part of 
the difficulty in understanding the BL, and it is only compounded by the problem in distinguishing between BL and VL.

Some experiments have already been mentioned in this work. For example, Smith, et al., (who may have confused the BL and the VL) saw a nearly single exponential time dependence of BL intensity, with a decay constant of $\sim 0.3 \mathrm{~ns}$, leading them to believe it was not a DAP transition (where a nonexponential dependence would be expected, as mentioned in section 1.2.3) but rather a CB to impurity transition involving doping induced deep-level centers or complexes [72]. Oh, et al., who performed the already-mentioned power density experiments on the BL and VL, also concluded that the emission was from CB-DA, with the conduction band and deep acceptor states being severely modified by potential fluctuations [74]. Eckey, et al., similarly performed power density PL experiments [78]; however, they concluded that emission was from a DAP transition, (DD-SA in particular, to be consistent with the observation of deep donor levels via DLTS [88]), and that the characteristics were not fully explained by the potential fluctuation model.

As discussed above, in section 1.3.2, DAP emission and non-DAP emission in the presence of potential fluctuations bear a number of similarities, and thus can be difficult to separate. In fact, distinguishing between the two was precisely one of the main topics addressed by Reshchikov, et al.'s 1999 paper [76]. In that work, which reported the excitation density dependence and temperature dependence of the PL for a number of differently doped samples, the authors concluded that potential fluctuations alone did explain the features of the VL (which is now accepted as a CB-SA transition red-shifted and broadened by the fluctuations, as mentioned in section 1.3.1), but did not explain all the features of the BL. Thus, they concluded that the BL must be due to DAPs (with potential fluctuations perhaps playing a secondary role). The main strength of their argument was that a red-shift of peak position vs. temperature was seen at high power density. The red-shift was not due to potential fluctuations, since it was reduced when the power density was reduced. One would expect a larger red shift at reduced power density if the shift was a result of potential fluctuation effects, since screening of fluctuations would be reduced due to decreased screening; hence this observation could only be explained by a standard DAP emission. Conversely, the red-shift of the VL did increase as power density was reduced, consistent with the potential fluctuation 
model. Based on the activation energy which they measured for the BL (300-400 meV), Reshchikov, et al., believed that the DAP transition responsible for the BL was DD-SA.

Seitz, et al., performed time-resolved PL and found that the overall BL peak shifted to lower energies with time, as would be expected for a DAP transition. However, they believed that the shift was not a progressive change in the peak position with time, but rather the result of a redistribution in intensity among four overlapping bands, each of which had a different exponential time decay and a peak position which did not shift with time [89]. This results disagreed with another time-dependent study, which found a non-exponential decay of the BL, and concluded that the BL was due to a DAP transition [69].

Kaufmann, et al., did some extensive studies [75,90], from which they concluded that the BL occurs simultaneously with the VL for $[\mathrm{Mg}] \sim 1-2 \times 10^{19} \mathrm{~cm}^{-3}$, and that BL is seen alone for $[\mathrm{Mg}]>2 \times$ $10^{19} \mathrm{~cm}^{-3}$. Based largely on comparisons with the SA luminescence in II-VI compounds (see section 1.3.3 and reference 87), these authors supported the spatially separate DD-SA recombination model, with the deep donor attributed to a self-compensated $\mathrm{Mg}_{\mathrm{Ga}}-\mathrm{V}_{\mathrm{N}}$ complex. This viewpoint has also been reinforced by theoretical work. In particular, Park and Chadi's theory demonstrated that various charge states of $\mathrm{V}_{\mathrm{N}}$ and $\mathrm{Mg}-\mathrm{V}_{\mathrm{N}}$ deep acceptors have formation energies as well as mid-gap energy levels which are consistent with the properties of the BL [91]. Their results are also in agreement with the theory of Van de Walle, et al. [28], although another theoretical group has proposed that the BL is caused by a DD-VB transition, with the deep donor being a defect complex composed of a $\mathrm{Mg}$ interstitial $\left(\mathrm{Mg}_{\mathrm{i}}\right)$ and a nitrogen vacancy whose formation is enhanced by hydrogenation [92].

Some recent experimental studies have provided further support for the DD-SA argument. Shahedipour, et al., based on a study of the PL and the hole concentration dependence on the annealing temperature, concluded that a deep $\mathrm{V}_{\mathrm{N}}-\mathrm{H}$ donor complex is responsible for the BL [93]. Suski, et al., have performed high-pressure experiments which indicate that the pressure dependence of the BL peak position is much smaller than that of the band-gap, and is thus unlikely to be associated with a CB or SD transition [94]. However, the ODMR studies by Glaser, et al., suggest that most likely no deep donor is involved [45], and the similarity between ODMR of the BL and the VL have even led Glaser to speculate that the BL may be a SD-SA transition, severely a red-shifted by potential fluctuations (even more severely than the VL) [95]. 
So, to summarize, the deep defect (or defects) responsible for the BL is still very much debatable (assuming that a deep defect is indeed involved). Moreover, there has been no consensus as to whether the deep defect is a deep donor or a deep acceptor. Also in dispute is whether the transition is from DAPs, and whether potential fluctuations play a large role in its properties. A summary table listing all proposed models for the BL is given in Table 1-2. As with the YL, more research is warranted. 
Table 1-2: Models of the Blue Luminescence

\begin{tabular}{|c|c|c|}
\hline BL Model/Observations & Authors & Type of Experiment \\
\hline $\begin{array}{l}C B-D D / D A(\mathrm{DD} / \mathrm{DA}=\mathrm{Mg}- \\
\text { related center, possibly a } \\
\text { complex) }\end{array}$ & Smith et al., Apr 1996 [72] & Time-resolved PL \\
\hline deep Mg complex & Myoung, et al., Oct 1996 [73] & $\begin{array}{l}\text { Temperature dependence of } \\
\text { PL and CL }\end{array}$ \\
\hline $\begin{array}{l}4 \text { different non-DAP } \\
\text { emissions }\end{array}$ & Seitz, et al., 1997 [89] & Time resolved PL \\
\hline $\begin{array}{l}C B / S D-D A\left(\mathrm{DA}=\mathrm{V}_{\mathrm{N}} \text { or } \mathrm{Mg}-\right. \\
\mathrm{V}_{\mathrm{N}} \text { complex }\end{array}$ & Park \& Chadi, May 1997 [91] & Theory \\
\hline $\begin{array}{l}C B / S D-D A\left(\mathrm{DA}=\mathrm{V}_{\mathrm{N}} \text { or } \mathrm{V}_{\mathrm{N}}\right. \\
\text { complex) }\end{array}$ & Van de Walle, et al., 1997 [28] & Theory \\
\hline$C B-D A$ & Oh, et al., Jan 1998 [74] & $\begin{array}{l}\text { Power density dependence of } \\
\text { PL }\end{array}$ \\
\hline$C B-D A\left(\mathrm{DA}=\mathrm{Mg}_{\mathrm{Ga}}\right)$ & $\begin{array}{l}\text { Viswanath, et al., Feb } 1998 \\
\text { [71] }\end{array}$ & $\begin{array}{l}\text { Temperature dependence of } \\
\text { PL for various doped samples }\end{array}$ \\
\hline $\begin{array}{l}D D-S A\left(\mathrm{DD}=\mathrm{Mg}_{\mathrm{Ga}}-\mathrm{V}_{\mathrm{N}}\right. \\
\left.\text { complex, } \mathrm{SA}=\mathrm{Mg}_{\mathrm{Ga}}\right)\end{array}$ & $\begin{array}{l}\text { Kaufmann, et al., Mar } 1998 \\
\text { [75] }\end{array}$ & $\begin{array}{l}\text { Power density dependence of } \\
\text { PL for various doped samples }\end{array}$ \\
\hline $\begin{array}{l}D D-S A \mathrm{DD}=\mathrm{Mg}_{\mathrm{Ga}}-\mathrm{V}_{\mathrm{N}} \\
\left.\text { complex, } \mathrm{SA}=\mathrm{Mg}_{\mathrm{Ga}}\right)\end{array}$ & Obloh, et al., 1998 [87] & $\begin{array}{l}\text { Power density dependence of } \\
\text { PL, measurement of hole } \\
\text { density vs. Mg concentration } \\
\text { for numerous samples }\end{array}$ \\
\hline $\begin{array}{l}D D-S A\left(\mathrm{DD}=\mathrm{Mg}_{\mathrm{Ga}}-\mathrm{V}_{\mathrm{N}}\right. \\
\left.\text { complex, } \mathrm{SA}=\mathrm{Mg}_{\mathrm{Ga}}\right)\end{array}$ & $\begin{array}{l}\text { Kaufmann, et al., Feb } 1999 \\
\text { [90] }\end{array}$ & $\begin{array}{l}\text { Temperature dependence, } \\
\text { power density dependence, } \\
{[\mathrm{Si}] \&[\mathrm{Mg}] \text { dependence, }} \\
\text { comparison between YL, BL, } \\
\text { and "red luminescence" }\end{array}$ \\
\hline$S D-D A(\mathrm{DA}=\mathrm{Mg}$-related $)$ & Sheu, et al., Oct 1998 [96] & $\begin{array}{l}\mathrm{Mg}] \text { dependence, power } \\
\text { density dependence, } \\
\text { temperature dependence }\end{array}$ \\
\hline$D D-S A$ & Eckey, et al., Nov 1998 [78] & Power density dependence \\
\hline$D D-S A$ & $\begin{array}{l}\text { Reshchikov, et al., May } 1999 \\
\text { [76] }\end{array}$ & $\begin{array}{l}\text { Many samples, power density } \\
\text { dependence, temperature } \\
\text { dependence }\end{array}$ \\
\hline $\begin{array}{l}D D-V B\left(\mathrm{DD}=\mathrm{Mg}_{\mathrm{i}}-\mathrm{V}_{\mathrm{N}}\right. \\
\text { complex, possibly } \\
\text { hydrogenated) }\end{array}$ & Lee \& Chang, Feb 1999 [92] & Theory \\
\hline $\begin{array}{l}D D-S A\left(\mathrm{DD}=\mathrm{Mg}_{\mathrm{Ga}}-\mathrm{V}_{\mathrm{N}}\right. \\
\left.\text { complex, } \mathrm{SA}=\mathrm{Mg}_{\mathrm{Ga}}\right)\end{array}$ & Kwon, et al., Feb 2000 [69] & Time-resolved PL \\
\hline $\begin{array}{l}\text { probably no } D D \text { involved, } \\
\text { possible } S D-S A\end{array}$ & Glaser, 2000 [95] & ODMR \\
\hline$D D-S A$ & Suski, et al., 2000 [94] & Pressure dependence of BL \\
\hline $\begin{array}{l}D D-S A\left(\mathrm{DD}=\left(\mathrm{V}_{\mathrm{N}}-\mathrm{H}\right)^{+2}\right. \\
\text { complex })\end{array}$ & $\begin{array}{l}\text { Shahedipour } \\
\text { \& Wessels, May } 2000 \text { [93] }\end{array}$ & $\begin{array}{l}\text { PL of differently doped } \\
\text { samples }\end{array}$ \\
\hline
\end{tabular}




\section{Figure Captions for Chapter 1}

Fig. 1-1 Diagram of the wurtzite crystal structure. Reproduced from reference 97.

Fig. 1-2 Electronic band structure of GaN, calculated via the empirical pseudopotential method. Reproduced from reference 2 .

Fig. 1-3 Phonon dispersion of $\mathrm{GaN}$, calculated via a rigid-ion model fit of experimental neutron scattering data. Reproduced from reference 3.

Fig. 1-4 Dependence of LED efficiency on dislocation density for devices made with a wide range of III-V materials, reproduced from reference 11. Etch pit density (x-axis) refers to the density of dislocations measured at the surface. The GaN samples measured in reference 11 had a dislocation density of $2-10 \times$ $10^{10} \mathrm{~cm}^{-2}$, yet still displayed efficient luminescence. The numbers next to the material names indicate references used in the original paper, from which normalized data were obtained by extrapolating to low dislocation densities.

Fig. 1-5 Defect formation energies as a function of the Fermi level for all native defects in GaN under nitrogen-rich conditions. $\mathrm{E}_{\mathrm{F}}=0$ corresponds to the top of the valence band. Reproduced from reference 41 .

Fig. 1-6 Configuration coordinate model of the YL, which explains the relation between the excitation and emission bands according to the model of Ogino and Aoki. The parameters from fitting the $4.2 \mathrm{~K}$ experimental data are $\mathrm{E}_{\mathrm{ab}}=3.19 \mathrm{eV}, \mathrm{E}_{\mathrm{em}}=2.13 \mathrm{eV}, \mathrm{E}_{0}=2.64 \mathrm{eV}, \hbar \omega_{\mathrm{e}}=40 \mathrm{meV}, \quad \hbar \omega_{\mathrm{g}}=41 \mathrm{meV}, \mathrm{S}_{\mathrm{ab}}=13.4$ and $\mathrm{S}_{\mathrm{em}}=12.8$ (see text for description of those variables). Reproduced from reference 43 .

Fig. 1-7 Model proposed to explain YL, based on ODMR results. YL proceeds through the arrows on the right, with step 1 being non-radiative, and step 2 being radiative and having an energy of $2.2 \mathrm{eV}$. Step 1 leads to magnetic resonance signals (see text) whereas step 2 does not. Reproduced from reference 45. 
Fig. 1-8 The impurity band of lightly doped and highly compensated semiconductors. (a) Energy diagram neglecting the long-range potential fluctuations. The solid line shows the conduction band edge, the short dashes represent donor levels ( $E_{0}$ is the average donor ionization energy), and the black dots represent electrons occupying these levels. The density of donor states is shown on the right, with occupied states shaded. Note that the Fermi level $(\mu)$ is lowered from the peak in the density of states. (b) Energy diagram including the long-rage potential fluctuations. Meandering lines, solid and dashed, represent the conduction band edge and the local peak of the donor level density of states respectively. The straight solid and dashed lines correspond to their unperturbed energies. The local density of donor states is indicated for one point in space by $g_{\text {loc }}$. The approximate peak-to-peak distance of the fluctuations is $r_{s}$, and the approximate amplitude is $\gamma$ (as discussed in the text). Reproduced with modifications from reference 79 .

Fig. 1.9 (a) Diagram of the conduction band edge of a highly doped, highly compensated semiconductor. The upper solid straight line represents the $\mathrm{CB}$ edge in the absence of impurity potential. $R_{q}$ and $r_{s}$ are the short- and long-range potential fluctuation distances, respectively. The lower solid straight line shows the Fermi level. Regions occupied by electrons (electronic droplets) are shaded. The symbols $\varepsilon_{l}$ and $V_{c}$ are not discussed in this work. The valence band edge is expected to have similar fluctuations. Reproduced from reference 80. (b) Schematic representation of red-shifted, spatially indirect DAP transitions which arise due to strong potential fluctuations. Reproduced from reference 81 . 


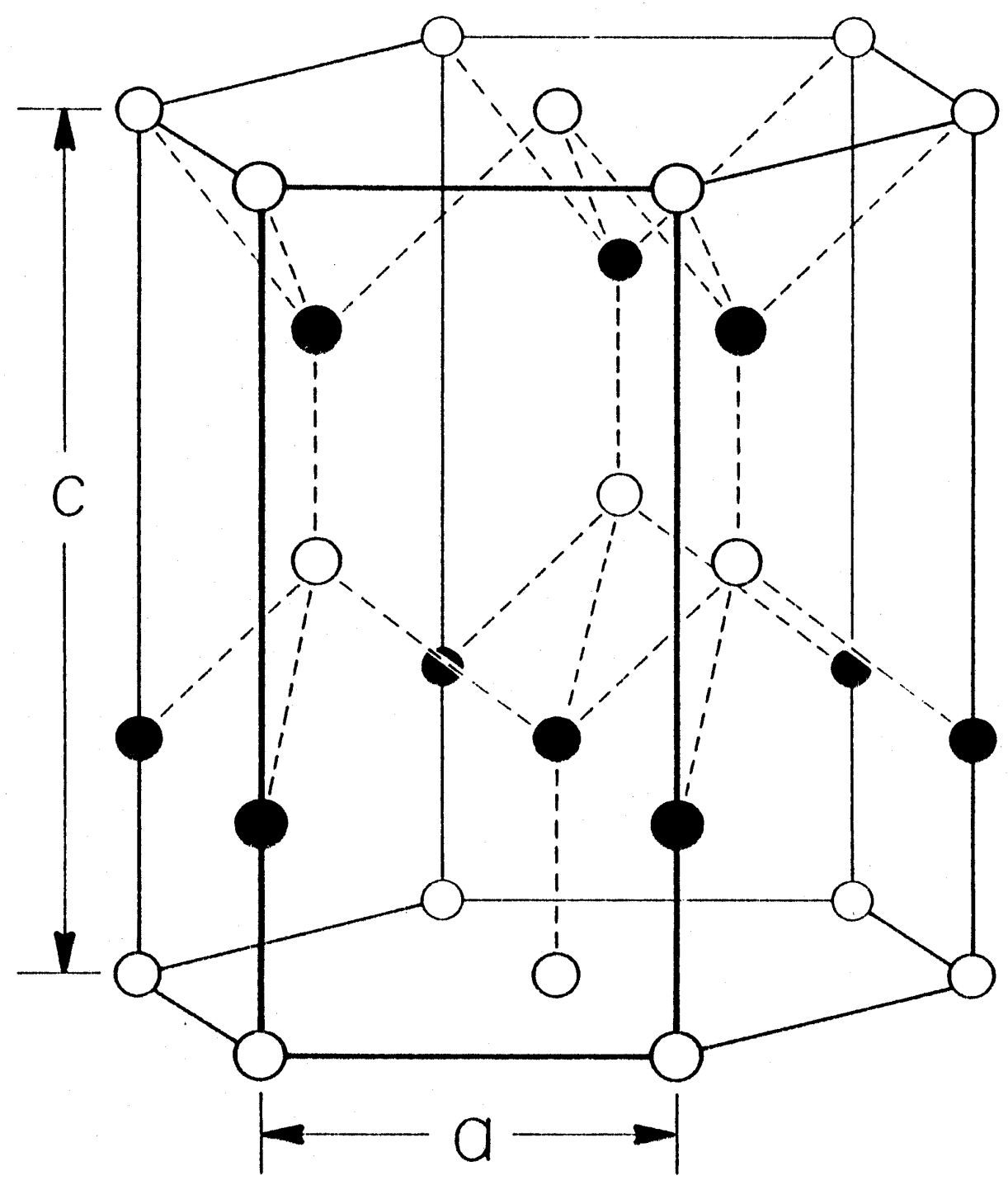

Figure 1-1 


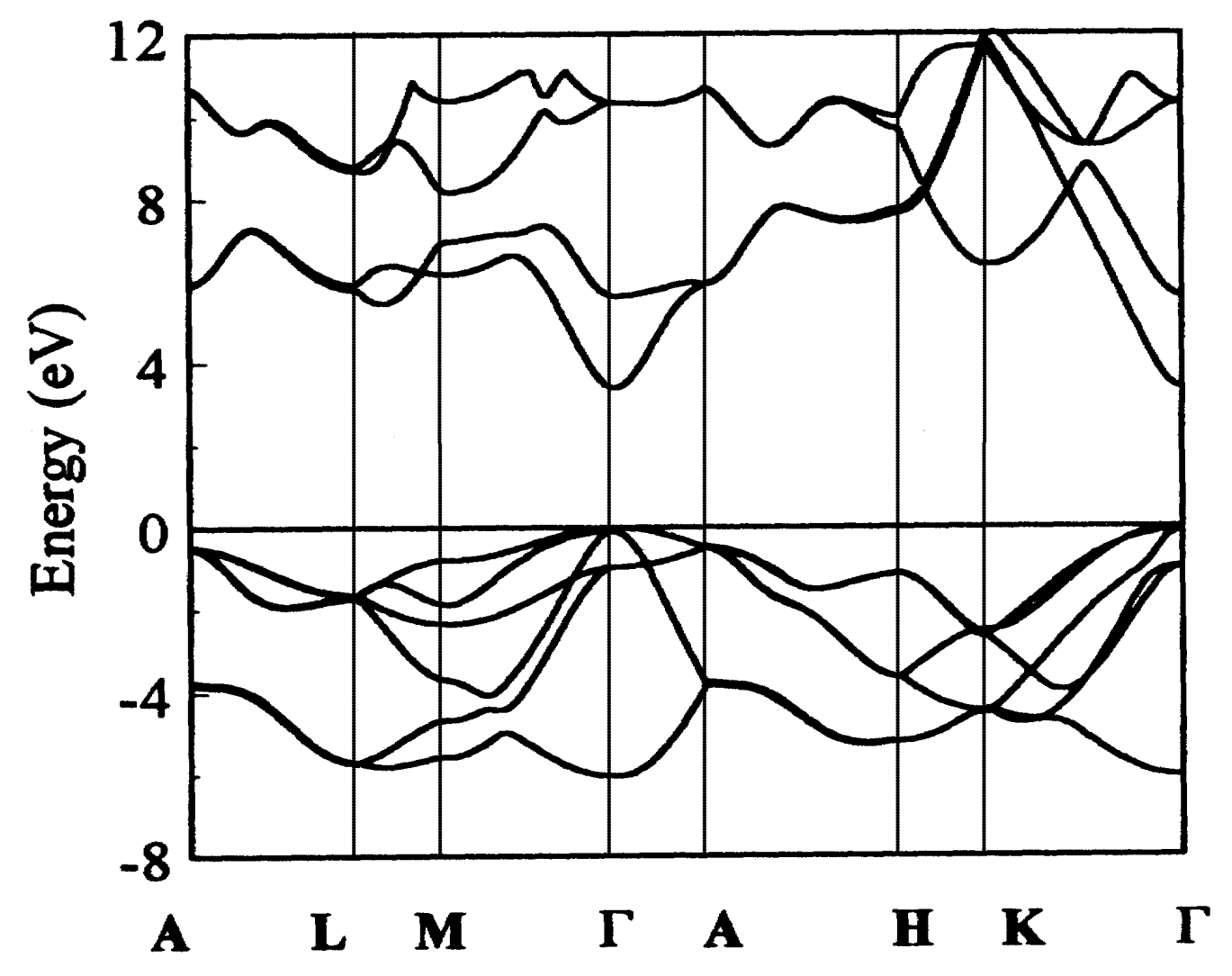

Figure 1-2 


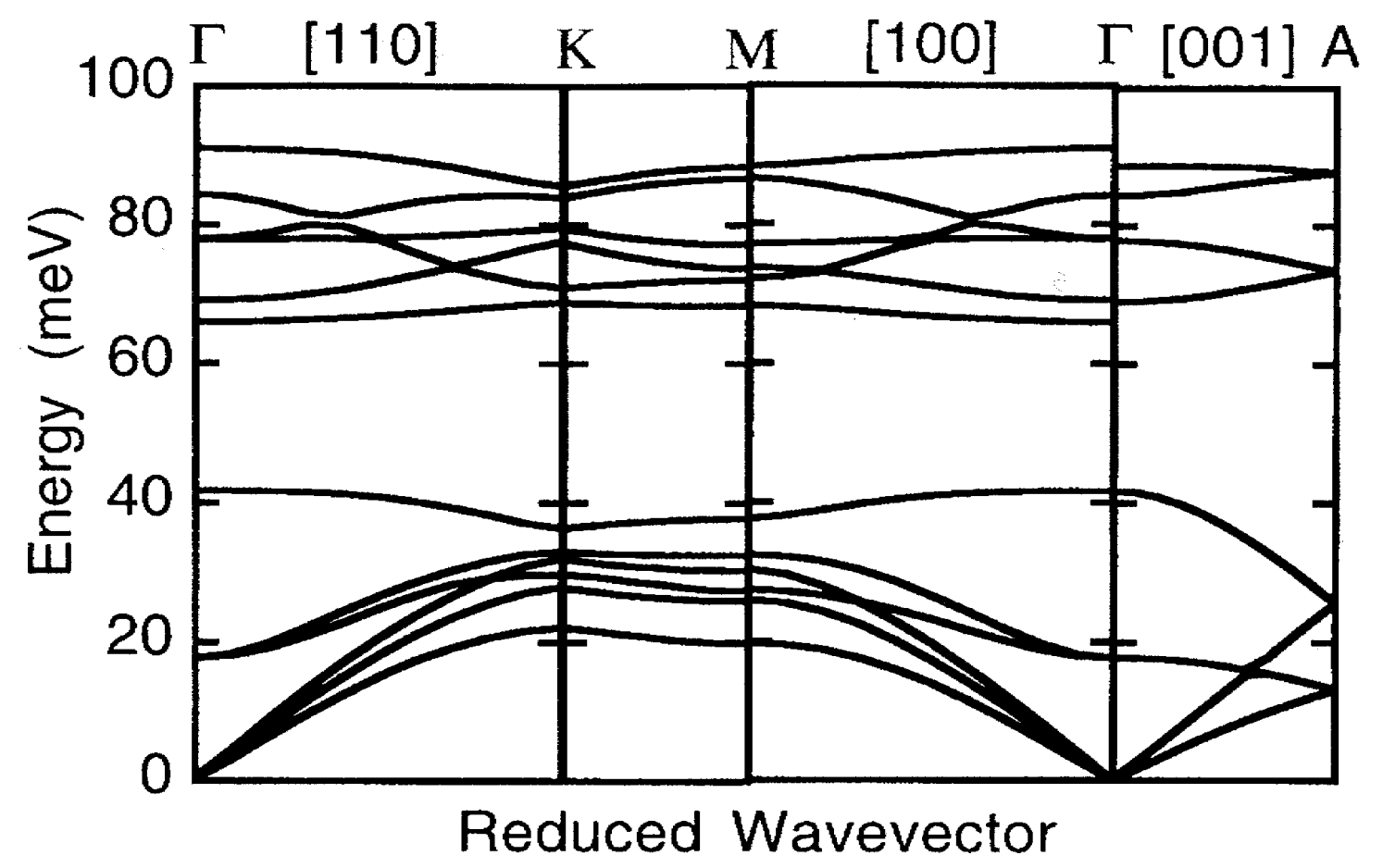

Figure 1-3 


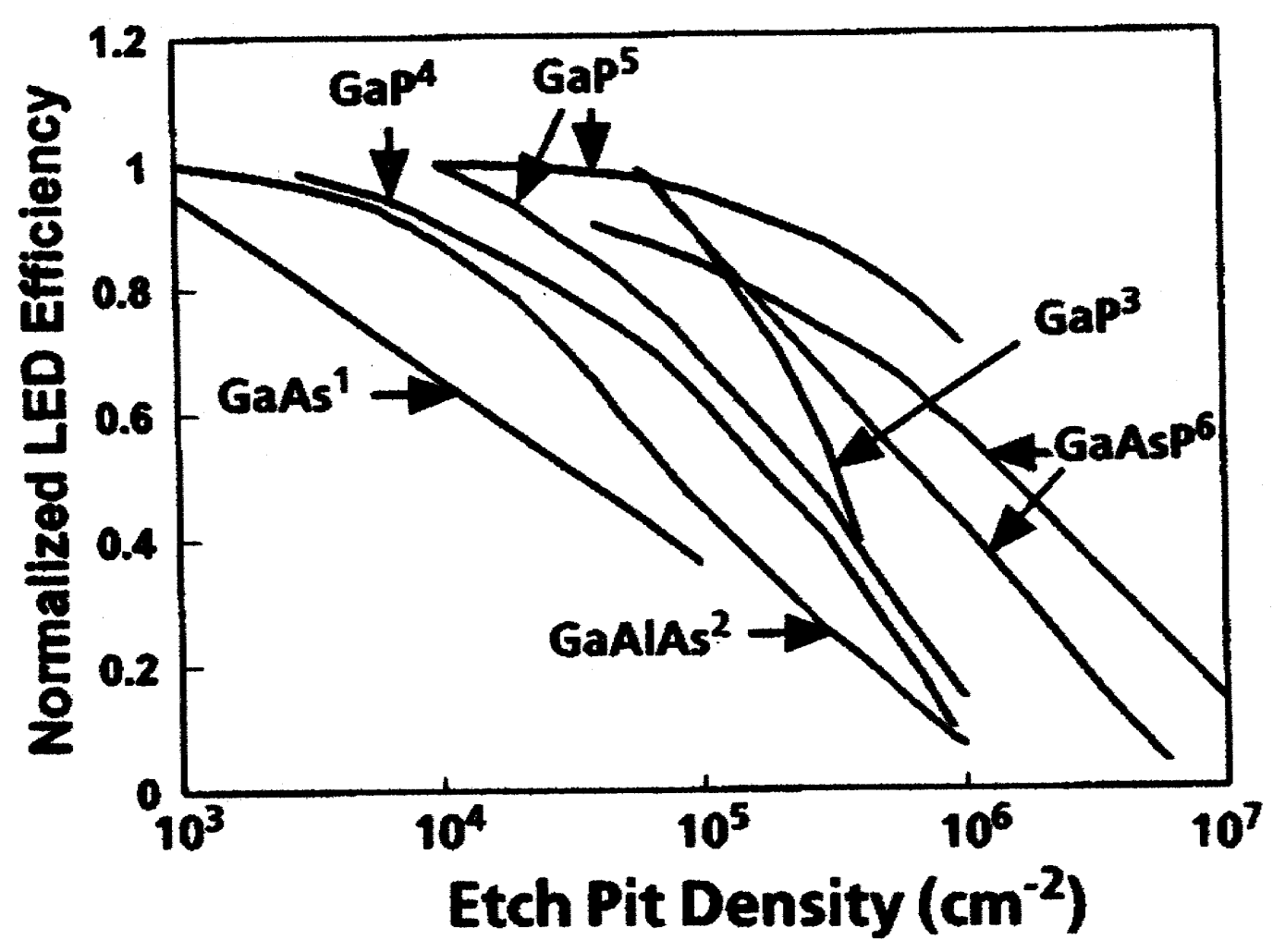

Figure 1-4 


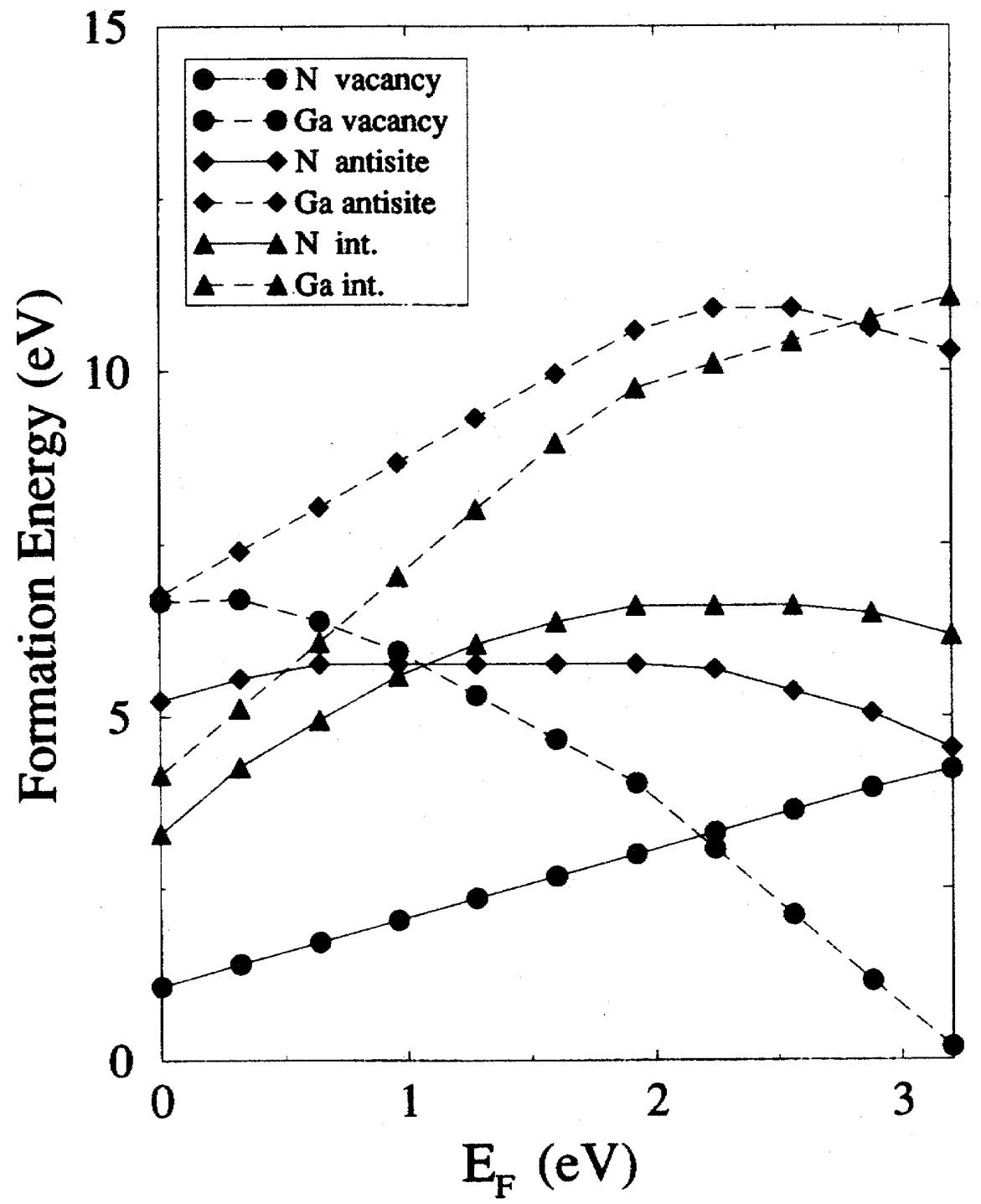

Figure 1-5 


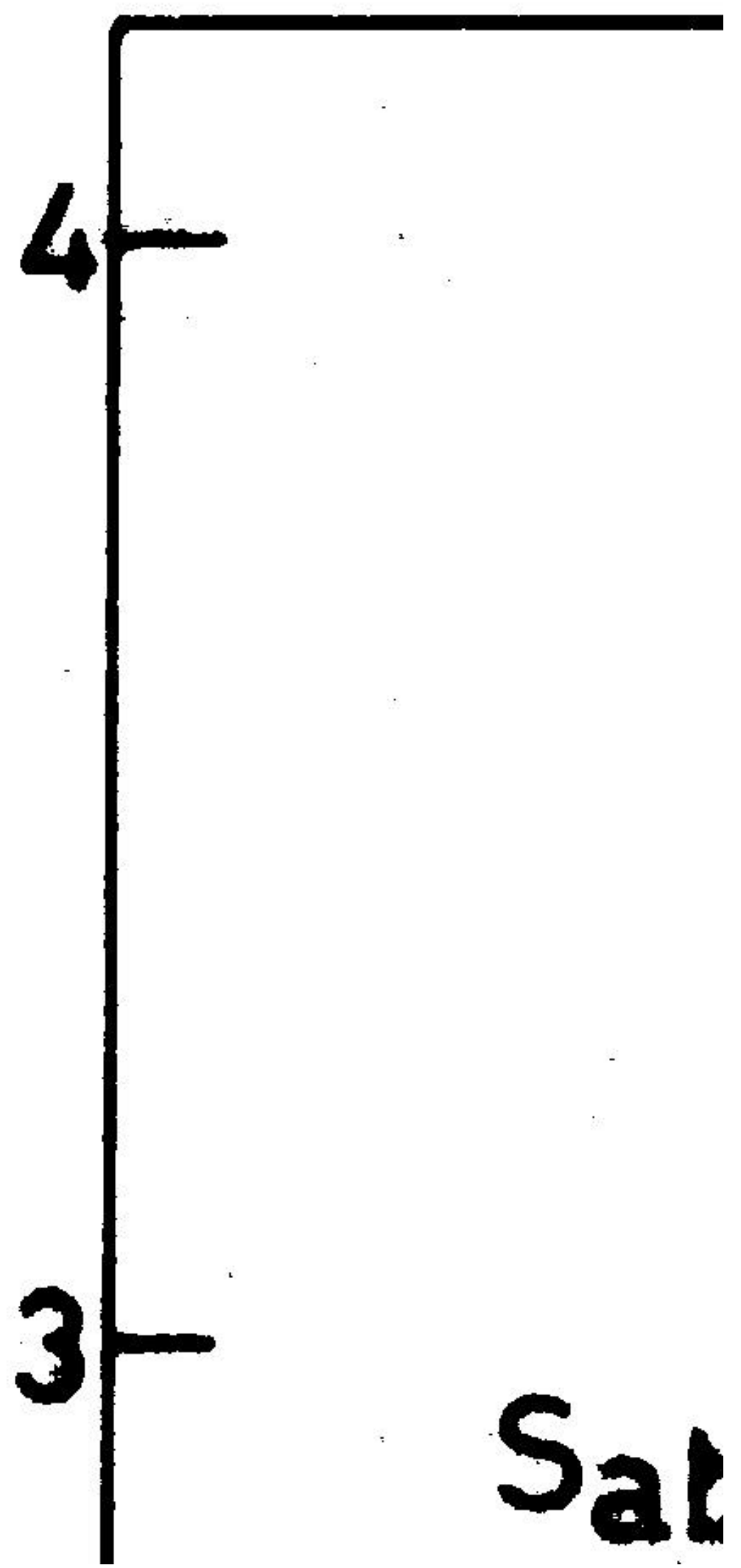


Figure 1-6 


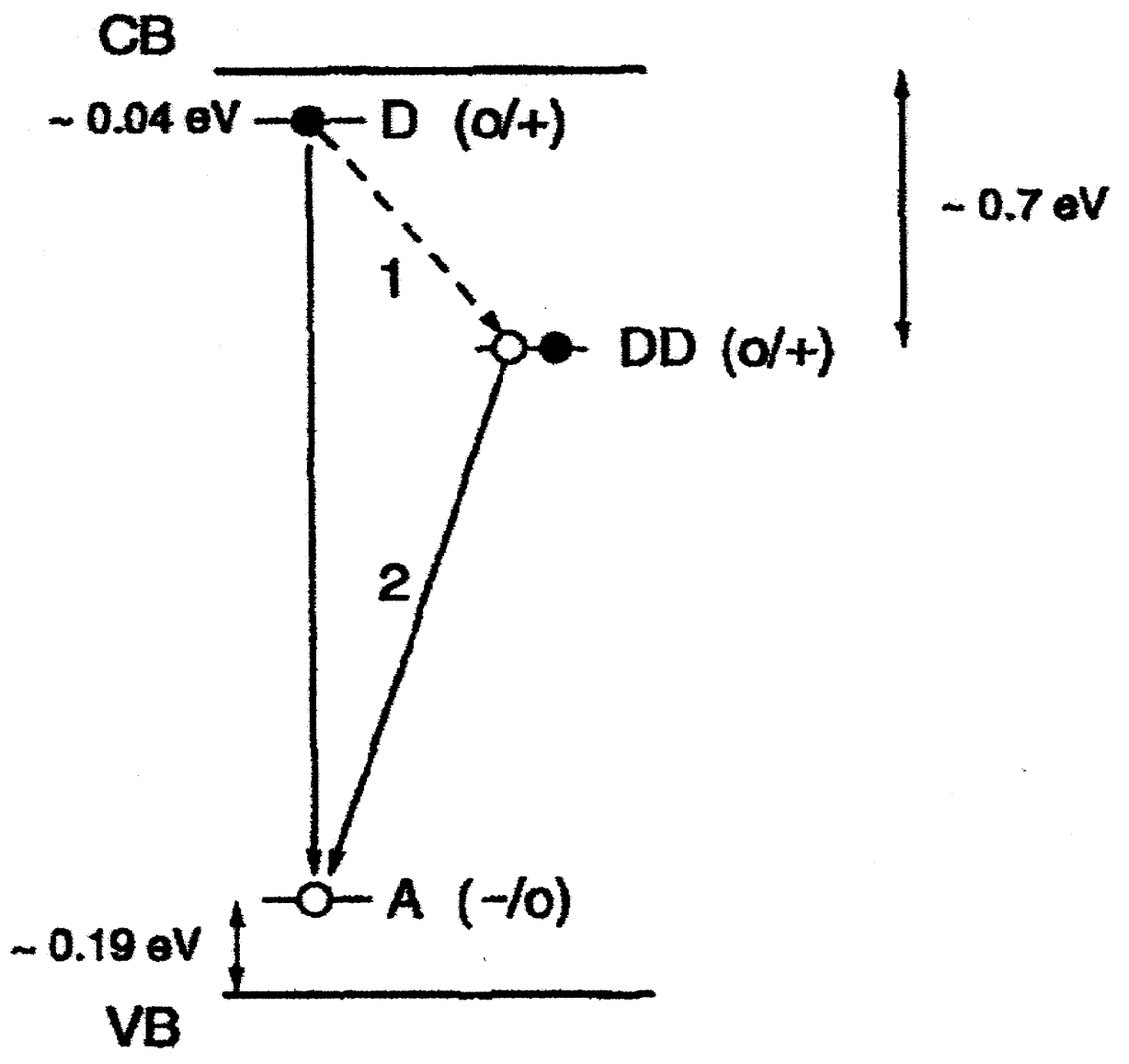

Figure 1-7 
(a)

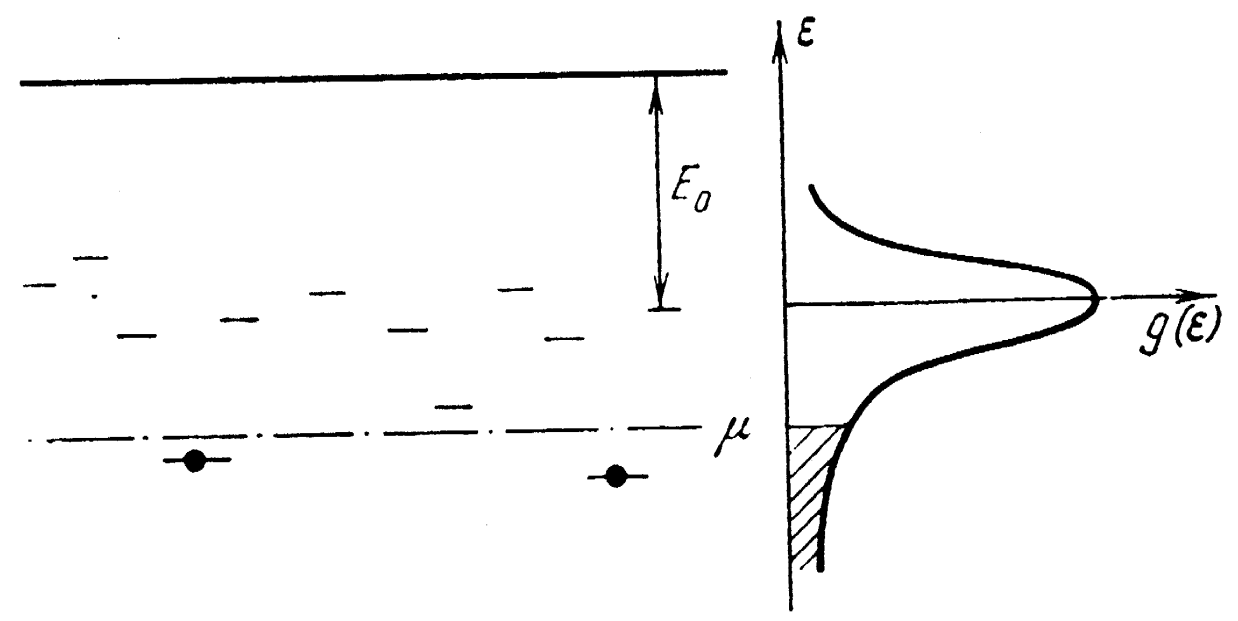

(b)

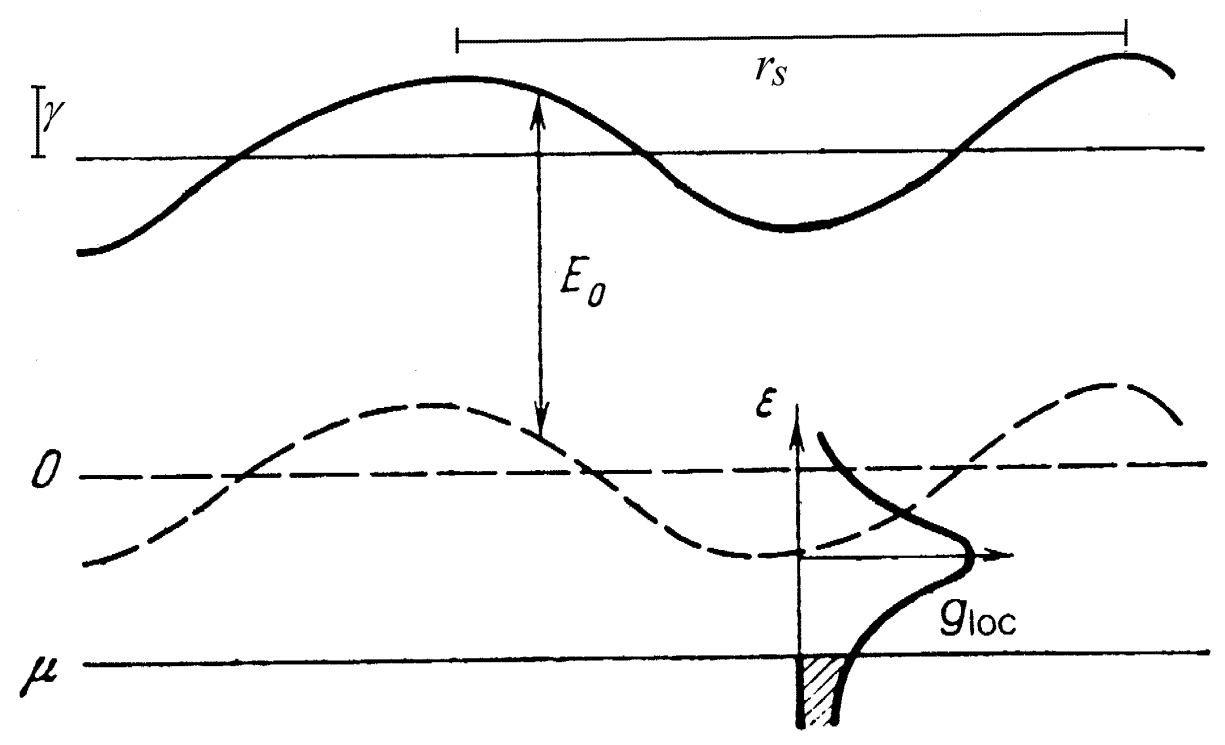

Figure 1-8 


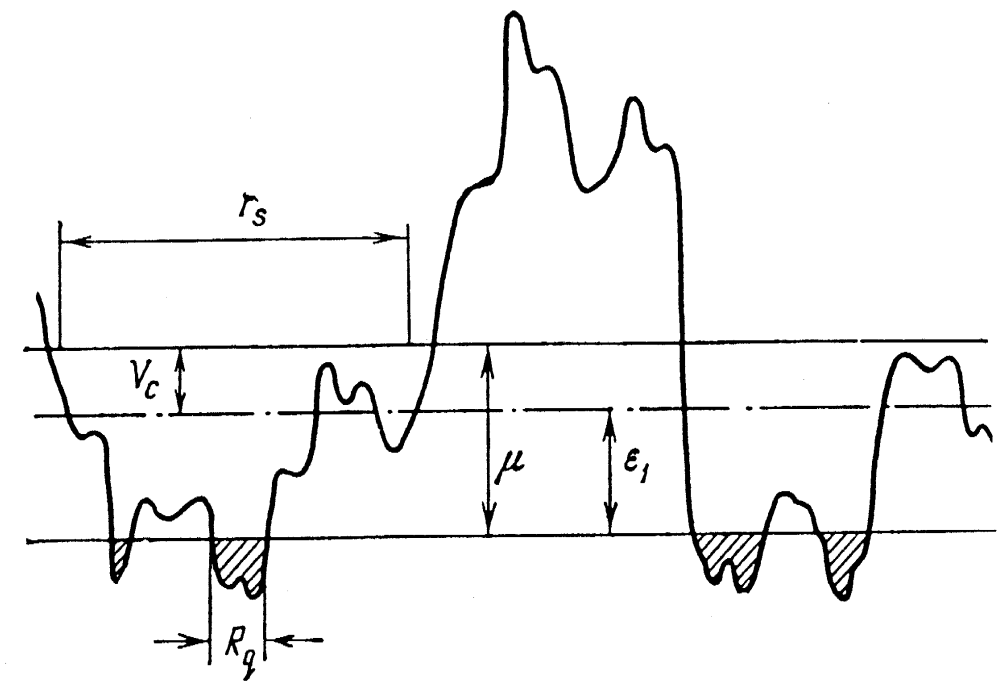

(b)

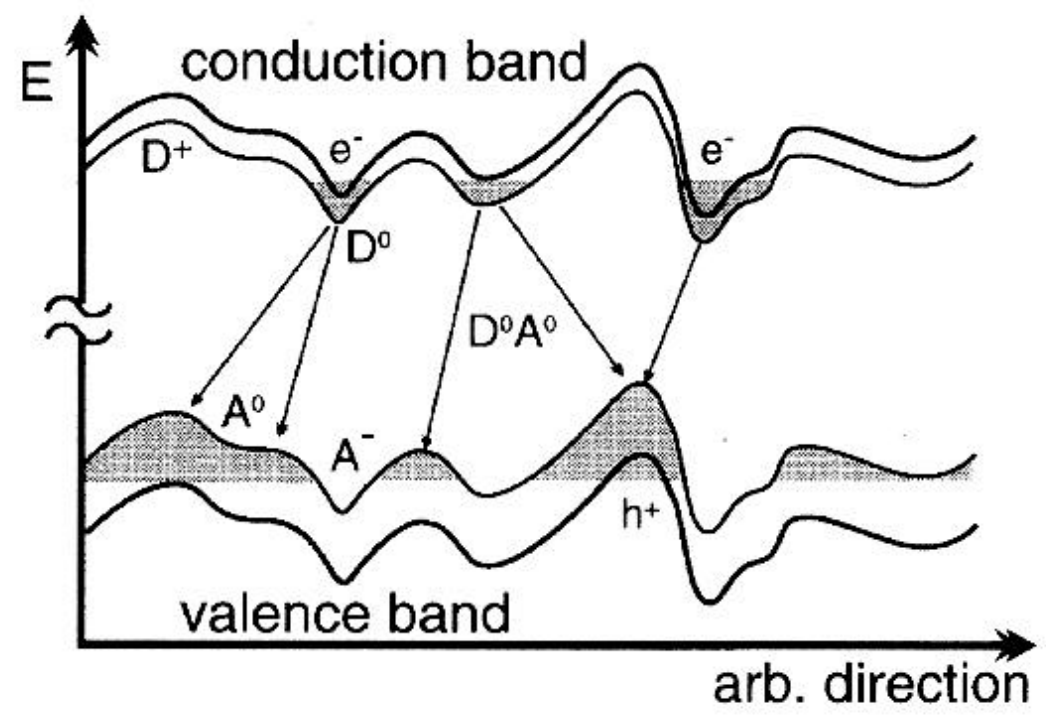

Figure 1-9 


\section{Chapter 2: Selective Excitation of the Yellow Luminescence of $\boldsymbol{n}$ - type GaN}

(Some of these results on the YL have previously been reported elsewhere [98].)

\subsection{Theory of Selective Excitation}

One difficulty in studying luminescent bands such as the YL is the very large width, around 500 $\mathrm{meV}$ for the $\mathrm{YL}$ in most samples. The technique of selective excitation is a well-established method for resolving narrower structures within a broad (inhomogeneously-broadened) spectrum. In selective excitation, also called resonant excitation, the excitation energy is chosen such that it is resonant with a narrow range of the broad emission energy. An inhomogeneously broadened spectrum is narrowed in this way by selecting out a homogeneous subset of the total spectrum. This technique has been applied successfully to a variety of materials, including ZnSe [99], CdSe [100], porous Si [101], Ge microcrystals [102], and GaInN [103].

In DAP emission, for example, inhomogeneous broadening arises from the Coulomb term in Equation (1.3), since the excitation and emission energies of the DAPs depend on the donor-acceptor separation distance. In normal, above band-gap excitation, emission includes contributions from DAPs of many different separations, and is thus broadened. Emission at a particular energy represents a subset of pairs whose separation fulfills Equation (1.3). Excitation at that same particular energy (as opposed to above band-gap excitation) correspondingly excites only that same subset of DAPs.

If there is no transfer of charges among donors and acceptors, then most of the emission from a selectively-excited sample will be at the same energy as (and therefore not separable from) the excitation. 
However, emission energy can also be shifted from the excitation energy for at least two reasons: (a) if a DAP is photoexcited into a higher energy electronic state and followed by relaxation into the ground state prior to recombination, or (b) if a phonon is emitted during the excitation or recombination process. In case (a), emission will be shifted by the excited state energy, and in case (b), emission will be shifted by the phonon energy. This is represented schematically in Figure 2.1. Note that selectively excited PL is rather similar to Raman scattering, in that the emitted (or scattered) photon energy is shifted by a constant amount shift from the excitation photon energy. These cases have been observed in DAPs by Tews, et al., (case A) [99] and by Yu and Hermann (case B) [100].

\subsection{Experimental Setup}

Our experiments of selectively exciting the YL were performed with a continuously tunable dye laser using the Coumarin 540 dye (with a tunable output between 2.1 and $2.35 \mathrm{eV}$ ). The dye laser was pumped by a high power, visible $\mathrm{Ar}^{+}$-ion laser. The fluorescence from the dye was prevented from reaching the sample and detector by filtering the dye emission (including laser line) with a diffraction grating. Some discrete lines between 2.4 and $2.7 \mathrm{eV}$ from a second visible $\mathrm{Ar}^{+}$-ion laser were used to extend the excitation range. A UV HeCd laser (at $325 \mathrm{~nm}, 3.814 \mathrm{eV}$ ) was used to measure the normal (with above band-gap excitation) photoluminescence. The temperature of the samples was varied between $12 \mathrm{~K}-300 \mathrm{~K}$ using a closed-cycle helium refrigerator. The PL signal was analyzed with a SPEX double spectrometer and detected with a cooled GaAs photomultiplier tube (PMT) connected to a photon-counting system. A schematic diagram of the experimental setup is provided in Figure 2-2.

\subsection{Samples}

Two types of samples were investigated in this experiment. One sample is a $2.65 \mu \mathrm{m}$ thick GaN film grown on sapphire by MOCVD. The film was heavily doped with $\mathrm{Si}$ donors $\left(\mathrm{Si}_{\mathrm{Ga}}\right)$. The room temperature carrier concentration from Hall-effect measurements was $5 \times 10^{18} \mathrm{~cm}^{-3}$. The sample was supplied by K. Uchida, from the Department of Communications and Systems, the University of ElectroCommunications, in Tokyo, Japan. 
The other two samples were bulk GaN samples, grown from the melt under high pressure. Details on their preparation have been reported elsewhere [104]. These were also heavily doped, although unintentionally, with oxygen donors $\left(\mathrm{O}_{\mathrm{N}}\right)$, and had room temperature carrier concentrations of around $5 \times$ $10^{19} \mathrm{~cm}^{-3}$. These samples were supplied by P. Perlin and I. Grzegory, from UNIPRESS, High Pressure Research Center of the Polish Academy of Sciences, in Warsaw, Poland.

Note that the shallow donor Bohr radius in GaN is approximately $23 \AA$, as discussed in section (1.1.2). The critical distance between shallow impurities $\left(r_{0}\right)$ in order to achieve a Mott metallic transition is theoretically given by $\lambda \sim 3$, where $\lambda=r_{0} / a$ ( $a=$ the impurity Bohr radius) [105], although experimentally lower values of $\lambda$ have been found (for example, $2<\lambda<2.5$ for $\mathrm{Zn}$ acceptors in $\mathrm{GaP}$ [106]). Therefore, one would expect the $\mathrm{GaN}$ metal-insulator transition to occur somewhere in the range of donor concentrations between $3 \times 10^{18} \mathrm{~cm}^{-3}$ to $1 \times 10^{19} \mathrm{~cm}^{-3}$ (corresponding to $\lambda=3$ and $\lambda=2$, respectively).

Keeping that in mind, the bulk samples we investigated are certainly metallic, whereas the film sample may or may not be beyond the metallic transition. An experimental study on Si-doped GaN found that for room temperature electron concentration of $n=7 \times 10^{18} \mathrm{~cm}^{-3}$, the sample investigated in that study was clearly above the metal-insulator transition, and for $n=1.6 \times 10^{18} \mathrm{~cm}^{-3}$, a second sample was close to the transition [107]. It seems likely, then, that our Si-doped film sample is also metallic.

\subsection{Experimental Results}

The YL of all samples is very similar, when excited above the band-gap with the HeCd laser. The $\mathrm{YL}$ is strong, with a peak around $2.2-2.3 \mathrm{eV}$, and a width of about $400-500 \mathrm{meV}$. This is also typical of other YL reports in the literature.

However, when excited resonantly, below the band-gap, the PL spectrum changes dramatically. Figure 2-3 compares the luminescence spectrum at $12 \mathrm{~K}$ obtained with above band-gap excitation, with a typical below band-gap resonantly excited spectrum (in this case, the $2.541 \mathrm{eV}$ line of an $\mathrm{Ar}^{+}$-ion laser was used). Several narrower features are observed, each peak having a width of about $100 \mathrm{meV}$.

This narrowing of the broad YL peak is observed in all samples. By deconvoluting the spectra into a sum of different peaks, the emission energies have been determined as a function of excitation energy. The deconvoluted spectrum is compared with the experimental spectrum in Figure 2-4, for one particular 
excitation energy $\left(E_{\mathrm{ex}}=2.471 \mathrm{eV}\right)$. A summary of all the peak positions obtained this way for many different excitation energies is shown in Figure 2-5. The film sample displayed a number of peaks (labeled "IF") not seen in the bulk samples, which were due to interference fringes produced by Fabry-Perot interference within the GaN film. These IF peaks complicated the curve-fitting process slightly, but were easily separated from the true emission peaks since the IF peaks did not vary with $\mathrm{E}_{\mathrm{ex}}$.

The emission peaks whose energy changed with $\mathrm{E}_{\mathrm{ex}}$ did so in a manner such that their separation from the excitation energy was a constant for each peak. These narrower peaks are labeled A, B', B, B", and $\mathrm{C}$ (peak $\mathrm{C}$ was not seen in the film sample). The energy shifts between emission and excitation for those peaks are 40,170, 200, 240, and $370 \mathrm{meV}$, respectively (with uncertainties of $\pm 10 \mathrm{meV}$ ).

The results from all three samples essentially agree within the experimental uncertainties. The exceptions are that the finer structures, B', and B", are not as clearly resolved in one bulk sample as in the other. Also, in the second bulk sample (not shown in Figure 2-5), peak A has a slightly larger energy separation than in the other two samples $(50 \pm 10 \mathrm{meV}$ as compared to $40 \pm 10 \mathrm{meV})$.

The temperature dependence of the selectively excited YL peaks was also measured, and the results for the second bulk sample are presented in Figure 2-6. The thermal quenching for temperatures above $\sim 150 \mathrm{~K}$ is in contrast to YL excited above the band-gap, where only a small dependence on temperature in this range has been seen $[68,90]$. A significant broadening of peaks A, B, and C, also occurred as the temperature increased, so that the narrowed structures are difficult to discern at room temperature.

Figure 2-7 displays the thermal quenching in the form of an Arrhenius plot, where the integrated intensity in a logarithmic scale is plotted as a function of the inverse temperature. The linear fit to the high temperature (left side) side of the Arrhenius plot typically yields an activation energy, determined by the slope. A more precise fit, which uses the entire temperature range, has been made to the data (and is displayed as the broken curves in Fig. 2-7), using the following formula:

$$
I=\frac{I_{0}}{1+B \cdot e^{-E_{A} / k_{B} T}}
$$


There are three adjustable parameters: $I_{o}, E_{A}$, and $B$. Equation (2.1) can be derived by assuming a simple 2level model containing a lower energy state and an excited state. The rate of change in the population of the excited state (labeled "2") can be expressed as:

$$
\frac{d N_{2}}{d t}=G-\frac{N_{2}}{\tau_{\text {rad }}}-\frac{N_{2}}{\tau_{\text {nonrad }}}
$$

where $N_{2}$ is the population of the upper state (assumed to be proportional to the emission intensity $I$ ), $G$ is the excitation rate, and $\tau_{\text {rad }}$ and $\tau_{\text {nonrad }}$ are respectively the radiative and non-radiative times for relaxing back into the lower (ground) state. Equation (2.1) is obtained as the steady-state solution to Equation (2.2), (i.e. $d N_{2} / d T=0$ ) if we assume that $G$ and $\tau_{\text {rad }}$ do not depend on temperature but that $\tau_{\text {nonrad }}$ has an activated temperature dependence given by:

$$
\frac{1}{\tau_{\text {nonrad }}}=A \cdot e^{-E_{A} / k_{B} T}
$$

Here, $E_{A}$ is the activation energy for an electron in level 2 to recombine non-radiatively and $A$ is the nonradiative recombination rate as $\mathrm{T}$ goes to infinity. Thus, $I_{0}=G \times \tau_{\text {rad }}$ and $B=A \times \tau_{\text {rad }}$ (i.e., $B$ is the ratio $\tau_{\text {rad }}$ to $\left.\tau_{\text {nonrad }}(\infty)\right)$.

The activation energies for the bulk sample obtained by fitting Fig. 2-7 in this way are 67, 57, and $73 \mathrm{meV}( \pm 10 \mathrm{meV})$ for peaks $\mathrm{A}, \mathrm{B}$, and C, respectively.

\subsection{Discussion}

The similarity between results from bulk and film samples is a strong indication that similar defects are involved in their selectively excited emission. It is also a good indication that the film sample shares a metallic nature with the bulk samples. Moreover, the observations that these narrower peaks are only seen when excited in resonance with the YL, and that their intensity tends to decrease strongly as the excitation energy decreases below $\sim 2.35 \mathrm{eV}$, suggest that these peaks are indeed related to the normal $\mathrm{YL}$ emission.

As mentioned above, in section 1.2, the most common view of the YL today is that it comes from a SD-DA recombination, produced by spatially distant donors and acceptors. That seems inconsistent with our results for two reasons. (1) In metallic samples such as ours, there should be a large amount of free 
electrons which would be available to recombine with the photo-excited deep acceptors; thus YL would not narrow as we have seen it. (2) The thermal quenching would still be dependent primarily on the acceptor binding energy and the samples would display a much greater activation energy than we have seen. The impurity band formed by the donors will ensure there are always free electrons and neutral donors which can recombine with all photo-excited holes on the acceptors, and if the YL were produced only by distant DAPs, the emission process would not necessarily involve the same donor as in the excitation process.

In order to explain our results, we propose an alternate model, in which the YL states that we are probing are localized DAP complexes. As discussed in section 1.2, many theoretical and experimental groups have proposed the involvement of donor-acceptor complexes in the DAP emission. Mostly the involvement of complexes has been in the context of comprising the deep donor/acceptor involved in a distant DAP process $[29,43,54,62]$. Nevertheless, some groups have proposed that the YL emission comes from localized DAP complexes, possibly involving extended defects, rather than distant DAPs $[55,64,66]$. We propose that such localized states are involved during selective excitation. However, additional contributions to the YL from distant DAPs may exist when excited above the band-gap; some possible reasons why these contributions may not affect YL when selectively-excited are discussed later in this work.

Within this model of localized DAP complexes, the inhomogeneous broadening which is narrowed by selective excitation does not arise from the Coulomb interaction. However, it is still necessary to assume that a large inhomogeneous broadening exists, resulting in a continuous or quasi-continuous density of states (DOS), since we are able to selectively excite the complexes over a large range of energies. This type of DOS could arise in a collection of complexes from fluctuations in energy levels due to variations in the distance or arrangement between the donor and acceptor, or variations in their surroundings. Neugebauer and Van de Walle, for instance, point out that there may be a large inhomogeneous broadening of defect complexes since defect levels can be broadened and shifted when the complexes are located near extended defects [54].

The narrower structures observed under selective excitation still have a large line width of about $100 \mathrm{meV}$, which presumably results from homogeneous broadening. The origin of this broadening at low temperatures is most likely from the strong electron-phonon interaction which is often present in strongly 
localized defects. A homogeneous broadening of $100 \mathrm{meV}$ contrasts with the assignment of at least one group, who suggested that the entire low temperature YL width $(\sim 500 \mathrm{meV})$ is due to electron-phonon interaction [63].

As discussed in section 2.1, the two typical ways of interpreting selectively-excited peaks shifted from the laser line are (a) photo-excitation into an excited state of the complex or (b) emission of a localized phonon. We attribute peak A to the latter process, since its energy shift of around $40 \mathrm{meV}$ is a plausible local vibrational frequency for $\mathrm{GaN}$. Moreover, the substitutional $\mathrm{Si}_{\mathrm{Ga}}$ and $\mathrm{O}_{\mathrm{N}}$ donors are both lighter than the host atoms they replace, and therefore can be expected to produce localized phonons known as "gap modes", which would appear in the gap between the acoustic and optical phonon branches [108]. Indeed, $40 \mathrm{meV}$ does lie in this gap [3], and is thus consistent with our assignment. On the other hand, the energy shifts of over $200 \mathrm{meV}$ for peaks B and C are too high for phonon modes. However, these energies are still plausible for excited electronic states associated with a deep acceptor, and therefore peaks B and C are attributed to excited electronic states of the DAP complexes.

Our assignment of YL emission arising from DAP complexes is at variance with some of the other observed YL phenomena discussed in section 1.2, which indicate the involvement of distant DAPs. To account for the discrepancy, we propose that there may be multiple recombination channels which can contribute to the YL, such as distant isolated donors and acceptors, distant isolated donors and acceptor complexes, localized donor-acceptor complexes, etc. The dominant channel may depend on many factors, such as (a) whether the YL is selectively excited (which tends to favor localized complexes), (b) whether excitation is above band-gap (distant DAP recombination becomes possible), (c) whether excitation is by high power-density light (may involve distant DAPs, free-to-bound, or transitions between complexes), etc. The possible recombination channels would also depend on the sample doping, temperature, and extended defects.

The existence of more than one recombination channel is a key to understand why the thermal properties of the YL depend on the excitation method. For example, when a highly $n$-type GaN sample is strongly excited in the normal way, with above band-gap photons, the large concentration of free electrons tends to favor recombination via distant DAPs or free-to-bound transitions. This channel is not expected to be strongly temperature dependent. On the other hand, during resonant excitation, DAP complexes will be 
excited directly as discussed. Electrons can then be thermally excited out of these complexes. Once delocalized, these electrons will have higher probabilities for being annihilated non-radiatively, which process can lead to thermal quenching. Thus, within our model, the measured activation energies of $\sim 60$ $70 \mathrm{meV}$ represent the depth of traps localizing the electron in the complex. (We attribute this localization energy to an electron rather than a hole, since the holes are expected to be bound much more strongly than this to the deep acceptors.)

As a final point, we note that if Ogino and Aoki's configuration coordinate model (discussed in section 1.2.1) were correct, we would expect to observe the same shift between emission and excitation energies described in Figure 1-6. Since no such shift was observed, this may be an argument against the validity of their CC model for the YL.

\subsection{Conclusion}

We have been able to resolve finer structures within the broad YL of strongly $n$-type GaN, both in thin film and in bulk samples. The similarity between the two types of samples indicates that the YL in each has a similar source. Unlike normal YL, excited above the band-gap, selectively excited YL displays a strong thermal quenching. Our results suggest that selectively excited YL involves recombination at localized DAP complexes, while YL excited in other ways may possibly involve distant DAPs. Characteristics of the DAP complexes within our model include (a) an electron localization energy of around $60-70 \mathrm{meV}$, (b) a localized phonon energy of around $40 \mathrm{meV}$, and (c) excited states of the complex at 200 and $370 \mathrm{meV}$ above the ground state. 


\section{Figure Captions for Chapter 2}

Fig. 2-1 Schematic diagram of the shift between excitation and emission energies in the case of selective excitation of spatially distant DAPs. $E_{e x}$ is the excitation energy, $E_{e m}$ is the emission energy, $R$ is the donor-acceptor separation distance, and the excited states of an acceptor are shown on the energy axis. Case (a) represents the shift between excitation and emission arising due to excitation into an excited state, case (b) represents the shift arising from emission of a localized phonon. Case (c) is normally excited DAP PL, with the emission broadened due to the dependence on $R$, according to Equation (1.3).

Fig. 2-2 Schematic diagram of the experimental setup for the selective excitation experiments.

Fig. 2-3 Comparison of the $12 \mathrm{~K}$ yellow luminescence of a bulk GaN sample between above band-gap excitation (solid curve) and selective excitation (broken curve).

Fig. 2-4 Examples of typical $12 \mathrm{~K}$ selectively-excited spectra in both (a) bulk and (b) film samples. The broken curves represent a deconvolution of the experimental (solid curve) spectrum into a sum of Lorentzians. The peaks labeled "IF" are caused by interference fringes and do not shift with the exciting laser energy; the other peaks (A, B, B', B", and C) all shift with the laser energy.

Fig. 2-5 Summary of the emission peak energies observed in the $12 \mathrm{~K}$ selectively excited YL of (a) a bulk GaN sample, and (b) the GaN film. The horizontal dashed lines in (b) are due to interference effects. Excitation energies between 2.1 and 2.35 were achieved with the dye laser, and excitation energies between 2.4 and 2.7 were achieved with the $\mathrm{Ar}^{+}$-ion laser. The energy differences between peaks $\mathrm{A}, \mathrm{B}$, and $\mathrm{C}$, and the laser line are 40, 200, and $370 \mathrm{meV}$, respectively.

Fig. 2-6 Temperature dependence of the selectively excited $\mathrm{YL}\left(\mathrm{E}_{\mathrm{ex}}=2.471 \mathrm{eV}\right)$ of a $\mathrm{GaN}$ bulk sample, for various temperatures between $20 \mathrm{~K}$ (top spectrum) and $250 \mathrm{~K}$ (bottom spectrum). 
Fig. 2-7 Arrhenius plot of the integrated intensity for the three peaks: A, B, and C. By fitting the experimental points to Equation (2.1), activation energies of 65,57 , and $73 \mathrm{meV}( \pm 10 \mathrm{meV})$ are obtained for peaks $\mathrm{A}, \mathrm{B}$, and $\mathrm{C}$, respectively. 

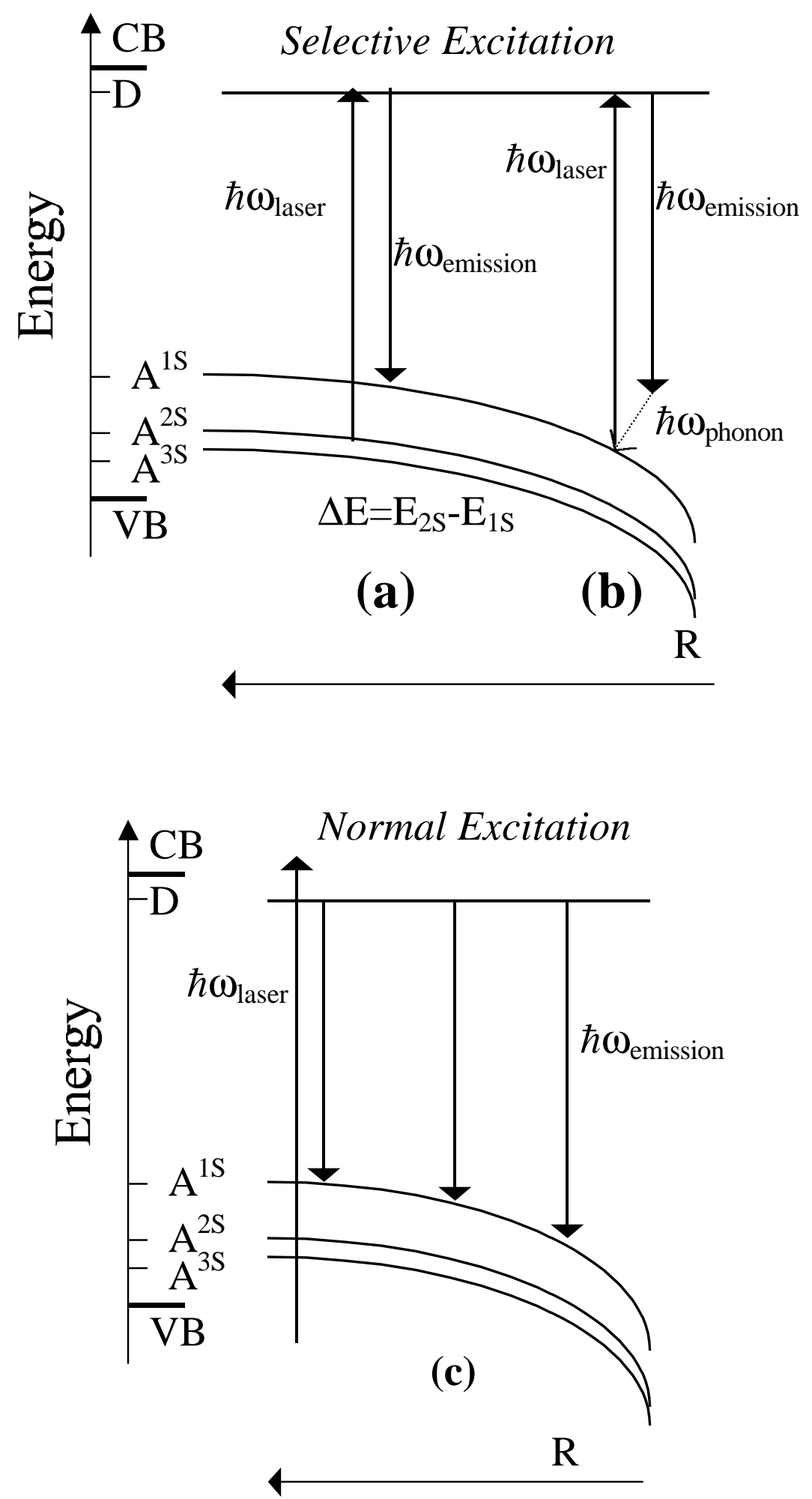

Figure 2-1 


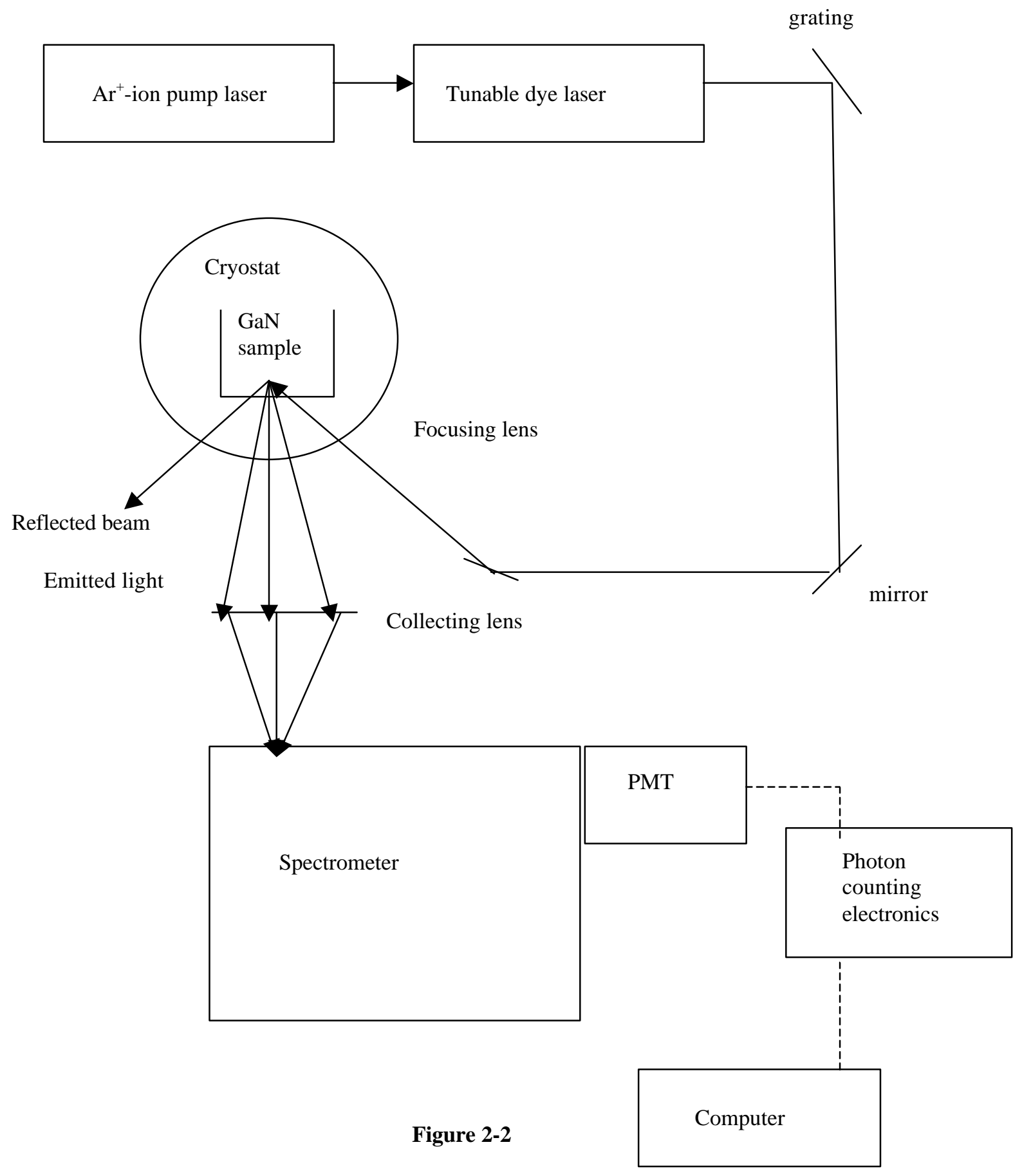




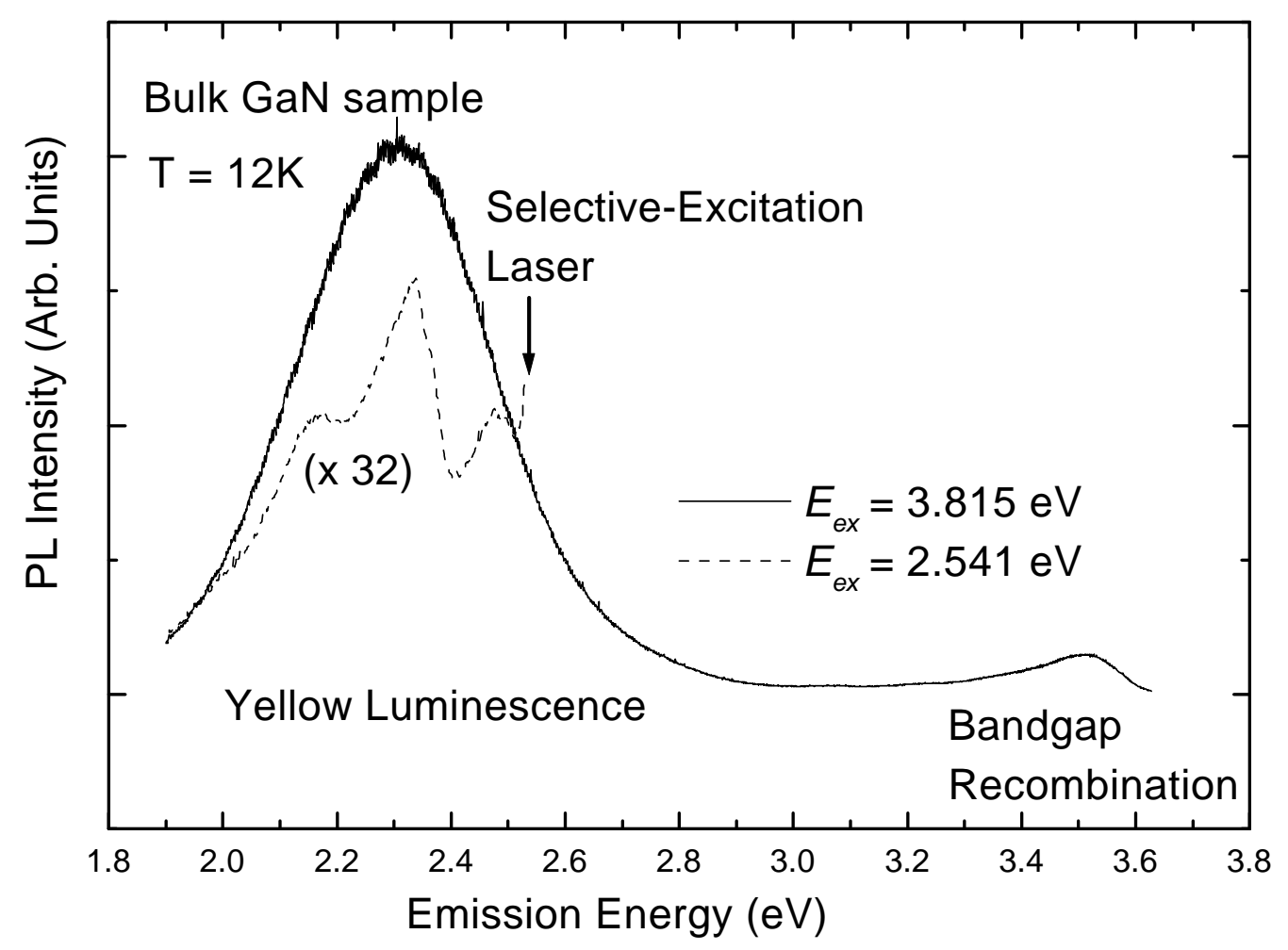

Figure 2-3 

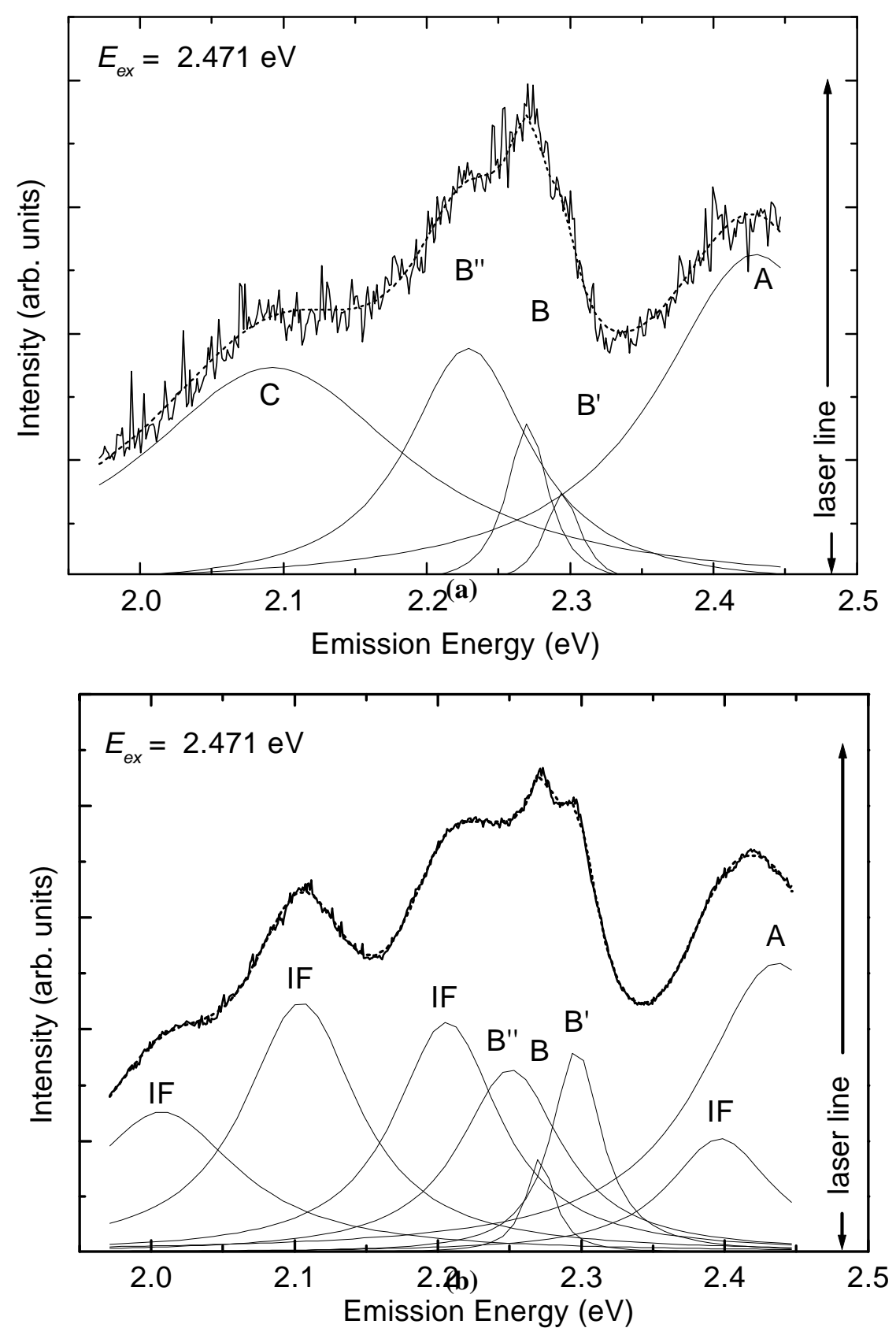

Figure 2-4 


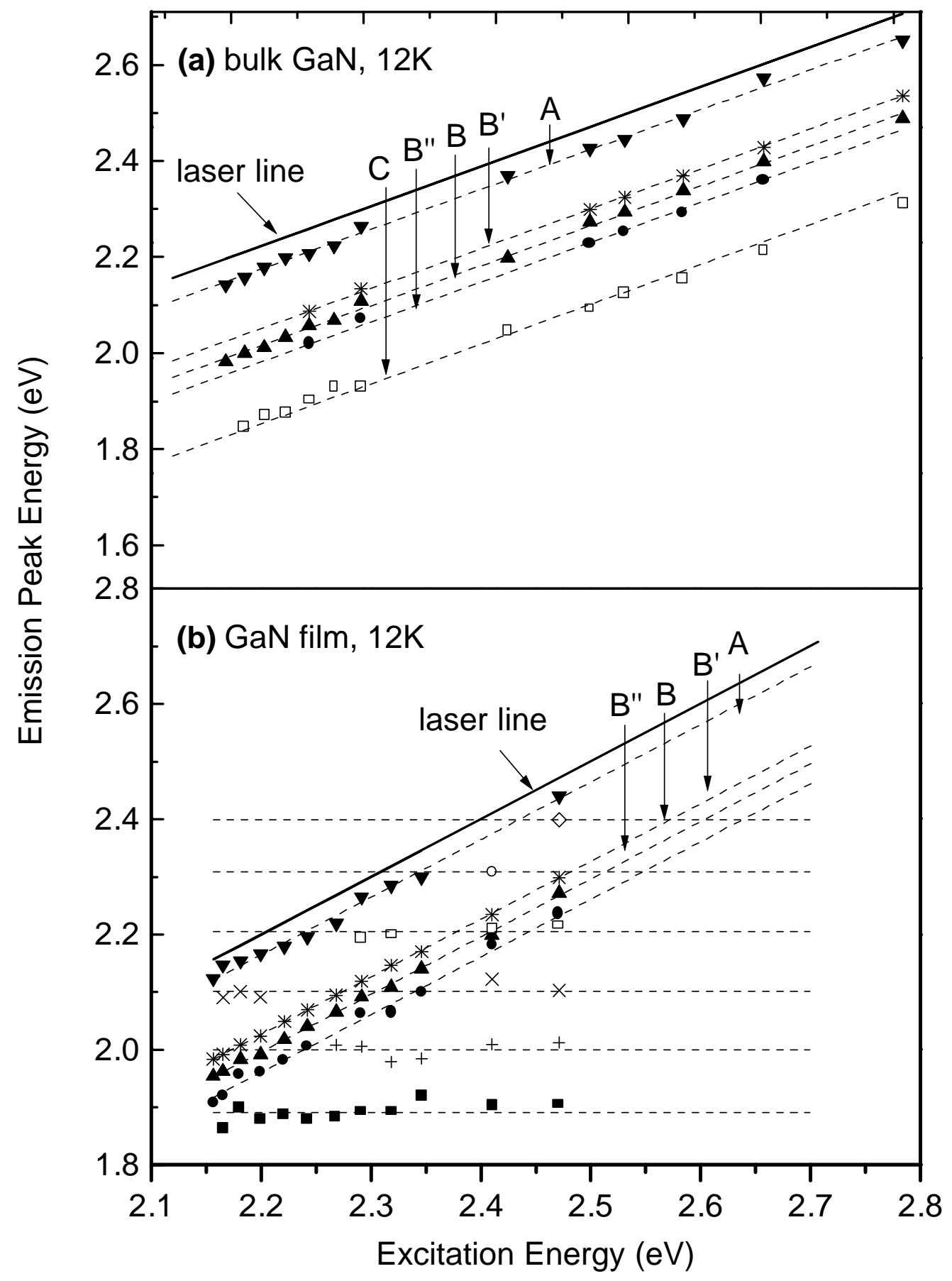

Figure 2-5 


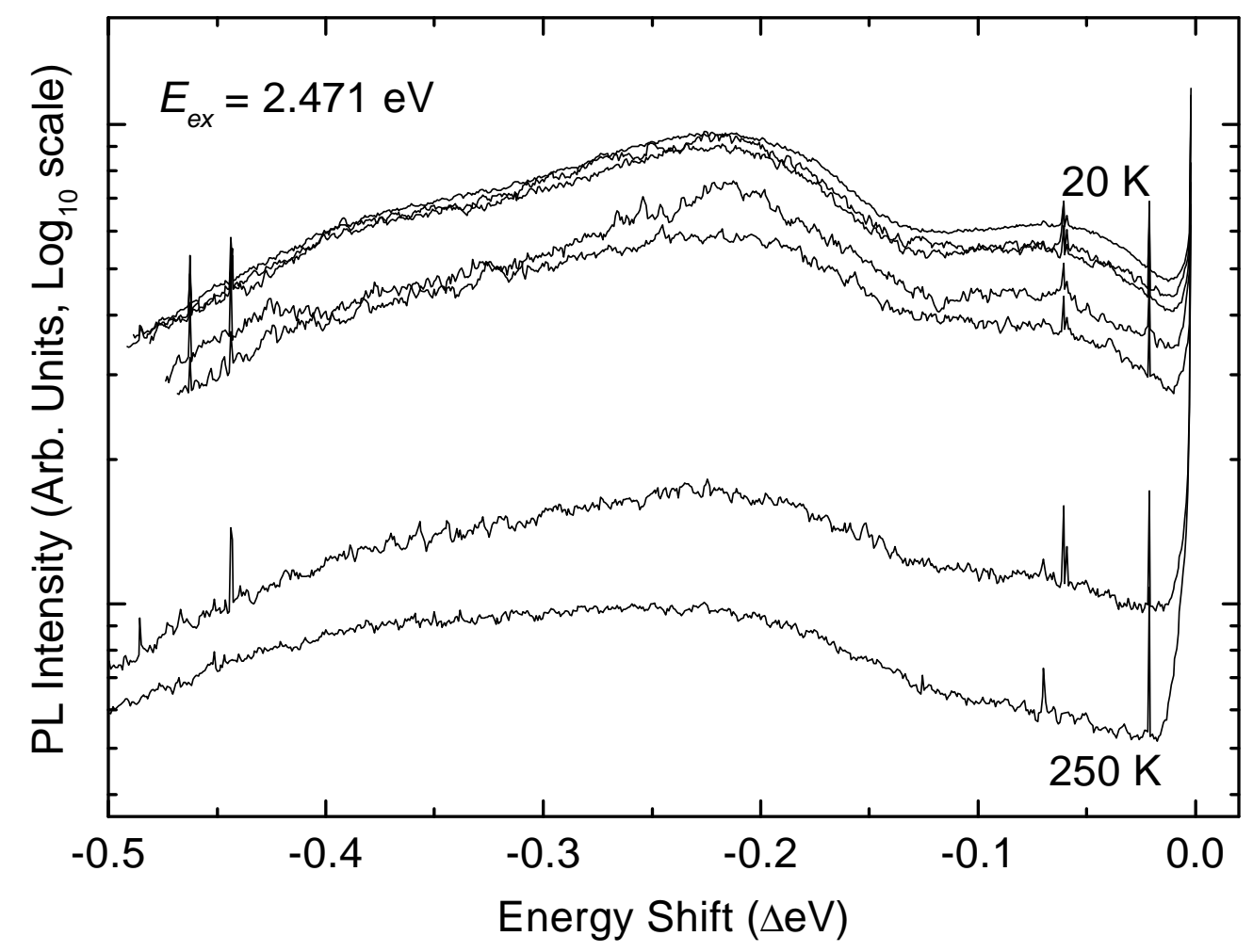

Figure 2-6 


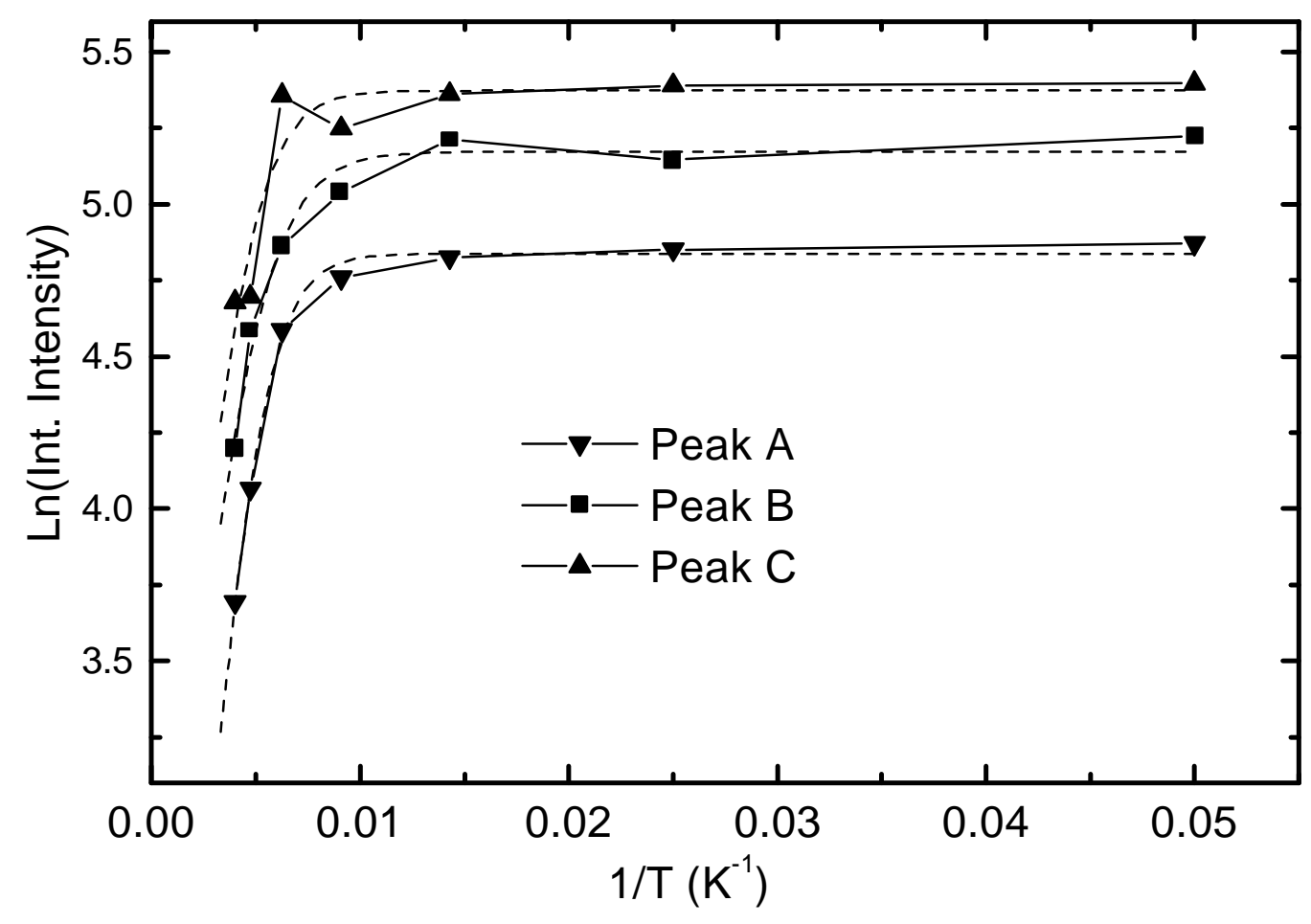

Figure 2-7 


\section{Chapter 3: Selective Excitation of the Blue Luminescence of Mg- doped $p$-type GaN}

As with the YL, the large width of the BL has made it difficult to study the nature of the emission. We have therefore employed selective excitation, as in the case of the YL, to learn more about the BL emission.

\subsection{Experimental Setup}

The experimental setup is much like that of the YL experiment (described in section 2.2; see Figure 2-2). A different dye was used to resonantly excite the BL: Stilbene 420 (having a tunable output between $2.67 \mathrm{eV}$ and $3 \mathrm{eV}$ ). This necessitated replacing the pump laser with a $\mathrm{UV}^{+} \mathrm{Ar}^{+}$-ion laser, which had $2.5-3.0 \mathrm{~W}$ of power spread across several UV lines. A diffraction grating was used, as in the YL experiment, to filter out the fluorescence from the dye. Again, the UV HeCd laser (at $325 \mathrm{~nm}, 3.814 \mathrm{eV}$ ) was used to measure the normal photoluminescence (with above band-gap excitation). The temperature control, spectrometer, and detector were also the same as in the YL experiment.

\subsection{Sample}

The results discussed below were based on experiments performed on one sample, the one from among several measured Mg-doped GaN samples which showed the highest level of BL signal. This sample was grown via MOCVD on a sapphire substrate. It was annealed at $750{ }^{\circ} \mathrm{C}$ for 4 minutes after growth. The sample is $p$-type, with a room temperature carrier concentration of $2 \times 10^{17} \mathrm{~cm}^{-3}$. In many respects (annealing temperature, carrier concentration, luminescence), this sample is very similar to those used by Götz, et al. [77]. Using Götz, et al.'s values for samples annealed at 700 and $775{ }^{\circ} \mathrm{C}$ as guidelines, it seems likely that our sample has the following characteristics: acceptor concentration $[\mathrm{A}] \sim 2 \times 10^{19} \mathrm{~cm}^{-3}$, and compensating donor concentration $[D] \sim 4 \times 10^{18} \mathrm{~cm}^{-3}$. This is a compensation ratio of $K=0.2(20 \%)$, which is similar to the value reported for Kaufmann, et al.'s samples $(K=0.3)$ [63]. 
As mentioned in section 2.3, the Mott transition for shallow donors occurs in GaN for a shallow donor concentration of around $3-5 \times 10^{18} \mathrm{~cm}^{-3}$. It seems likely then, that if shallow donors are responsible for the compensation, the sample will be near the metallic transition (provided the donors are in their neutral state).

Conversely, the Mott transition for shallow acceptors occurs at a much higher concentration of acceptors, due to the much smaller Bohr radius for acceptors. Using the value of $4 \AA$ for the shallow acceptor Bohr radius, as discussed in section 1.1.3, and the arguments in section 2.3, one finds that the acceptor Mott transition should occur for acceptor concentrations around $1-5 \times 10^{21} \mathrm{~cm}^{-3}$. Therefore, the acceptor levels are clearly below the Mott transition.

While the compensation ratio of $K=0.2$ is significant, it does not put the sample into the regime of "highly compensated" as defined by Shkloskii and Efros, strictly speaking. However, to serve as approximate guides, the values of $r_{s}$ (screening radius), $\gamma$ (average fluctuation amplitude), and $R_{q}$ (short range well width) are calculated based on the room-temperature carrier concentration and the above estimated donor/acceptor concentration values: $r_{s}=80 \mathrm{~nm}, \gamma=200 \mathrm{meV}$, and $R_{q}=2 \mathrm{~nm}$, Of course, photoexcitation and temperature variation can increase the carrier concentration (and hence screening) and correspondingly change these values.

\subsection{Experimental Results}

The "normal" BL of our sample, when excited above the band-gap by the HeCd laser, appears to be very similar to the BL of other samples reported in the literature, and is plotted in Figure $3-1$. The BL is centered around $2.8 \mathrm{eV}$, and is about $300 \mathrm{meV}$ wide. The small oscillations that modulate the envelope of the BL are (as in the YL film sample) the result of Fabry-Perot interference between the two surfaces of the GaN film.

Unlike the YL, the BL does not show a significant narrowing when excited selectively, and no fine structures are seen. A representative selectively excited spectrum is shown together with the normal BL in Figure 3-2. The peak remains relatively broad (having a width of $\sim 200 \mathrm{meV}$ ), and is shifted in energy from the laser line as well as from the normal BL position. Oscillations from Fabry-Perot interference remain visible. 
Figure 3-3 presents some representative selectively excited BL spectra, plotted in a semi-log plot as a function of the difference between the excitation and emission energies. In contrast to the YL (Figure 2-5), a simple dependence of peak position on excitation energy was not found. Instead, we observed a rather complicated dependence, characterized by two regimes: (1) For excitation energies less than $2.8 \mathrm{eV}$, a constant energy separation of about $180 \mathrm{meV}( \pm 10 \mathrm{meV})$ between excitation and emission was found. This regime is reminiscent of the selectively-excited YL results. (2) For excitation energies greater than 2.8 $\mathrm{eV}$, the BL peak position varied linearly with the excitation energy, with a slope of about $0.57( \pm .05)$. These results are plotted in Figure 3-4. Note that the y-axis in the figure is the energy difference, not the absolute position, so the slope on the graph for excitation energies greater than $2.8 \mathrm{eV}$ is equal to -0.43 rather than 0.57 .

In addition to the obvious shift in peak positions, another noticeable feature of Fig. 3-3 is the change in the BL intensity. As the excitation energy was decreased, so was the emission intensity. The integrated intensity of the selectively excited BL is plotted as a function of excitation energy in Figure 3-5. As shown by the straight line on this semi-log plot, the intensity ( $I$ ) grows exponentially with increasing excitation photon energy $\left(E_{e x}\right)$. A least squares fit reveals that $I \sim \exp \left(E_{e x} / E_{0}\right)$, with $E_{0}=33 \mathrm{meV}( \pm 5 \mathrm{meV})$.

The temperature dependence of the selectively-excited BL has also been investigated, and is presented in Figure 3-6 for one particular excitation energy, $E_{e x}=2.887 \mathrm{eV}$. The luminescence shows an activated thermal quenching. This can be depicted in an Arrhenius plot, similar to the one described in section 2.4. The plot, together with a fit to Equation (2.1) is given in Figure 3-7. The value of the activation energy obtained from the fit, is $E^{*}=22 \mathrm{meV}( \pm 3 \mathrm{meV})$. We have performed similar experiments with different excitation energies, and have found that this activation energy is fairly constant, with $E^{*}=22 \pm 5$ $\mathrm{meV}$ for $2.8 \mathrm{eV} \leq E_{e x} \leq 3.0 \mathrm{eV}$.

This temperature dependence contrasts sharply with published values of the activation energy for thermal quenching of the BL, when excited by normal, above the band-gap photons. For example, Reshchikov, et al., found that the BL had an activation energy of $300-400 \mathrm{meV}$, with thermal quenching becoming significant only for temperatures above $250 \mathrm{~K}$ [76]. The large difference between activation energies obtained through resonant vs. above band-gap excitation is reminiscent of the YL experiments discussed Chapter 2. However, it should perhaps be noted that at least one group has reported thermal 
quenching at much lower temperatures (a quenching of a factor of 50 in going from low T to 300 K) [96]. It is not clear, though, why the result of that group should differ so much with the results of two other groups, which reported a quenching beginning at much higher temperatures, and a total quenching of only a factor of 10 in going from low $\mathrm{T}$ to $300 \mathrm{~K}[63,76]$.

\subsection{Discussion}

There are essentially five pieces of experimental evidence which must be explained by a model for the selectively-excited BL. These are (1) the exponential dependence of BL intensity on excitation energy, (2) the behavior of the BL peak position for $E_{e x}<2.8 \mathrm{eV}$, (3) the change in peak position behavior around $E_{e x}=2.8 \mathrm{eV},(4)$ the behavior of the BL peak position for $E_{e x}>2.8$, and (5) the thermal quenching of the BL. Those experimental results will be discussed in that order, in the following subsections. We find that our results can best be explained by a model which involves large potential fluctuations (as discussed in section 1.3.2) and a large amount of disorder produced by the high doping.

\subsubsection{Band-Tailing}

As noted in the section 3.3, the intensity (I) grows exponentially with the excitation energy $\left(E_{e x}\right)$, $I \sim \exp \left(E_{e x} / E_{0}\right)$, with $E_{0} \approx 33 \mathrm{meV}$. This kind of exponential behavior is reminiscent of the "Urbach tail", an exponentially increasing absorption edge near and below the band gap seen in many disordered materials [109]. The energy, $E_{0}$, is known as the Urbach parameter. The Urbach tail is usually associated with an exponential density of states, induced by disorder which can be produced by doping or crystal defects. Sometimes the term "Lifshitz tail" is used to describe the more general case of a tail of states extending into the band gap due to defects or disorder (as opposed to the exponential absorption edge associated with the "Urbach tail") [110]. Amorphous semiconductors, for example, are well-known to contain band tail states. Highly doped semiconductors, due to the disorder created by doping, often display properties similar to the amorphous materials. Since Mg-doped GaN is not only highly doped, but also self-compensated to some degree, the presence of a large band tail is not entirely unexpected. Correspondingly, we interpret the measured exponential dependence in the BL intensity to reflect the absorption coefficient, and hence is a measure of the band tail. If the absorption coefficient increases exponentially with photon energy, then as 
higher energy photons are used to selectively excite the BL, an exponentially greater number of them will be absorbed. Consequently, an exponentially larger luminescence will be measured. Based on this interpretation, our measured $E_{0}$ corresponds to the Urbach parameter, and is the width of tail states.

Observation of band tails in $\mathrm{Mg}$-doped $\mathrm{GaN}$ has previously been reported by only two groups, as far as we know. Kang, et al., for example, found a thermally induced blue-shift of the bound exciton peaks and concluded that the positions and intensities of exciton peaks are significantly affected by band-tailing produced by potential fluctuations in their Mg-doped samples [111]. Although their samples did not display the BL, they believed their samples were highly compensated. No numerical value of the band tail parameter was deduced in this experiment.

Secondly, Qiu, et al., observed band-tails in $n$-type and $p$-type GaN through photoconductivity (PC) measurements [112]. Their results are quite interesting. They concluded from their PC measurements that the absorption coefficients in both $n$-type and $p$-type samples increase with excitation energy $\left(E_{e x}\right)$ as $\exp \left(E_{e x} / E_{0}\right)$, when $E_{e x}$ is between 1.5 and $3.0 \mathrm{eV}$. This is very similar to our results. However, their value for $E_{0}$ was quite large: $180-220 \mathrm{meV}$ for the $\mathrm{Mg}$-doped $p$-type samples (with a nearly as large value for their nominally undoped $n$-type samples). They suggested that as Mg-doping is increased, the band-tailing shifts from being predominantly in the valence band (in $n$-type $\mathrm{GaN}$ ) to predominantly in the conduction band (for $p$-type $\mathrm{GaN}$ ). A narrower tail was measured in some samples (with $E_{0} \sim 50 \mathrm{meV}$ ) and the authors suggested that there may be two exponential band tails in all of their samples: a narrow one which is present in both $\mathrm{CB}$ and $\mathrm{VB}$, and a broad one which for $p$-type GaN is only seen in the $\mathrm{CB}$. The authors proposed that the sharper tail may be associated with the usual Urbach edge, as a result of perturbations of the band structure by potential fluctuations. The broader tail was tentatively attributed to "defect states". We note that Chichibu, et al., have reported a much smaller value for the Urbach parameter in nominally undoped GaN samples, with $E_{0} \sim 6-12 \mathrm{meV}$ [113].

The large difference between our value for the band tail parameter in $\mathrm{Mg}$-doped $\mathrm{GaN}\left(E_{0} \sim 33\right.$ $\mathrm{meV})$ and the value of Qiu, et al., $\left(\mathrm{E}_{0} \sim 200 \mathrm{meV}\right)$ may arise from various factors. First, the samples themselves were different. Since they reported neither annealing temperature nor photoluminescence spectra of their two $p$-type samples, but only hole concentrations of $6 \times 10^{17} \mathrm{~cm}^{-3}$ and $7 \times 10^{16} \mathrm{~cm}^{-3}$, it is difficult to say whether their films are similar in terms of acceptors and degree of compensation to our film. 
Secondly, if the band tails as measured by Qiu, et al., are applicable to our sample, it would appear that we are seeing the sharper tail only (their value of $E_{0} \sim 50 \mathrm{meV}$ is not too different from our value of $33 \mathrm{meV}$ ). This would suggest that the BL originates from shallow donors and shallow acceptors which are modified by the large potential fluctuations rather than from deep defect levels.

\subsubsection{Frank-Condon Shift and Electron-Phonon Coupling}

As described in section 2.1, the normal behavior of an inhomogeneously broadened emission, when selectively excited, is for the peak position to change with the excitation energy, as in Raman scattering. This was observed for the YL, as reported in Chapter 2. The energy separation between the excitation and emission energies is then interpreted to correspond to either a phonon energy or an excited state energy of donors or acceptors. Conversely, in the case of non-resonant excitation, we do not expect the peak position to change with the excitation energy. Thus, the two typical dependences of the peak position on the excitation energy are either a constant peak position or a constant shift. However, that is not what we observe with the selectively excited BL. As noted in section 3.3, our results show two regimes: a constant shift of $180 \mathrm{meV}$ for $E_{e x}<2.8 \mathrm{eV}$, and a linear dependence for $E_{e x}>2.8 \mathrm{eV}$. This section will discuss the behavior of the BL in the first regime, $E_{e x}<2.8 \mathrm{eV}$.

First, though, we point out that as discussed in section 2.5, when the YL was selectively excited, a much narrower line width was observed as compared to the above band-gap excited YL. This result led to the conclusion (different than Kaufmann, et al. [63]) that most of the low temperature broadening observed in the YL is inhomogeneous rather than homogeneous. The BL, in contrast, did not exhibit a large narrowing when selectively excited (in both regimes). This supports Kaufmann, et al.'s interpretation of the low temperature BL width as being homogeneous, due to electron-phonon coupling [63].

Along with that interpretation, Kaufmann, et al., also estimated the Frank-Condon shift of the BL to be $180 \mathrm{meV}$. A Frank-Condon shift (sometimes also called the Stokes shift) is typically the term given to describe the difference between absorption and emission in a configuration coordinate (CC) model [114], such as was described for the YL in section 1.2.1 (see Figure 1-6). It arises due to a difference in the local configuration of the atoms (possibly host as well as impurities) in the ground and excited states of a defect center. Since the electronic transitions occur in a time scale much shorter than the physical rearrangement 
of the atomic configuration, optical transitions between ground and excited states take place in a different configuration for absorption as compared to emission. As shown in Figure 1-6, this can lead to an energy shift between absorption and emission.

If such a Frank-Condon shift is indeed present in a defect center, it should also be seen in the selective excitation spectra, provided the time for recombination is longer than the lattice relaxation time (and provided the carriers do not hop to different defect centers). Thus, the $180 \mathrm{meV}$ peak shift we have seen for $\mathrm{E}_{\mathrm{ex}}<2.8 \mathrm{eV}$ is consistent with the Frank-Condon shift as proposed by Kaufmann, et al. We note that this value is approximately equal to two bulk LO phonons in GaN, and it suggests that the resonantly excited defects interact strongly with the bulk LO phonons.

\subsubsection{Mobility Edge}

Highly doped semiconductors have similarities with amorphous semiconductors, in that both materials contain a high degree of disorder. The existence of band tails (discussed in section 3.4.1), is one example of that similarity. The structural disorder in amorphous semiconductors is known to produce additional important effects on the transport properties, in the form of localization and the existence of a mobility edge. We will show that our selective excitation results can be explained by the presence of a similar mobility edge in our highly Mg-doped $p$-type GaN sample, presumably produced by the disorder inherent in the large amount of Mg doping.

The BL response in the $E_{e x}>2.8 \mathrm{eV}$ regime is actually quite unusual, in that the peak position does not follow either of the two typical behaviors. As discussed in sections

2.1 and 3.4.2, the two typical behaviors for emission are either a constant peak position, or a constant shift with excitation energy. The unusual behavior of the BL peak position in this regime is highlighted in Figure 3-4, where for $E_{e x}>2.8 \mathrm{eV}$ the two typical behaviors are plotted as broken lines (labeled "PL" and "Raman" accordingly).

To interpret the behavior for $E_{e x}>2.8 \mathrm{eV}$, it is useful to understand a difference between Raman scattering and photoluminescence. When photons are used to excite Raman scattering, the photons couple mainly to electrons in the semiconductor, and the photon-phonon scattering is thus mediated by an 
electron-hole pair, which usually is virtual [115]. The scattering process occurs rapidly, and the electronhole pair is unchanged by the process. In photoluminescence (PL), however, there must be excitation of a real electron-hole pair. Relaxation of the electron and/or hole into a lower energy state may occur prior to recombination, in which case the energy lost in relaxation is equal to the difference between excitation and emission. Our results imply that for $E_{e x}<2.8 \mathrm{eV}$, no relaxation takes place before the LO-assisted radiative recombination, but for $E_{e x}>2.8 \mathrm{eV}$, the electron-hole pair states begin to lose energy in relaxation to lower band-tail states before recombination takes place.

Based on a DAP model, an electron and hole are photoexcited at particular donors and acceptors. After excitation, the electrons or holes may or may not "hop" to a different donor/acceptor with lower energy before recombination. Such hopping among defect states can give rise to a contribution to the total electrical conductivity known as "hopping conductivity" (i.e., tunneling from impurity center to impurity center). Hopping conductivity is well known to be important in highly doped materials [116]. Our results suggest that for $E_{e x}<2.8 \mathrm{eV}$, no hopping occurs (or that the hopping time compared to recombination time is long), but that for $E_{e x}>2.8 \mathrm{eV}$, hopping becomes faster. In other words, there is a variation in the hopping time dependent on the energy of the involved donor and acceptor states, with a transition between short and long hopping times occurring at $E_{e x}=2.8 \mathrm{eV}$. This model is depicted schematically in Figure 3-8. Note that in this model, recombination for $E_{e x}>2.8 \mathrm{eV}$ is PL-like, since the photoexcited carriers relax into lower energy states before recombination. The situation for $E_{e x}<2.8 \mathrm{eV}$, when the hopping or relaxation time is longer than the recombination time, means the emission is more Raman-like. We propose that the variation in hopping times is due to stronger localization of carriers for $E_{e x}<2.8 \mathrm{eV}$, and will discuss that below.

A transport time which depends on the energy of the carriers is precisely what occurs in amorphous semiconductors, and is known to give rise to a "mobility edge". Amorphous semiconductors form localized states deep in the band-tail, but extended states in the higher energy part of the tail. There is a sudden change in transport properties between the higher and lower energy states. Since the density of states changes continuously with energy in the band-tail, the sudden change in transport properties arises from a sudden change in the mobilities, with the localized states having low mobilities and the extended states having higher mobilities. The energy where this sudden change occurs is called the "mobility edge". 
The mobility edge determines which carriers may contribute to the conduction. In hydrogenated amorphous silicon, for example, time-of-flight experiments from optically injected carriers have shown that charge transport can be described by a model in which the electrical conduction takes place only via carriers above the mobility edge. Carriers below the mobility edge cannot conduct unless they are thermally excited into states above the mobility edge [117]. (It is interesting to note that the Urbach parameter for that material given in reference 117 is $E_{0} \sim 30-40 \mathrm{meV}$, which is the same order of magnitude as the value we obtained for Mg-doped GaN.) In the amorphous semiconductors, it is common to discuss the "mobility gap" (i.e., the energy separation between the mobility edges for electrons and holes) instead of the "bandgap", since it is the important energy gap for charge transport [118].

In analogy with amorphous semiconductors, we propose that we are observing a similar mobility gap in Mg-doped p-type GaN, with this gap occurring around $2.8 \mathrm{eV}$. Photoexcited electron-hole pairs with energies less than $2.8 \mathrm{eV}$ are localized, and do not relax. Emission from such carriers takes place from the same DAP which is optically excited and behaves like Raman scattering with $180 \mathrm{meV}$ phonons. Carriers excited into two-particle states with energies greater than $2.8 \mathrm{eV}$ are delocalized, and may relax some of their energy via hopping into lower energy DAPs. Observed emission in that case takes place from DAPs that are different (lower in energy) from the ones originally optically excited, and is PL-like. As a result, we observe the increased peak shift between excitation and emission, which depends on excitation energy.

\subsubsection{Slope of $E_{e m}$ vs. $E_{e x}$ for $E_{e x}>2.8 \mathrm{eV}$}

The energy relaxation between DAPs is difficult to quantify, and hence we have not been able to find a quantitative theory to explain the observed slope of $\sim 0.57$, relating the emission energy to the excitation energy in the $E_{e x}>2.8 \mathrm{eV}$ regime. As a qualitative explanation of why this slope deviates from the two expected cases of either a slope of unity or a slope of zero, we reiterate the notion stated in section 3.4.3, that the emission in this regime is PL-like, in that the carriers relax before recombination. In PL, carriers normally relax to a state at a particular energy, typically with a lifetime dominated by radiative recombination. As a result, the PL emission is normally constant and independent of the excitation energy. We believe the blue-shift in emission with respect to excitation energy observed in our experiments is 
related to the increasing number of carriers present at higher excitation energies, due to the exponential absorption edge.

In a DAP transition, as mentioned in section 1.2.1, a blue shift in emission with increasing excitation intensity may be observed, with the standard explanation being that as more carriers are excited, the donors and acceptors involved in recombination tend to be closer to each other, and the Coulomb term in equation (1.3) causes a blue-shift in emission. Although this explains our observed blue-shift, this effect will not predict a simple linear relationship between the emission energy and the excitation photon energy.

We propose that the blue-shift can be explained by combined effects of two mechanisms. This first one is due to the screening of the potential fluctuations. As described in section 1.3.2, two of the quantities which describe the potential fluctuations are a screening radius and an average amplitude of potential variation. When screening of the fluctuations increases due to increased free carrier concentration (for example, when excitation intensity is increased), the screening radius decreases and the amplitude of potential variation decreases. As a result, a blue-shift in the PL emission occurs. This is the reverse of what happens in Figure 1.9 (b), where a red-shift in the PL emission due to increased fluctuations is depicted. In our experiment, the exponential absorption edge means that increasing the photon energy of the excitation laser has the effect of increasing the free carrier concentration. Therefore, higher excitation energies result in more screening of the potential fluctuations. The blue-shift with increasing excitation energy that we observe in the $E_{e x}>2.8 \mathrm{eV}$ regime, therefore, has the same origin as the blue-shift with increased excitation intensity seen in materials with strong potential fluctuations.

The second mechanism that can give rise to a blue-shift is band filling. Band filling is a wellknown effect whereby the Fermi level of electrons or holes is moved towards the band (CB or VB, respectively) due to the filling up of the lower available states by carriers excited by optical excitation. Normally band filling is a significant effect only when the free carrier concentration is sufficiently high that the quasi-Fermi levels of the electrons or holes lie within the band, but it is impossible to rule out this effect as a contributor to the blue-shift with excitation energy in our sample.

These effects have been investigated by Kuskovsky, et al., by using a fixed excitation energy while changing the excitation intensity $(I)$ in heavily $\mathrm{N}$-doped $\mathrm{ZnSe}$ [119]. They found a nearly linear 
dependence of the DAP emission peak position on the log of excitation intensity in heavily doped materials:

$$
E_{e m}=A \log _{10}(I)+\text { constant }
$$

where $E_{e m}$ is the energy of the emission peak and $A$ is a constant. Kuskovsky, et al., have explained their results via a quantitative model in which the quasi-Fermi level of the minority carriers (in what they have labeled as the "low excitation intensity approximation") changes in a self-consistent way as a function of screening effects and excitation intensity. Similar linear dependence of the emission peak energy on the logarithm of the excitation intensity has likewise been reported for the BL by at least two two groups: Reshchikov, et al. [76], and Kaufmann, et al. [75]. Their results indicate that the constant $A$ has a value of $57 \mathrm{meV}$ per decade and $82 \mathrm{meV}$ per decade, respectively.

Equation (3.1) is consistent with our own observation of a linear dependence of peak position on excitation energy for $E_{e x}>2.8 \mathrm{eV}$. If we assume that the number of excited carriers $(N)$ in the Kuskovsky, et al., experiment is proportional to the excitation intensity:

$$
N \propto I
$$

then their result can be expressed as:

$$
E_{e m}=A \log _{10}(N)+\text { constant }
$$

From our observed exponential dependence of the BL intensity on the excitation photon energy, $E_{e x}$, we propose that the photoexcited carrier concentration, $N$, in our sample, dependes on $E_{e x}$ as:

$$
N=B \exp \left(E_{e x} / E_{0}\right)
$$

where $E_{0}$ is the Urbach parameter and $B$ is a proportionality constant. Thus, our experiment is similar to that of Kuskovsky, et al., in that $N$ is varied in both experiments. The experiments are different in that we change $N$ by varying $E_{e x}$ while Kuskovsky, et al., vary $I$.

Combining equation (3.4) with equation (3.3), we find:

$$
\begin{aligned}
& E_{e m}=A \log _{10}\left(B \exp \left(E_{e x} / E_{0}\right)\right)+\text { constant } \\
& E_{e m}=A \log _{10}(\mathrm{e}) / E_{0} \times E_{e x}+\text { constant }
\end{aligned}
$$

This describes a linear dependence of emission energy on excitation energy, such as we observed, with the slope equal to $A \log _{10}(\mathrm{e}) / E_{0}$. The constant $A$ is difficult to compute theoretically for our experimental conditions since it depends on several unknown material parameters. However, based on our measurement 
of the slope of 0.57 , equation (3.5) predicts a value of $A=43 \mathrm{meV}$ per decade. This value is consistent with the values of $A$ for the BL given by Kaufmann, et al. [75], and is in good agreement with the value obtained by Reshchikov, et al. [76]. Thus, we consider this as evidence that the observed peak position change for $E_{e x}>2.8 \mathrm{eV}$ can be explained by the increased number of carriers within our potential fluctuation model.

\subsubsection{Temperature Dependence}

Within our model, it is not entirely clear what the activation energy obtained from temperature dependent experiments represents, although the small value we obtained $(\sim 20-30 \mathrm{meV})$ is suggestive of the involvement of shallow donors rather than deep donors in the selectively excited BL. In the mobility gap model, described in section 3.4.2, the main effect of raising the temperature is to make hopping possible for localized states lying within $\mathrm{k}_{\mathrm{B}} \mathrm{T}$ of the mobility gap. However, since the activation energies we obtained were for $\mathrm{E}_{\mathrm{ex}}>2.8 \mathrm{eV}$, we are already mainly exciting the non-localized states; it is thus unclear as to why emission intensity would be seriously affected by the presence of the additional carriers made possible by thermal excitation above the mobility gap.

One possibility is that there are several activation energies, and that our selectively excited temperature dependence experiments may have probed the lower energy ones, while other experiments which have used higher temperatures have been able to probe the higher activation energies. This view is supported by noting that the quenching we observed between $12 \mathrm{~K}$ and $300 \mathrm{~K}$, roughly a factor of 10 , is about the same as the quenching observed by groups who measured a much higher activation energy (as mentioned in section 3.3), despite the fact that our thermal quenching began at $80-90 \mathrm{~K}$ as opposed to their $250 \mathrm{~K}$ [76].

It would also have been interesting to measure activation energies for $\mathrm{E}_{\mathrm{ex}}<2.8 \mathrm{eV}$ (below the mobility gap), to compare with our reported values for $\mathrm{E}_{\mathrm{ex}}>2.8 \mathrm{eV}$, since a difference would be expected in that case. However, the low signal produced by low excitation energies (due to low absorption), and the low signal seen at higher temperatures (due to thermal quenching) combined to make such measurements impossible.

Other, more "exotic" theories may also be invoked to explain the difference in activation energies between our measurements and those of other groups. One such possibility would be if in the case of above 
band-gap excitation, the dominant non-radiative recombination centers are saturated. In that case, we would be measuring the activation energy of exciting the carriers from the defects involved in emission, and the larger activation energies measured by other groups might be the energy required to thermally empty the saturated non-radiative centers.

\subsection{Conclusion}

We have interpreted the results of our selective excitation of the BL in Mg-doped GaN in a model of DAP recombination in the presence of large potential fluctuations, together with a large amount of disorder. The Mg-doped GaN sample we have studied displays certain similarities to amorphous and other highly disordered materials. It exhibits an Urbach tail, having an Urbach parameter of $E_{0}=33 \mathrm{meV}$. We propose that a mobility gap exists at $2.8 \mathrm{eV}$, whereby carriers in defects with energy higher than this gap are able to transfer to other defects, and carriers in defects with energy lower than this gap are strongly localized. The constant peak shift between excitation and emission for $\mathrm{E}_{\mathrm{ex}}<2.8 \mathrm{eV}(180 \mathrm{meV})$ was interpreted as a Frank-Condon shift resulting from strong electron-LO phonon interaction. This coupling is responsible for the large width of the BL even after removal of the inhomogeneous broadening by the selective excitation process. The linear dependence of the peak position on $E_{e x}$ for $E_{e x}>2.8 \mathrm{eV}$ was attributed to either screening or band-filling effects related to the larger number of free carriers present at higher excitation energies. The low activation energies from temperature dependent experiments may be a result of an inability to reach high enough temperatures to probe the activation energies reported by other groups. 


\section{Figure Captions for Chapter 3}

Fig. 3-1 Blue luminescence of our Mg-doped GaN sample, excited as normal (above the band-gap) photoluminescence by a $\mathrm{HeCd}$ laser at $12 \mathrm{~K}$.

Fig. 3-2 $12 \mathrm{~K}$ normal BL (solid line as in Fig. 3-1, excited at 3.815 eV) compared with a representative $12 \mathrm{~K}$ selectively excited BL (broken line, excited at $2.878 \mathrm{eV}$ ).

Fig. 3-3 Representative $12 \mathrm{~K}$ selectively excited BL, excited by dye laser energies between $2.687 \mathrm{eV}$ (lower curve) and $2.999 \mathrm{eV}$ (upper curve). The PL is plotted as a function of the difference between emission and excitation energies.

Fig. 3-4 Summary of $12 \mathrm{~K}$ selectively excited BL peak positions (the shift from the excitation energy), plotted as a function of excitation energy. The excitation laser energy was varied from 2.687 to $2.999 \mathrm{eV}$. The dashed line is a linear fit to data above $2.8 \mathrm{eV}$. The horizontal solid line and the sloped dotted line are what would be expected for normal Raman and PL behavior, and have been labeled accordingly.

Fig. 3-5 Summary of the integrated intensity of the $12 \mathrm{~K}$ selectively excited BL peaks. The excitation laser was varied from 2.687 to $2.999 \mathrm{eV}$. The solid line is a linear least-squares fit of the log integrated intensity to the excitation energy. The result of the fit shows $\mathrm{I} \sim \exp \left(E_{e x} / E_{0}\right)$ with $E_{0}=33 \mathrm{meV}$.

Fig. 3-6 The selectively excited PL (excited at $2.887 \mathrm{eV}$ ), for various temperatures between $12 \mathrm{~K}$ and $299 \mathrm{~K}$. The PL is plotted on a $\log _{10}$ scale as a function of the difference between the emission and excitation energies.

Fig. 3-7 Arrhenius plot for the temperature dependent PL shown in Fig. 3-6 (with $\mathrm{E}_{\mathrm{ex}}=2.887 \mathrm{eV}$ ). The activation energy obtained by fitting the temperature dependence of the selectively excited BL to Equation 
$(2-1)$ is $22 \mathrm{meV}$. The same activation energy $( \pm 3 \mathrm{meV})$ is obtained for all excitation energies between 2.8 $\mathrm{eV}$ and $3.0 \mathrm{eV}$.

Fig. 3-8 Schematic picture of the model explaining the selectively-excited BL results, incorporating a mobility gap at $2.8 \mathrm{eV}$. If DAPs with energies above the mobility gap are excited, a transfer of energy to other DAP states (with lower energies) may occur. If DAPs are excited below the mobility gap, recombination at the same DAP (observed with a Frank-Condon shift) will occur. The horizontal lines depict in a rough way the available states. The exponential density of states as a function of energy is plotted on the right hand side. 


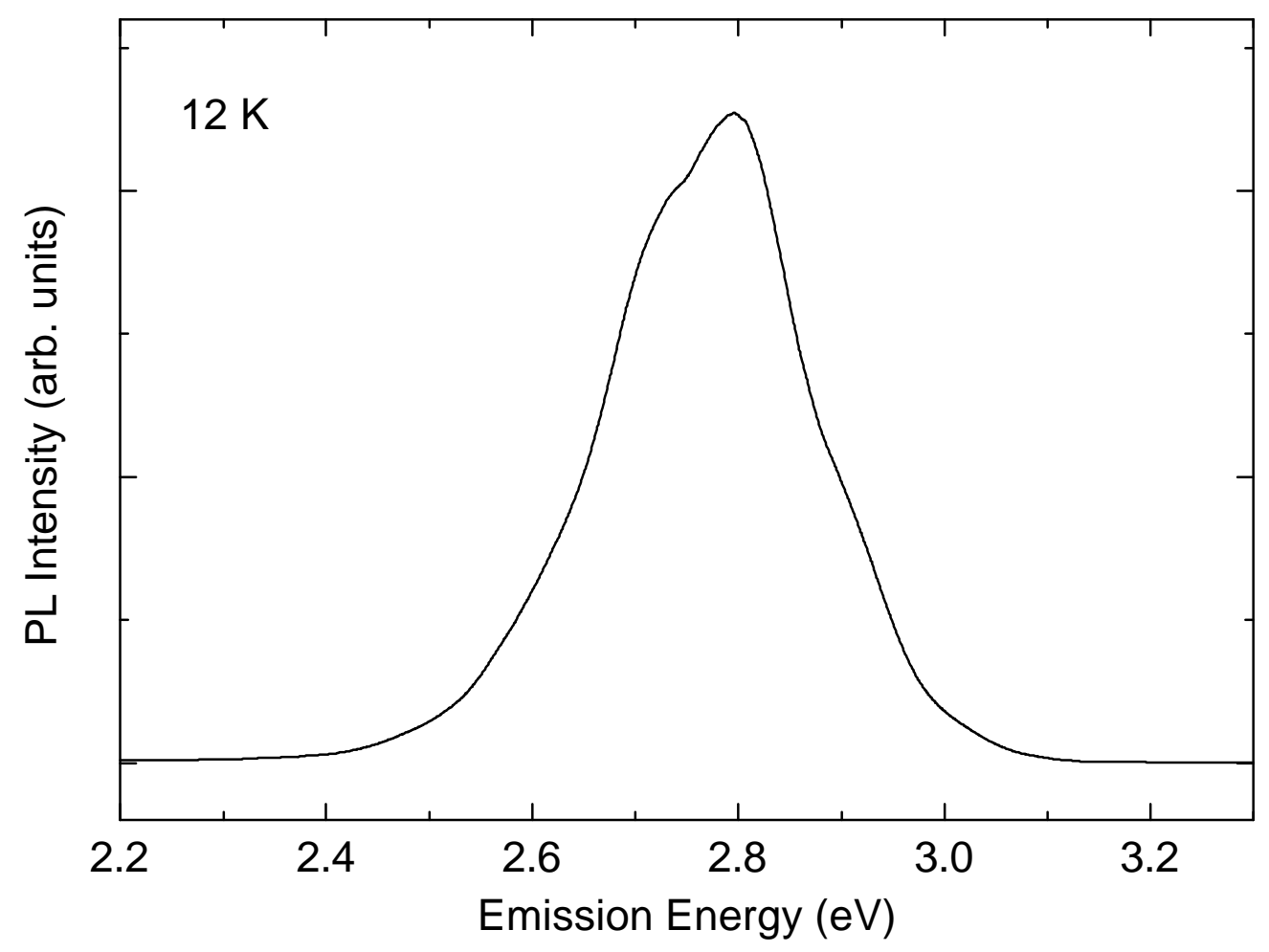

Figure 3-1 


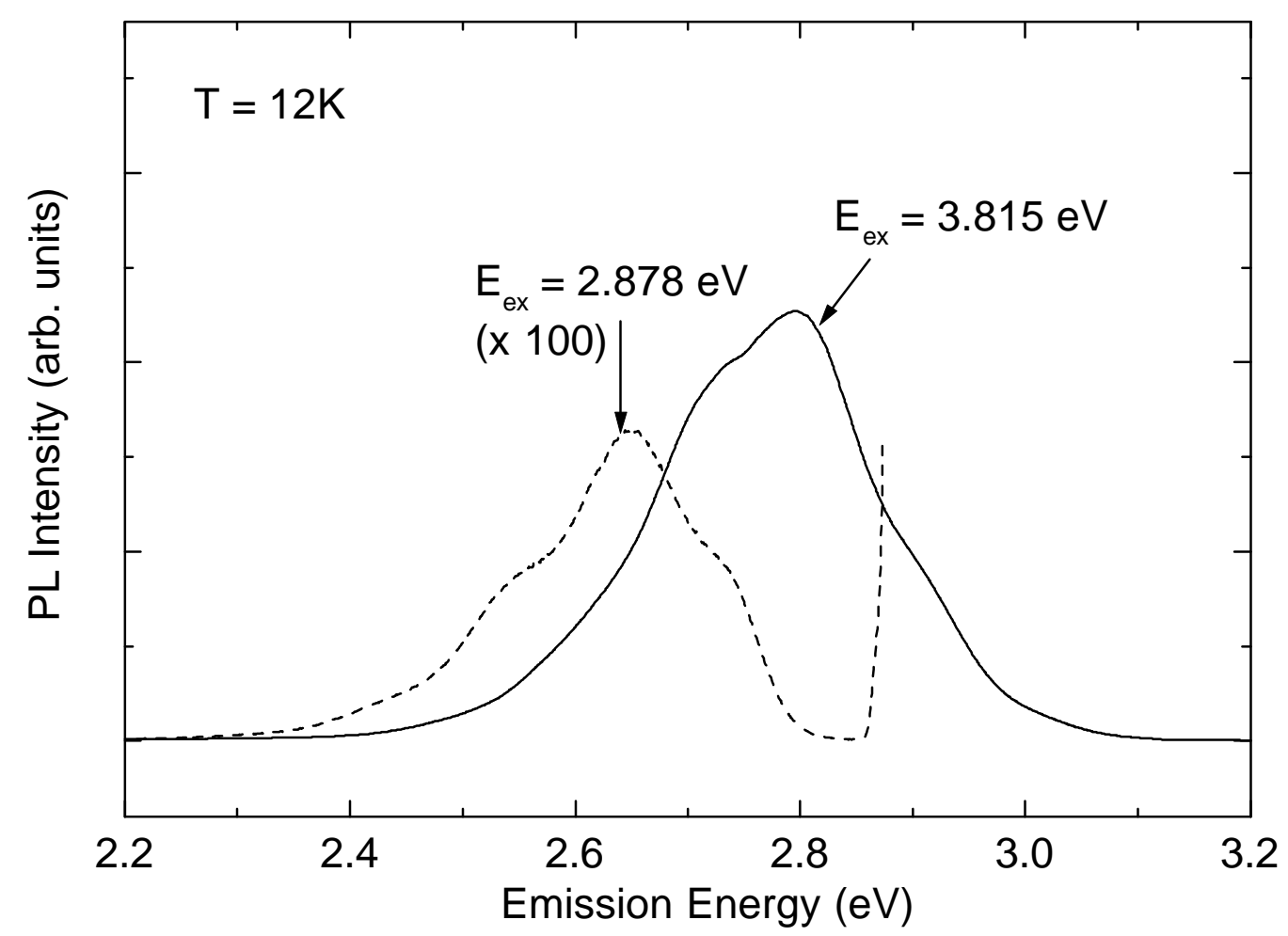

Figure 3-2 


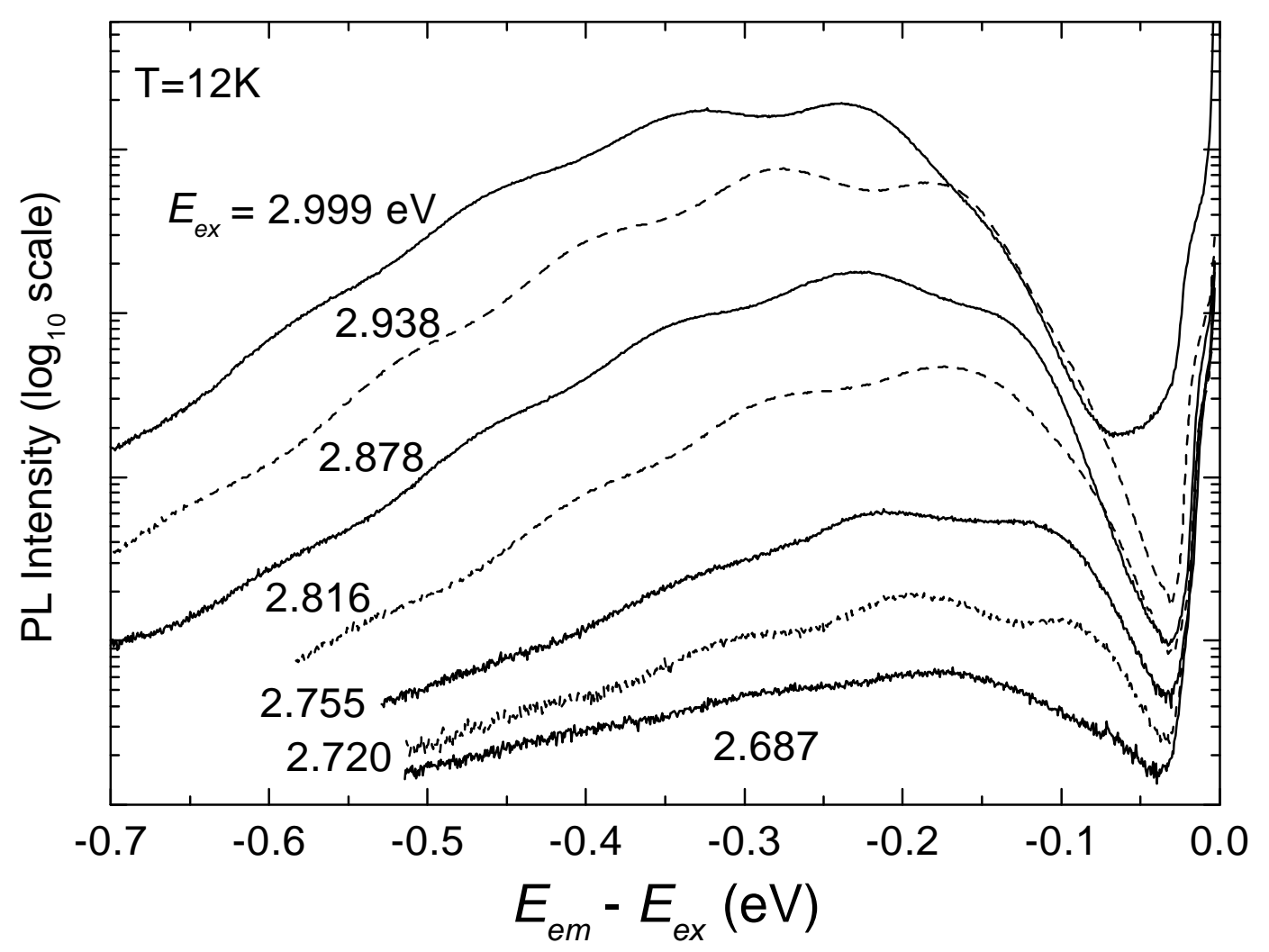

Figure 3-3 


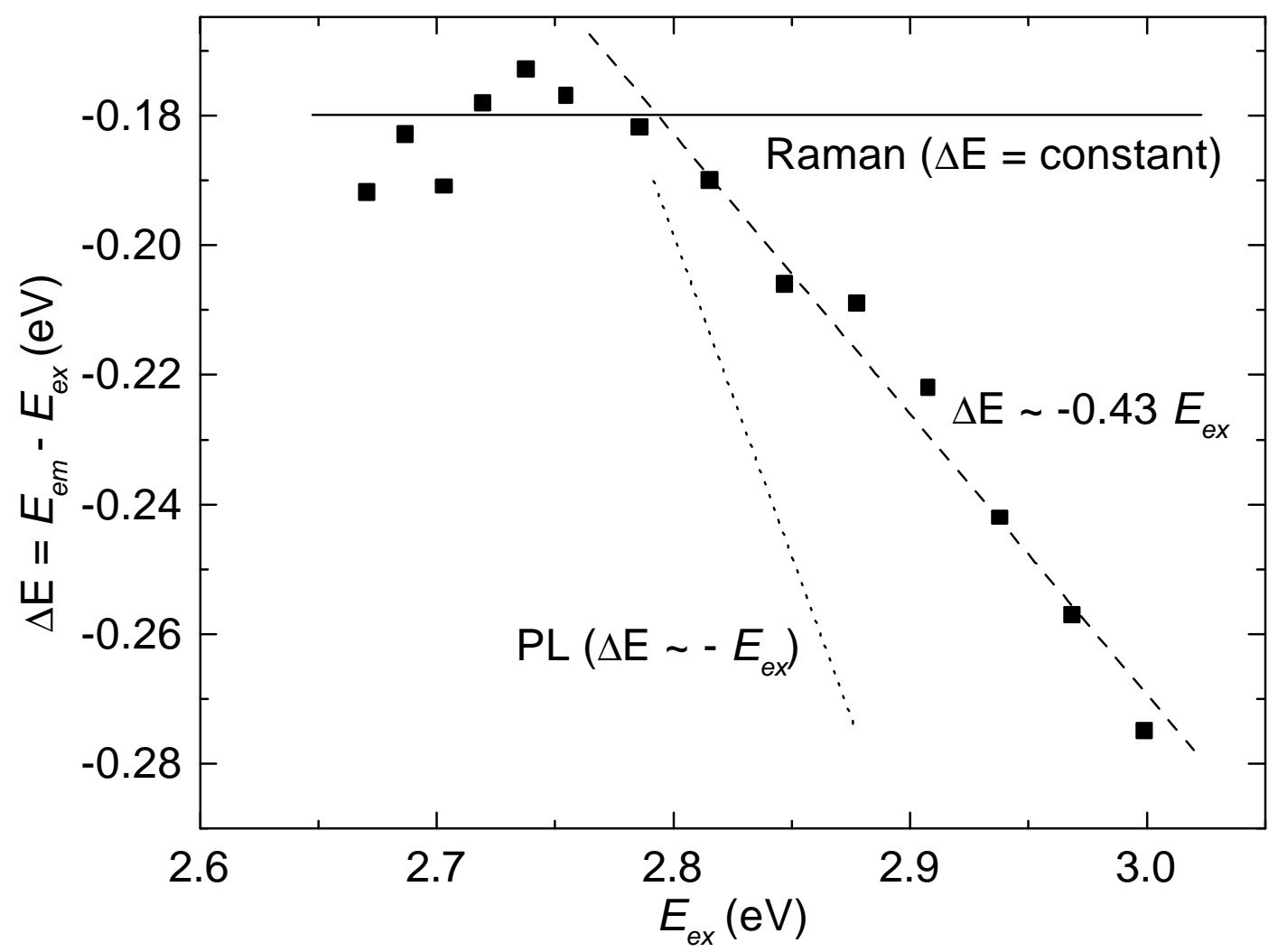

Figure 3-4 


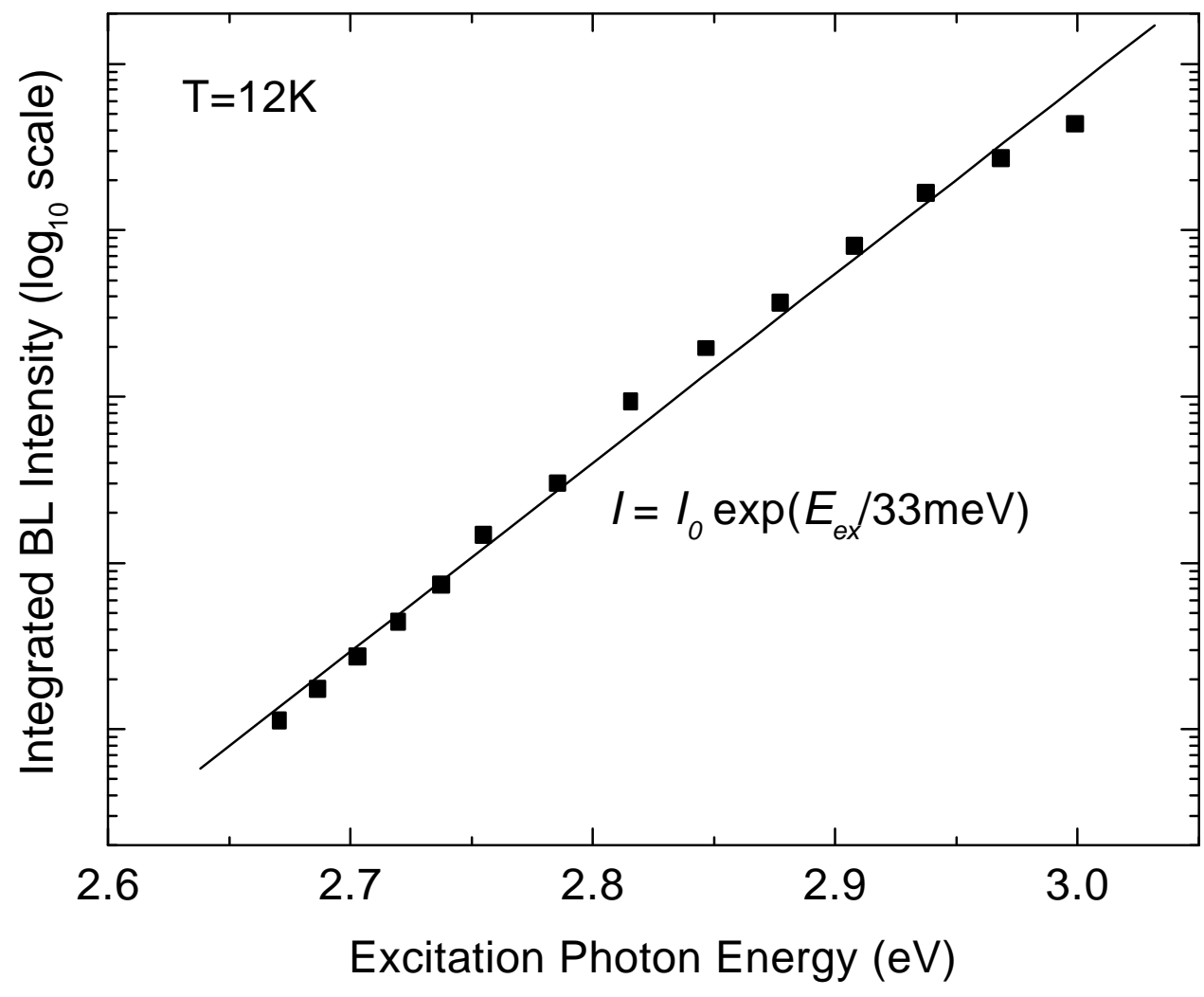

Figure 3-5 


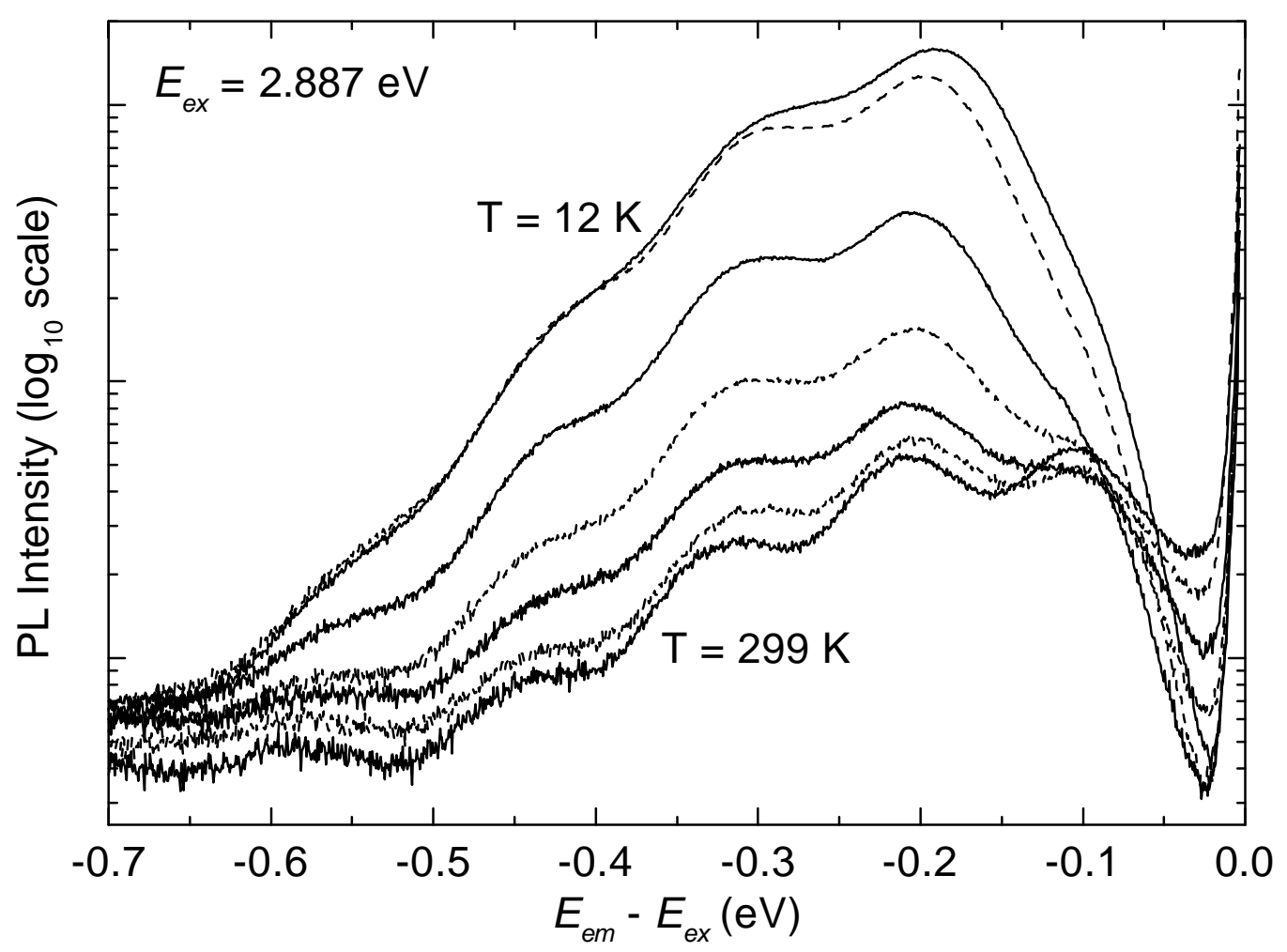

Figure 3-6 


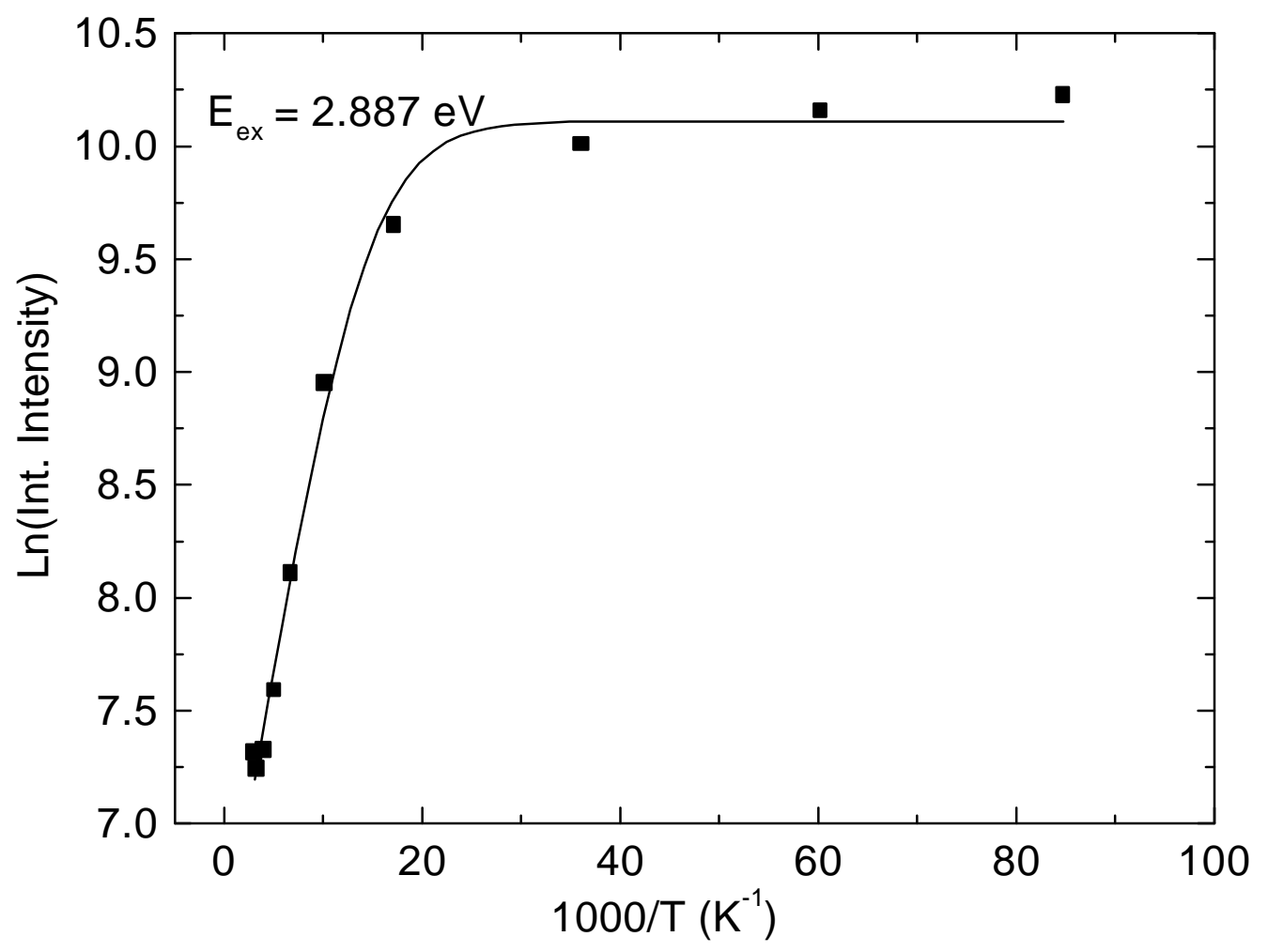

Figure 3-7 


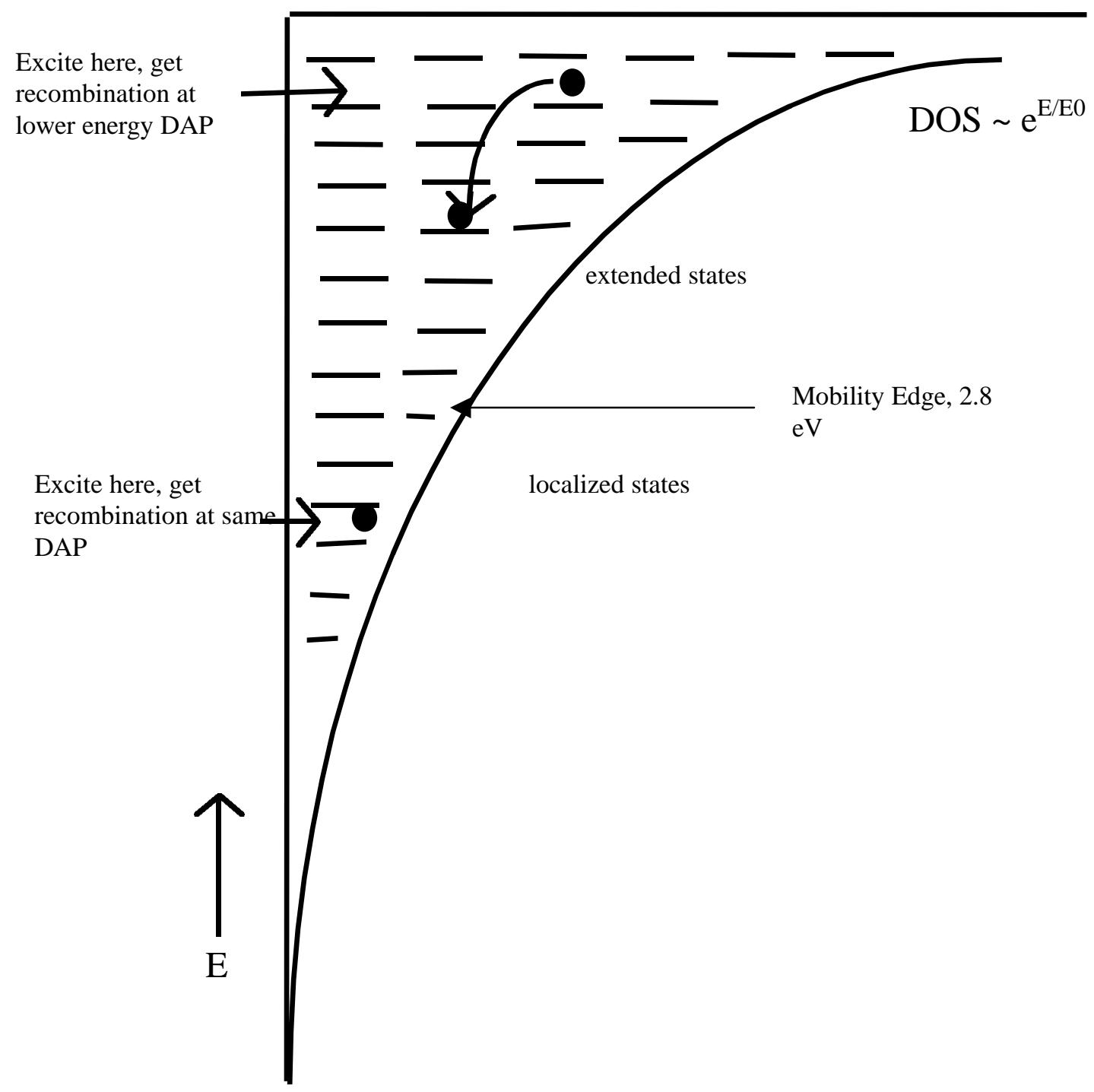

Figure 3-8 


\section{Chapter 4: Conclusions}

$\mathrm{GaN}$ is an interesting material: technologically very useful, but still having many unexplained features. Two such features are the broad defect-related luminescence bands: the YL of $n$-type GaN and the $\mathrm{BL}$ of Mg-doped $p$-type GaN. We have employed selective excitation to investigate these bands.

In the case of the YL, most of the previous evidence has supported a recombination model between distant donors and acceptors, most likely a transition involving a shallow donor to a deep acceptor. Our selective excitation experiments have resolved finer structures within the YL. Our results indicate that the YL in bulk samples is related to the YL in film samples. We suggest that selectively excited YL involves recombination at DAP complexes, rather than between spatially distant DAPs (however other recombination channels, including that of distant DAPs may become significant under other excitation conditions). Characteristics of the DAP complexes within our YL model include (a) an electron localization energy of around 60-70 meV, (b) a localized phonon energy of around $40 \mathrm{meV}$, and (c) excited states of the complex at 200 and $370 \mathrm{meV}$ above the ground state.

In the case of the BL, the deep defect responsible for the BL is unknown, and there may not even be a deep defect involved. Also in dispute is the role of potential fluctuations in the properties of the BL. Our results have been explain in a model whereby emission is from DAPs, and significant effects are produced by doping-related potential fluctuations and disorder. Characteristics of the our model for the BL include (a) an Urbach tail, having width $\mathrm{E}_{0}=33 \mathrm{meV}$, (b) a strong electron-LO phonon coupling occurring with a Frank-Condon shift of $\sim 180 \mathrm{meV}$ between excitation and emission, (c) a mobility gap at $2.8 \mathrm{eV}$, separating highly mobile states and highly localized states, and (d) PL-like behavior for excitation energies larger than $2.8 \mathrm{eV}$, having a blue-shift with increasing excitation energy caused by the increased number of free carriers in the material. 


\section{References}

[1] M. Leszczynski, H. Teisseyre, T. Suski, I. Grzegory, M. Bockowski, J. Jun, S. Porowski, K. Pakula, J. M. Baranowski, C. T. Foxon, and T. S. Cheng, Appl. Phys. Lett. 69, 73 (1996).

[2] I. H. Oguzman, J. Kolnik, K. F. Brennan, R. Wang, and P. P. Ruden, Mat. Res. Soc. Symp. Proc. 395, 479 (1996).

[3] Phonon dispersion reference

[4] S. Nakamura and G. Fasol, The Blue Laser Diode, Springer-Verlag, New York, 1997.

[5] I. Akasaki, Mat. Res. Soc. Symp. Proc. 482, 3 (1998).

[6] B. Monemar, J. Cryst. Growth 189/190, 1 (1998).

[7] S. Strite and H. Morkoç, J. Vac. Sci. Technol. B 10, 1237 (1992).

[8] W. C. Johnson, J. B. Parsons, and M. C. Crew, J. Phys. Chem. 36, 2651 (1932).

[9] H. P. Maruska and J. J. Tietjen, Appl. Phys. Lett. 15, 327 (1969).

[10] J. I. Pankove, E. A. Miller, and J. E. Berkeyheiser, RCA Review 32, 383 (1971).

[11] S. D. Lester, F. A. Ponce, M. G. Craford, and D. A. Steigerwald, Appl. Phys. Lett. 66, 1249 (1995).

[12] H. Amano, N. Sawaki, I. Akasaki, and Y. Toyoda, Appl. Phys. Lett. 48, 353 (1986).

[13] S. Nakamura, Jpn. J. Appl. Phys. 30, L 1705 (1991).

[14] M. E. Lin, S. Strite, A. Agarwal, A. Salvador, G. L. Zhuo, N. Teraguchi, A. Rockett, and H. Morkoç, Appl. Phys. Lett. 62, 702 (1993).

[15] A. Kuramata, K. Horino, K. Domen, K. Shinohara, and T. Tanahashi, Appl. Phys. Lett. 67, 2521 (1995).

[16] T. S. Zheleva, O.-H. Nam, M. D. Bremser, and R. F. Davis, Appl. Phys. Lett. 71, 2472 (1997).

[17] T. Suski, P. Perlin, H. Teisseyre, M. Leszczynski, I. Grzegory, J. Jun, and S. Porowski, Proc. $22^{\text {nd }}$ Inter. Conf. Phys. Semicond., vol. 1, pg. 397.

[18] H. Amano, M. Kitoh, K. Hiramatsu, N. Sawaki, and I. Akasaki, Jpn. J. Appl. Phys. 28, L 2112 (1989).

[19] S. Nakamura, T. Mukai, M. Senoh, and N. Iwasa, Jpn. J. Appl. Phys. 31, L 139 (1992).

[20] S. Nakamura, N. Iwasa M. Senoh, and T. Mukai, Jpn. J. Appl. Phys., Part 1, 31, 1258 (1992). 
[21] S. Nakamura, T. Mukai, and M. Senoh, Appl. Phys. Lett. 64, 1687 (1994).

[22] S. Nakamura, M. Senoh, S. Nagahama, N. Iwasa, T. Yamada, T. Matzushita, H. Kiyoku, and Y. Sugimoto, Jpn. J. Appl. Phys. 35, L 74 (1996).

[23] M. Ilegems and H. C. Montgomery, J. Phys. Chem. Solids 34, 885 (1973).

[24] P. Perlin, T. Suski, H. Teisseyre, M. Leszczynski, I. Grzegory, J. Jun, S. Porowski, P. Boguslawski, J. Bernholc, J. C. Chervin, A. Polian, and T. D. Moustakas, Phys. Rev. Lett. 75, 296 (1995).

[25] P. Boguslawski, E. L. Briggs, and J. Bernholc, Phys. Rev. B 51, 17255 (1995).

[26] W. Götz, N. M. Johnson, C. Chen, H. Liu, C. Kuo, and W. Imler, Appl. Phys. Lett. 68, 3144 (1996).

[27] C. Wetzel, T. Suski, J. W. Ager III, E. R. Weber, E. E. Haller, S. Fischer, B.K. Meyer, R. J. Molnar, and P. Perlin, Phys. Rev. Lett. 78, 3923 (1997).

[28] C. G. Van de Walle, C. Stampfl, and J. Neugebauer, J. Cryst. Growth 189/190, 505 (1998).

[29] T. Mattila and R. M. Nieminen, Phys. Rev. B 55, 9571 (1997).

[30] D. J. Chadi and K. J. Chang, Phys. Rev. Lett. 61, 873 (1988).

[31] M. Drechsler, D. M. Hofmann, B. K. Meyer, T. Detchprohm, H. Amano, and I. Akasaki, Jpn. J. Appl. Phys, Part 2, 34, L 1178 (1995).

[32] A. S. Barker, Jr., and M. Ilegems, Phys. Rev. B 7, 743 (1973).

[33] H. Wang and A.-B. Chen, J. Appl. Phys. 87, 7859 (2000).

[34] W. Götz, N. M. Johnson, J. Walker, D. P. Bour, and R. A. Street, Appl. Phys. Lett. 68, 667 (1996).

[35] M. Ilegems and R. Dingle, J. Appl. Phys. 44, 4234 (1973).

[36] F. Mireles and S. E. Ulloa, Phys. Rev. B 58, 3879 (1998).

[37] M. Suzuki, T. Uenoyama, and A. Yanase, Phys. Rev. B 52, 8132 (1995).

[38] C. Merz, M. Kunzer, U. Kaufmann, I. Akasaki, and H. Amano, Semicond. Sci. Technol. 11, 712 (1996).

[39] J. J. Pankove, S. Bloom, and G. Harbecke, RCA Rev 36, 163 (1975).

[40] J. S. Im, A. Moritz, F. Steuber, V. Härle, F. Scholz, and A. Hangleiter, Appl. Phys. Lett. 70, 631 (1997). 
[41] J. Neugebauer and C. G. Van de Walle, Phys. Rev. B 50, 8067 (1994).

[42] J.I. Pankove, and J.A. Hutchby, J. Appl. Phys. 47, 5387 (1976).

[43] T. Ogino and M. Aoki, Jap. J. Appl. Phys. 19, 2395 (1980).

[44] P. Y. Yu and M. Cardona, Fundamentals of Semiconductors, Springer-Verlag, New York, 1996, pg. 344.

[45] E. R. Glaser, T. A. Kennedy, K. Doverspike, L. B. Rowland, D. K. Gaskill, J. A. Freitas, Jr., M. Asif Khan, D. T. Olson, J. N. Kuznia, and D. K. Wickenden, Phys. Rev. B 51, 13326 (1995).

[46] P. W. Mason, A. Dörnen, V. Haerle, F. Scholz, G. D. Watkins, Mat. Res. Soc. Symp. Proc. 449, 793 (1997).

[47] T. L. Tansley and R. J. Egan, Phys. Rev. B 45, 10942 (1992).

[48] D. W. Jenkins and J. D. Dow, Phys. Rev. B 39, 3317 (1989).

[49] J. I. Pankove, H. P. Maruska, and J. E. Berkeyheiser, Appl. Phys. Lett. 17, 197 (1970).

[50] D.M. Hofmann, D. Kovalev, G. Steude, B.K. Meyer, A. Hoffmann, L. Eckey, R. Heitz, T. Detchprom, H. Amano, and I. Akasaki, Phys. Rev. B 52, 16702 (1995).

[51] Yu and Cardona, pg. 348.

[52] D. G. Thomas, J. J. Hopfield, and W. M. Augustyniak, Phys. Rev. 140, A202 (1965).

[53] T. Suski, P. Perlin, H. Teisseyre, M. Leszczynski, I. Grzegory, J. Jun, M. Bockowski, S. Porowski, and T.D. Moustakes, Appl. Phys. Lett. 67, 2188 (1995).

[54] J. Neugebauer and C. G. Van de Walle, Appl. Phys. Lett. 69, 503 (1996).

[55] F.A. Ponce, D.P. Bour, W. Götz, and J.P. Wright, Appl. Phys. Lett. 68, 57 (1996).

[56] X. Zhang, P. Kung, and M. Razeghi, Mat. Res. Soc. Symp. Proc. 395, 625 (1996).

[57] K. Saarinen, T. Laine, S. Kuisma, J. Nissilä, P. Hautojärvi, L. Dobrzynski, J.M. Baranowski, K. Pakula, R. Stephniewski, M. Wojdak, A. Wysmolek, T. Suski, M. Leszcynski, I. Grzegory, and S. Porowski, Phys. Rev. Lett. 79, 3030 (1997).

[58] E. Calleja, F.J. Sánchez, D. Basak, M.A. Sánchez-García, E. Muñoz, I. Izpura, F. Calle, J.M.G. Tijero, J.L. Sánchez-Rojas, B. Beaumont, P. Lorenzini, and P. Gibart, Phys. Rev. B 55, 4689 (1997). 
[59] I.-H. Lee, I.-H. Choi, C.R. Lee, and S.K. Noh, Appl. Phys. Lett. 71, 1359 (1997).

[60] G.-C. Yi and B. W. Wessels, Appl. Phys. Lett. 69, 3028 (1996).

[61] H.M. Chen, Y.F. Chen, M.C. Lee, and M.S. Feng, Phys. Rev. B 56, 6942 (1997).

[62] C.V. Reddy, K. Balakrishnan, H. Okumura, and S. Yoshida, Appl. Phys. Lett. 73, 244 (1998).

[63] U. Kaufmann, M. Kunzer, H. Obloh, M. Maier, C. Manz, A. Ramakrishnan, and B. Santic, Phys. Rev. B 59, 5561 (1999).

[64] I. Shalish, L. Kronik, G. Segal, Y. Rosenwaks, Y. Shapira, U. Tisch, and J. Salzman, Phys. Rev. B 59, 9748 (1999).

[65] E. R. Glaser, Private communication (2000).

[66] G. Li, S.J. Chua, S.J. Xu, W. Wang, P. Li, B. Beaumont, and P. Gibart, Appl. Phys. Lett. 74, 2821 (1999).

[67] W. Shan, T. J. Schmidt, R. J. Hauenstein, J. J. Song, and B. Goldenberg, Appl. Phys. Lett. 66, 3492 (1995).

[68] R. Zhang and T.F. Kuech, Appl. Phys. Lett. 72, 1611 (1998).

[69] Y.-H. Kwon, S.K. Shee, G.H. Gainer, G.H. Park, S.J. Hwang, and J.J. Song, Appl. Phys. Lett. 76, $840(2000)$.

[70] H. Amano, M. Kitoh, K. Hiramatsu, and I. Akasaki, J. Electrochem. Soc. 137, 1639 (1990).

[71] A.K. Viswanath, E. Shin, J.I. Lee, S. Yu, D. Kim, B. Kim, Y. Choi, and C.-H. Hong, J. Appl. Phys. 83, 2272 (1998).

[72] M. Smith, G.D. Chen, J.Y. Lin, H.X. Jiang, A. Salvador, B.N. Sverdlov, A. Botchkarev, H. Morkoc, and B. Goldenberg, Appl. Phys. Lett. 68, 1883 (1996).

[73] J.M. Myoung, K.H. Shim, C. Kim, O. Gluschenkov, K. Kim, S. Kim, D.A. Turnbull, and S.G. Bishop, Appl. Phys. Lett. 69, 2722 (1996).

[74] E. Oh, H. Park, and Y. Park, Appl. Phys. Lett. 72, 70 (1998).

[75] U. Kaufmann, M. Kunzer, M. Maier, H. Obloh, A. Ramakrishnan, B. Santic, and P. Schlotter, Appl. Phys. Lett. 72, 1326 (1998).

[76] M.A. Reshchikov, G.C. Yi, and B.W. Wessels, Phys. Rev. B 59, 13176 (1999). 
[77] W. Götz, N.M. Johnson, J. Walker, D.P. Bour, and R.A. Street, Appl. Phys. Lett. 68, 667 (1996).

[78] L. Eckey, U. von Gfug, J. Holst, A. Hoffmann, A. Kaschner, H. Siegle, C. Thomsen, B. Schineller, K. Heime, M. Heuken, O. Schön, and R. Beccard, J. Appl. Phys. 84, 5828 (1998).

[79] B. I. Shklovskii and A. L. Efros, Electronic Properties of Doped Semiconductors, Sprnger-Verlag, New York, 1984, pp. 65-73.

[80] Shklovskii and Efros, pp. 297-299.

[81] P. Bäume, J. Gutowski, D. Wiesmann, R. Heitz, A. Hoffmann, E. Kurtz, D. Hommel, and G. Landwehr, Appl. Phys. Lett. 67, 1914 (1995).

[82] P.W. Yu, J. Appl. Phys. 48, 5043 (1977).

[83] C. Kothandaraman, G.F. Neumark, and R.M. Park, Appl. Phys. Lett. 67, 3307 (1995).

[84] J.W. Allen, Semicond. Sci. Technol. 10, 1049 (1995).

[85] D. K. Watts, Point Defects in Crystals, John Wiley \& Sons, New York, 1977, pg. 252.

[86] J. Neugebauer and C. G. Van de Walle, Mat. Res. Soc. Symp. Proc. 395, 645 (1996).

[87] H. Obloh, K. H. Bachem, U. Kaufmann, M. Kunzer, M. Maier, A. Ramakrishnan, and P. Schlotter, J. Cryst. Growth 195, 270 (1998).

[88] P. Hacke, H. Nakayama, T. Detchprohm, K. Hiramatsu, and N. Sawaki, Appl. Phys. Lett. 68, 1362 (1996).

[89] R. Seitz, C. Gaspar, T. Monteiro, E. Pereira, et al., Mat. Sci. Forum 258-263, 1155 (1997).

[90] U. Kaufmann, M. Kunzer, H. Obloh, M. Maier, C. Manz, A. Ramakrishnan, and B. Santic, Phys. Rev. B 59, 5561 (1999).

[91] C. H. Park and D. J. Chadi, Phys. Rev. B 55, 12995 (1997).

[92] S.G. Lee and K.J.Chang, Semicond. Sci. Technol. 14, 138 (1999).

[93] F. Shahedipour and B.W. Wessels, Appl. Phys. Lett. 76, 3011 (2000).

[94] T. Suski, Private communication (2000).

[95] E. Glaser, Private communication (2000).

[96] J.K. Sheu, Y.K. Su, G.C. Chi, B.J. Pong, C.Y. Chen, C.N. Huang, and W.C. Chen, J. Appl. Phys. 84, 4590 (1998). 
[97] J. H. Edgar, Properties of Group III Nitrides, ed. by James H. Edgar, EMIS Datareview Series No. 11, INSPEC, the Institution of Electrical Engineers, London, 1994, pg. 3.

[98] J. S. Colton, P. Y. Yu, K. L. Teo, P. Perlin, E. R. Weber, I. Grzegory, and K. Uchida, Physica B 273-274, 75 (1999), and in Colton, et al., Appl. Phys. Lett. 75, 3273 (1999).

[99] H. Tews, H. Venghaus, and P. J. Dean, Phys. Rev. B 23, 4097 (1981).

[100] P. Y. Yu and C. Hermann, Phys. Rev. B 23, 4097 (1981).

[101] M. Rosenbauer, S. Finkbeiner, E. Bustarret, J. Weber, and M. Stutzmann, Phys. Rev. B 51, 10539 (1995).

[102] A. Saito and T. Suemoto, Phys. Rev. B 23, 4097 (1997).

[103] N. Weiser, O. Ambacher, H.-P. Felsl, L. Gögens, and M. Stutzmann, Appl. Phys. Lett. 74, 3981 (1999).

[104] S. Porowski and I. Grzegory, in GaN and Related Materials, edited by J. Pearton, Optoelectronic Properties of Semiconductors and Superlattices vol. 2, Gordon and Breach, Amsterdam, p. 295.

[105] Shklovskii and Efros, pg. 51.

[106] H. C. Casey, Jr., F. Ermanis, and k. B. Wolfstirn, J. Appl. Phys. 40, 2945 (1969).

[107] K. Pakula, M. Wojdak, M. Palczewska, B. Suchanek, and J. M. Baranowski, MRS Internet J. Nitride Semicond. Res. 3, 34 (1998).

[108] A. S. Barker, Jr., and A. J. Sievers, Rev. Mod. Phys. 47, Suppl. 2, S1 (1975).

[109] J. Pankove, Optical Processes in Semiconductors, Prentice-Hall Inc., New Jersey, 1971, pg. 43.

[110] K. W. Böer, Survey of Semiconductor Physics, Van Nostrand Reinhold, New York, 1990, pg. 633

[111] T. W. Kang, Sh. U. Yuldashev, I. L. Bolotin, S. H. Park, D. Y. Kim, S. H. Won, K. S. Jung, and T. W. Kim, J. Appl. Phys. 88, 790 (2000).

[112] C. H. Qiu, C. Hoggatt, W. Melton, M. W. Leksono, and J. I. Pankove, Appl. Phys. Lett. 66, 2712 (1995).

[113] S. Chichibu, T. Mitzutani, T. Shioda, H. Nakanishi, T. Deguchi, T. Azuhata, T. Sota, and S. Nakamura, Appl. Phys. Lett. 70, 3440 (1997).

[114] Böer, pg. 515. 
[115] Yu and Cardona, pg. 381.

[116] Böer, pg. 910.

[117] T. Tiedje, Semiconductors and Semimetals 21 C, 207 (1984).

[118] Böer, pg. 918.

[119] I. Kuskovsky, D. Li, G.F. Neumark, V.N. Bondarev, and P.V. Pikhitsa, Appl. Phys. Lett. 75, 1243 (1999). 